\title{
AMOSTRAGEM SEQÜENCIAL (PRESENÇA-AUSÊNCIA) PARA O MONITORAMENTO DA COCHONILHA-BRANCA AULACASPIS TUBERCULARIS (NEWSTEAD) (HEMIPTERA: DIASPIDIDAE) NA CULTURA DA MANGA
}

WILLIAN VICTOR SACONATO

Dissertação apresentada à Escola Superior de Agricultura "Luiz de Queiroz", Universidade de São Paulo, para obtenção do título de Mestre em Agronomia, Área de Concentração: Estatística e Experimentação Agronômica.

P I R A C I C A B A

Estado de São Paulo - Brasil

Julho - 2005 


\section{AMOSTRAGEM SEQÜENCIAL (PRESENÇA-AUSÊNCIA) PARA O MONITORAMENTO DA COCHONILHA-BRANCA $A U L A C A S P I S T U B E R C U L A R I S$ (NEWSTEAD) (HEMIPTERA: DIASPIDIDAE) NA CULTURA DA MANGA}

\section{WILLIAN VICTOR SACONATO}

Licenciado em Matemática

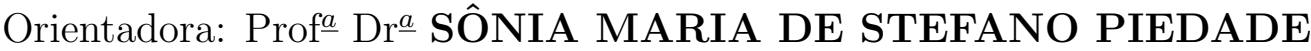

Dissertação apresentada à Escola Superior de Agricultura "Luiz de Queiroz", Universidade de São Paulo, para obtenção do título de Mestre em Agronomia, Área de Concentração: Estatística e Experimentação Agronômica.

P I R A C I C A B A

Estado de São Paulo - Brasil

Julho - 2005 
Dados Internacionais de Catalogação na Publicação (CIP) DIVISÃO DE BIBLIOTECA E DOCUMENTAÇÃO - ESALQ/USP

Saconato, Willian Victor

Amostragem seqüencial (presença-ausência) para o monitoramento da cochonilha-

branca Aulacaspis tubercularis (Newstead) (Hemíptera: Diaspididae) na cultura da manga I Willian Victor Saconato. - - Piracicaba, 2005.

$130 \mathrm{p}$.

Dissertação (Mestrado) - - Escola Superior de Agricultura Luiz de Queiroz, 2005. Bibliografia.

1. Amostragem seqüencial 2. Cochonilha-branca 3. Distribuição binomial 4. Manejo integrado 5. Manga 6. Monitoramento 7. População animal 8. Praga de planta I. Título

CDD 634.441

"Permitida a cópia total ou parcial deste documento, desde que citada a fonte - O autor" 


\title{
DEDICATÓRIA
}

Aos meus pais,

\section{Edson e Gelci}

pelo amor incondicional que foi fundamental nesta conquista, pelo apoio, dedicação e confiança, que nortearam minha vida e me fizeram progredir.

\author{
À minha irmã, cunhado e sobrinhos. \\ Niusley, Fernando, Bruno e Fernanda \\ a amizade e confiança. \\ Aos meus avós \\ Juvenal (in memorian) e Guerino (in memo- \\ rian) \\ e minhas avós.
}

\section{Maria Aparecida e Ivete}

pelo amor e carinho de sempre.

À minha esposa,

\section{Vanessa}

pelo companheirismo, amor, compreensão e apoio nos momentos decisivos. 


\section{AGRADECIMENTOS}

À Prof $\underline{a}^{\underline{a}} \operatorname{Dr}^{\underline{a}}$ Sônia Maria De Stefano Piedade a orientação e amizade, que foram fundamentais para a realização desse trabalho.

Ao Prof ${ }^{o}$ Dro Décio Barbin a amizade, ensinamentos e apoio.

Ao Miguel Francisco de Souza Filho do Instituto Biológico de Campinas pelo apoio e dados cedidos para a realização desse trabalho.

Aos meus pais Edson Lima Saconato e Gelci Dionete Bortoletto Saconato e minha esposa Vanessa Cristina Ferreira Saconato a confiança, o amor e por acreditarem e me fazer acreditar que chegar até aqui seria possível.

Aos amigos do mestrado e doutorado, em especial à Melissa Oda e Alfredo Venzel, e aos amigos David, Denise, Francine, Hélio, Michel e Gisele o apoio e a compreensão.

À Solange Sabadin e Luciane Brajão do Departamento de Ciências Exatas ESALQ/USP a paciência e atenção.

Aos engenheiros agrônomos do Comitê Gestor da PIF Manga Jair Marconato, João Murakami, Luis Bassoli, Mario Frederico, Ryosuke Kavati e Vera Palla pela orientação a respeito do monitoramento da cochonilha.

À bibliotecária Silvia Zinsly a revisão das normas.

Aos professores e funcionários do Departamento de Ciências Exatas da ESALQ/USP, que me propiciaram condições para a realização desse trabalho. 


\section{SUMÁRIO}

Página

LISTA DE FIGURAS . . . . . . . . . . . . . . . . . . . . vii

LISTA DE TABELAS . . . . . . . . . . . . . . . . . . ix

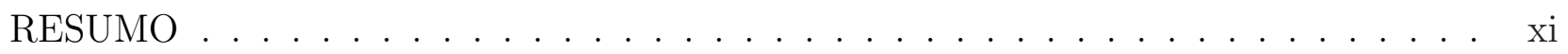

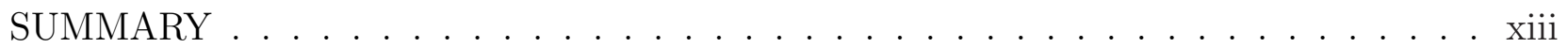

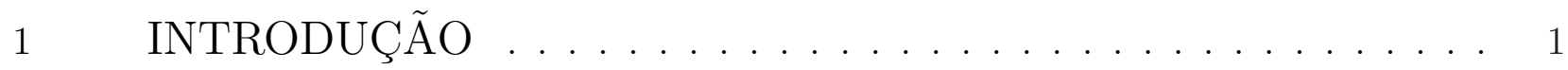

2 REVISÃO DE LITERATURA . . . . . . . . . . . . . . . . 4

$2.1 \quad$ Nível de Limiar Econômico . . . . . . . . . . . . . . . . . . . . . . . 8

2.2 Seleção de Níveis Máximos de Probabilidade . . . . . . . . . . . . . . . . 9

2.3 Erro de amostragem . . . . . . . . . . . . . . . 9

2.4 Tamanho da amostra e tempo de amostragem . . . . . . . . . . . . . 9

2.5 Teste seqüencial da razão de probabilidades . . . . . . . . . . . . . . . . . 11

2.6 Determinação dos valores $A$ e $B \ldots \ldots \ldots \ldots$

2.7 Probabilidade de aceitar $H_{0}$ ou $H_{1}$ quando alguma terceira hipótese $H$ é verdadeira 15

2.8 Número esperado de observações necessárias para se tomar uma decisão . . . 16

2.9 Testando uma determinada hipótese $H_{0}$ contra uma hipótese alternativa $H_{1}$. . 18

2.10 Plano de amostragem seqüencial para testar a média de uma distribuição binomial 19

2.10.1 Exposição do problema . . . . . . . . . . . . . . . . . . . . . . . . 19

2.10.2 Riscos tolerados de tomar decisões erradas . . . . . . . . . . . . . . . . . . . 19

2.10.3 Linhas de decisão do plano de amostragem seqüencial . . . . . . . . . . . . . 20

2.10.4 Curva característica operacional . . . . . . . . . . . . . . . 24

2.10.5 Curva do Número Médio de Unidades Amostrais . . . . . . . . . . . . . . . . 26

$3 \quad$ MATERIAL E MÉTODOS . . . . . . . . . . . . . . . 27

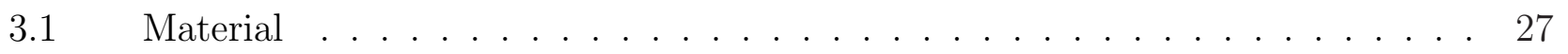

3.1.1 Localização e datas de amostragens . . . . . . . . . . . . . . . . . 27

$3.2 \quad$ Métodos . . . . . . . . . . . . . . . . . . 30 
3.2.1 Monitoramento . . . . . . . . . . . . . . . . . . . 30

3.2.2 Construção do plano de amostragem seqüencial . . . . . . . . . . . . . . 31

4 RESULTADOS E DISCUSSÃO . . . . . . . . . . . . . 33

4.1 Ajuste da distribuição binomial aos dados de presença-ausência . . . . . . . . . 33

$4.2 \quad$ Planos de amostragem seqüencial com níveis de erros iguais a $0,20 \ldots 36$

4.2.1 Plano de amostragem seqüencial para ramos no período vegetativo . . . . . . 36

4.2.2 Plano de amostragem seqüencial para ramos no período de frutificação . . . . . 39

4.2 .3 Plano de amostragem seqüencial para frutos . . . . . . . . . . . . . . . . . . 42

4.3 Planos de amostragem seqüencial com níveis de erros iguais a 0,10 . . . . . 45

4.3.1 Plano de amostragem seqüencial para ramos no período vegetativo . . . . . . 45

4.3.2 Plano de amostragem seqüencial para ramos no período de frutificação . . . . . 49

4.3.3 Plano de amostragem seqüencial para frutos . . . . . . . . . . . . . . 52

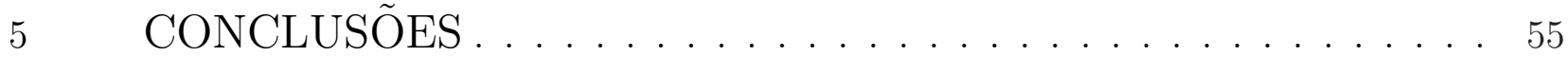

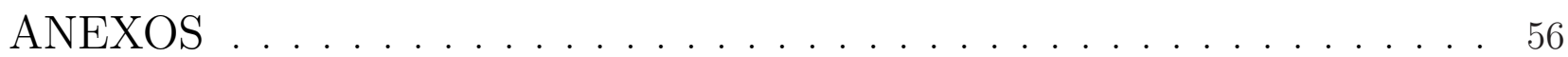

REFERÊNCIAS BIBLIOGRÁFICAS . . . . . . . . . . . . 125 


\section{LISTA DE FIGURAS}

Página

1 Linhas de decisão do plano de amostragem seqüencial com base na distribuição

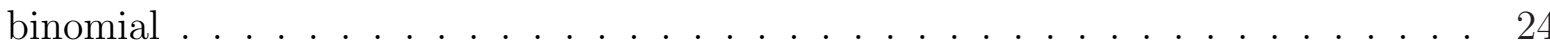

2 Curva característica operacional para o plano de amostragem seqüencial com base na distribuição binomial . . . . . . . . . . . . . . . . . . . 25

3 Curva do número médio de unidades amostrais para o plano de amostragem seqüencial com base na distribuição binomial . . . . . . . . . . . . . . . . . . . 26

4 Linhas de decisão do plano de amostragem seqüencial para ramos no período vegetativo, com níveis de erros iguais a 0,20, onde m é o número de plantas observadas e $\mathrm{km}$ é o número de plantas infestadas . . . . . . . . . . . . . . . . . . . 36

5 Curva característica operacional do plano de amostragem seqüencial para ramos no período vegetativo, com níveis de erros iguais a 0,20 , onde p é proporção de plantas infestadas e COp a probabilidade de aceitar $H_{0} \ldots \ldots \ldots$. . . . . . . . 38

6 Curva do número médio de unidades amostrais (Epn) do plano de amostragem seqüencial para ramos no período vegetativo, com níveis de erros iguais a 0,20, onde p é a proporção de plantas infestadas . . . . . . . . . . . . . . . . . . . . 39

7 Linhas de decisão do plano de amostragem seqüencial para ramos no período de frutificação, com níveis de erros iguais a 0,20 . . . . . . . . . . . . . . . 39

8 Curva característica operacional do plano de amostragem seqüencial para ramos no período de frutificação, com níveis de erros iguais a $0,20 \ldots \ldots$. . . . . .

9 Curva do número médio de unidades amostrais do plano de amostragem seqüencial para ramos no período de frutificação, com níveis de erros iguais a 0,20 . . . . .

10 Linhas de decisão do plano de amostragem seqüencial para frutos, com níveis de erros iguais a $0,20 \ldots \ldots \ldots \ldots \ldots$. . . . . . . . . . . . . . . 42

11 Curva característica operacional do plano de amostragem seqüencial para frutos, com níveis de erros iguais a 0,20 
12 Curva do número médio de unidades amostrais do plano de amostragem seqüencial para frutos, com níveis de erros iguais a $0,20 \ldots \ldots \ldots \ldots$. . . . . . . 45

13 Linhas de decisão do plano de amostragem seqüencial para ramos no período vegetativo, com níveis de erros iguais a $0,10 \ldots \ldots$. . . . . . . . . . . . 46

14 Curva característica operacional do plano de amostragem seqüencial para ramos no período vegetativo, com níveis de erros iguais a $0,10 \ldots \ldots$. . . . . . 48

15 Curva do número médio de unidades amostrais do plano de amostragem seqüencial para ramos no período vegetativo, com níveis de erros iguais a $0,10 \ldots \ldots$

16 Linhas de decisão do plano de amostragem seqüencial para ramos no período de frutificação, com níveis de erros iguais a $0,10 \ldots$. . . . . . . . . . . . . . 49

17 Curva característica operacional do plano de amostragem seqüencial para ramos no período de frutificação, com níveis de erros iguais a 0,10 . . . . . . . . . . . . 51

18 Curva do número médio de unidades amostrais do plano de amostragem seqüencial para ramos no período de frutificação, com níveis de erros iguais a 0,10 . . . . . 51

19 Linhas de decisão do plano de amostragem seqüencial para frutos, com níveis de

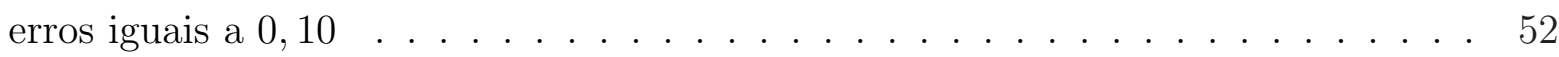

20 Curva característica operacional do plano de amostragem seqüencial para frutos, com níveis de erros iguais a $0,10 \ldots \ldots \ldots \ldots$. . . . . . . . . . 54

21 Curva do número médio de unidades amostrais do plano de amostragem seqüencial para frutos, com níveis de erros iguais a $0,10 \ldots \ldots \ldots$. . . . . . . . 54 


\section{LISTA DE TABELAS}

Página

1 Datas de amostragens e variedades de manga em cada talhão da Fazenda Bela Vista, Jaboticabal, SP, $2003 \ldots \ldots \ldots \ldots$

2 Datas de amostragens e variedades de manga em cada talhão da Fazenda Santa Maria, Jaboticabal, SP, $2003 \ldots \ldots \ldots \ldots$

3 Número de plantas amostradas em função do tamanho dos talhões . . . . . . . . . 30

4 Ajuste da distribuição binomial aos dados para a amostragem realizada na Fazenda Bela Vista, onde $\chi^{2}$ calc. méd. é o valor de $\left(\chi^{2}\right.$ calculado na comparação entre as médias observadas em cada data de amostragem com a média da distribuição binomial) e $\chi^{2}$ tabela é o valor de ( $\chi^{2}$ tabelado com nível de probabilidade igual a

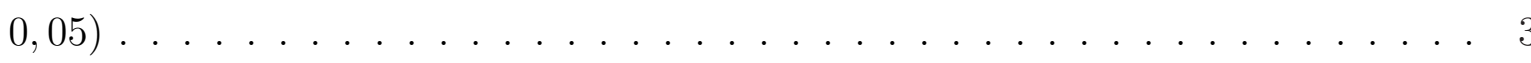

5 Ajuste da distribuição binomial aos dados para a amostragem realizada na Fazenda Santa Maria, onde $\chi^{2}$ calc. méd. é o valor de $\left(\chi^{2}\right.$ calculado na comparação entre as médias observadas em cada data de amostragem com a média da distribuição binomial) e $\chi^{2}$ tabela é o valor de $\left(\chi^{2}\right.$ tabelado com nível de probabilidade igual a

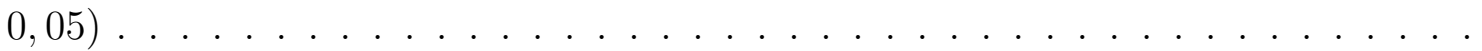

6 Plano de amostragem seqüencial para o monitoramento da cochonilha-branca em ramos no período vegetativo, com $\alpha=\beta=0,20 \ldots \ldots$. . . . . . . 37

7 Plano de amostragem seqüencial para o monitoramento da cochonilha-branca em ramos no período de frutificação, $\operatorname{com} \alpha=\beta=0,20$. P.O. (Plantas observadas), L.I. (Limite inferior), $N^{o}$ Ac. (Número acumulado de plantas infestadas) e L.S. (Limite superior) . . . . . . . . . . . . . . . . . . 40

8 Plano de amostragem seqüencial para o monitoramento da cochonilha-branca em frutos, com $\alpha=\beta=0,20 \ldots \ldots \ldots \ldots \ldots \ldots$ 
9 Plano de amostragem seqüencial para o monitoramento da cochonilha-branca em ramos no período vegetativo, $\operatorname{com} \alpha=\beta=0,10$. P.O. (Plantas observadas), L.I. (Limite inferior), $N^{o}$ Ac. (Número acumulado de plantas infestadas) e L.S. (Limite

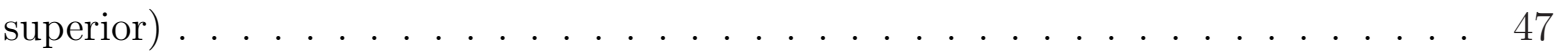

10 Plano de amostragem seqüencial para o monitoramento da cochonilha-branca em ramos no período de frutificação, com $\alpha=\beta=0,10$. P.O. (Plantas observadas), L.I. (Limite inferior), $N^{o}$ Ac. (Número acumulado de plantas infestadas) e L.S. (Limite superior $) \ldots \ldots \ldots \ldots \ldots$

11 Plano de amostragem seqüencial para o monitoramento da cochonilha-branca em frutos, com $\alpha=\beta=0,10$. P.O. (Plantas observadas), L.I. (Limite inferior), $N^{o}$ Ac. (Número acumulado de plantas infestadas) e L.S. (Limite superior) . . . . . . 53 


\title{
AMOSTRAGEM SEQÜENCIAL (PRESENÇA-AUSÊNCIA) PARA O MONITORAMENTO DA COCHONILHA-BRANCA AULACASPIS TUBERCULARIS (NEWSTEAD) (HEMIPTERA: DIASPIDIDAE) NA CULTURA DA MANGA
}

\author{
Autor: WILLIAN VICTOR SACONATO \\ Orientadora: $\operatorname{Prof}^{\underline{a}} \operatorname{Dr}^{\underline{a}}$ SÔNIA MARIA DE STEFANO PIEDADE
}

\section{RESUMO}

Foi feita uma revisão bibliográfica sobre a aplicação da amostragem seqüencial em manejos de pragas. A metodologia para o desenvolvimento de planos de amostragem seqüencial, foi baseada na teoria de Wald. Verificou-se que a distribuição binomial ajustou-se aos dados de presença-ausência da cochonilha-branca Aulacaspis tubercularis na cultura da manga, obtidos em duas propriedades localizadas na região de Jaboticabal, interior do estado de São Paulo. Seis planos de amostragem seqüencial foram elaborados para o monitoramento dessa praga, três com níveis de erros iguais a 0,20 e outros três com níveis de erros iguais a 0,10. Dentre esses, foram elaborados: dois planos para a amostragem em ramos durante o período vegetativo, dois para a amostragem em ramos durante o período de frutificação e outros dois planos para a amostragem em frutos. Nos planos de amostragem seqüencial para ramos no período vegetativo foram utilizados níveis de segurança iguais a 0,35 e níveis de 
dano econômico iguais a 0,50. Já no período de frutificação, houve um rigor maior quanto ao uso desses níveis, devido à presença de frutos. Foram utilizados níveis de segurança iguais a 0,20 para ramos e 0,05 para frutos, e níveis de dano econômico iguais a 0,30 e 0,15 para ramos e frutos, respectivamente. Foram construídas tabelas para o processo de amostragem em campo, facilitando assim, o trabalho do amostrador. Com o uso dessas tabelas o produtor poderá decidir rapidamente se há, ou não, a necessidade de se realizar o controle dessa praga. 


\title{
SEQUENTIAL SAMPLING (PRESENCE-ABSENCE) FOR MONITORING OF MANGO SCALE AULACASPIS TUBERCULARIS (NEWSTEAD) (HEMIPTERA: DIASPIDIDAE) ON MANGO CROP
}

\author{
Author: WiLLIAN ViCTOR SACONATO

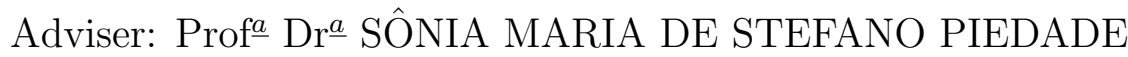

\section{SUMMARY}

A bibliographical revision on the application of the sequential sampling in management of pests was made. The methodology for the development of plans of sequential sampling, was based on the theory of Wald. It was verified that the binomial distribution adjusted to data of presence-absence of the mango scale Aulacaspis tubercularis on mango crop, gotten in two properties located at the region of Jaboticabal, interior of the state of São Paulo. Six plans of sequential sampling was elaborated for the monitoring of this pest, three with errors levels equal to 0,20 and others three with errors levels equals to 0,10. Amongst these, they was elaborated: two plans for the sampling in branches during the vegetative period, two for the sampling in branches during the period of fructification and others two plans for the sampling in fruits. In the plans of sequential sampling for branches in the vegetative period security level equal to 0,35 and economic damage level equal to 0,50 had been used. Already in longer period of fructification, had a bigger severity how much to the use of these levels, due to presence of fruits. Security levels equals to 0,20 for branches and 0,05 for fruits, 
and economic damage levels equals to 0,30 and 0,15 for branches and fruits, respectively, had been used. Tables for the sampling process in field, thus facilitating, the sampler's work was constructed. With the use of these tables the producer will be able to decide quickly has itself, or not, the necessity of if carrying through the control from this pest. 


\section{INTRODUÇÃO}

A manga (Mangifera indica), espécie originária da Ásia, atualmente é produzida em mais de 100 países, sendo a maior parte em países em desenvolvimento, como Brasil, China, Índia, México e Paquistão (Pizzol et al.) ${ }^{1}$ mencionados por Perosa \& Pierre (2002), e é hoje, uma das frutas tropicais mais consumidas no Brasil. Esses autores ainda destacam que a manga é de grande importância para a economia brasileira, pois, em 1995 ela representou $20 \%$ do volume total de frutas exportadas pelo Brasil, perdendo apenas para a laranja.

A comercialização da manga no mercado interno brasileiro centraliza-se em uma única variedade muito produtiva, a norte-americana Tommy Atkins, representando $79 \%$ da área plantada no Brasil. Essa variedade tem casca de coloração vermelha e apresenta-se pobre nos atributos de qualidade de polpa, como sabor e ausência de fibras. Merecem ainda destaque as cultivares Hadden, Keitt, Vandike e também a Palmer, que nos últimos anos vem adquirindo boa aceitação.

A região Nordeste é a principal região produtora de manga do país com $53 \%$ da produção nacional, sendo o Vale do São Francisco o eldorado brasileiro da produção e exportação. Dentre os estados podem-se destacar São Paulo (maior produtor) e Bahia.

No Estado de São Paulo, a manga está distribuída em quase todos os municípios, mas podem-se destacar as regiões de Andradina, Catanduva, General Salgado, Jaboticabal e São José do Rio Preto, que correspondem a 57\% da área total, e vem apresentando números crescentes na produção, devido à melhoria de qualidade e o aumento das exportações.

A implantação de uma estratégia coordenada de produção e distribuição ao longo do ano poderia aumentar ainda mais a participação da manga brasileira no mercado externo (Perosa \& Pierre, 2002).

Devido às condições climáticas, o cultivo da manga no estado de São Paulo

${ }^{1}$ PIZZOL, S.J.; MARTINES FILHO, J.G.; SILVA, T.H.S.; GONÇALVES, G. O mercado da manga no Brasil: aspectos gerais. Preços Agrícolas, v.12, n.142, p.34, 1998. 
está sujeito a diversos tipos de insetos/pragas e doenças, que afetam os pomares em todas as fases (vegetativa e de frutificação). No intuito de evitar isso, muitos produtores têm feito uso de controle químico de forma inadequada, podendo causar à cultura, prejuízos consideráveis. Como solução para esse tipo de problema foi desenvolvida uma técnica denominada Manejo Integrado de Pragas (MIP), que segundo Souza Filho et al. (2004) representa um avanço significativo como sistema racional de controle de pragas em frutíferas, pois tem como principal objetivo, a utilização mínima de agroquímicos, no sentido de amenizar problemas de contaminação do ambiente e, consequentemente, diminuir as taxas de resíduos no produto final, garantindo uma melhor qualidade de vida, tanto para o produtor como para o consumidor.

O Manejo Integrado de Pragas tem sido muito utilizado para o monitoramento de pragas em diversas culturas tais como o bicho-mineiro do cafeeiro (Perileucoptera coffeella) (Villacorta \& Tornero, 1982; Bearzoti \& Aquino, 1994; Vieira Neto et al., 1999), os ácaros da falsa ferrugem (Phyllocoptruta oleivora) e da leprose (Brevipalphus phoenicis) nos citros (Gravena et al., 1988), Eumargarodes laingi e Promargarodes spp. na cana-de-açúcar (Walker \& Allsopp, 1993), Tuta absoluta em tomateiro estaqueado (Gomide et al., 2001), a lagarta do milho (Spodoptera frugiperda) (Farias et al., 2001a, $2001 b$ e 2001c), e a lagarta do algodão (Alabama argillacea) (Fernandes et al., 2003).

Na cultura da manga, diversas pragas têm feito com que os produtores utilizem inadequadamente defensivos agrícolas, acarretando em um alto custo de produção. Deve-se dar um destaque especial à cochonilha-branca (Aulacaspis tubercularis) NEWSTEAD, considerada uma praga-chave dessa cultura. No MIP as pragas-chave são aquelas que de uma maneira geral estão presentes na cultura em níveis populacionais relativamente altos e provocam um tipo de injúria que pode acarretar perdas significativas, portanto, são aquelas para as quais devem-se orientar o monitoramento e o controle, pois sãos as mais importantes e que causam os maiores prejuízos e, muitas vezes, controlando-as já se controla as demais (Crocomo, 1990; Silveira Neto, 1990).

Um manejo integrado requer um conhecimento do inseto/praga, seu nível de controle e métodos de amostragem para a tomada de decisões. O monitoramento do nível populacional da praga é necessário, observando alguns índices importantes, tais como o nível de dano econômico $(N D E)$, menor densidade populacional que causa prejuízo econômico, compensando, portanto, a aplicação de medidas de controle e o nível de limiar econômico, nível populacional no qual medidas devem ser tomadas para se evitar que a população do 
inseto/praga, atinja o NDE (Silveira Neto et al., 1976).

O MIP baseia-se na aplicação de princípios ecológicos visando maior economia na exploração agrícola, ao contrário dos programas tradicionais de controle de pragas que visam apenas a economia. No que diz respeito ao Manejo Integrado de Pragas, a tomada de decisão é um aspecto chave e básico para se decidir sobre a necessidade, ou não, de alguma ação de controle com base nas informações obtidas. Pode-se ainda, segundo Binns \& Nyrop ${ }^{2}$ mencionados por Scorza Junior et al. (1996), destacar dois pontos de vital importância para a tomada de decisão da realização, ou não, do controle: a amostragem e o conhecimento do nível de dano econômico.

Desenvolvida por Wald em 1943, durante a II Guerra Mundial, a amostragem seqüencial passou a ter uso extensivo em estudos entomológicos a partir da década de 50, intensificando-se a partir dos anos 70, quando ganhou impulso no campo prático com trabalhos de Ingram \& Green ${ }^{3}$ mencionados por Farias et al. (2001b), Pieters \& Sterling (1975), Sterling (1975), Shepard \& Carner (1976) e Estefanel (1977).

Essa forma de amostragem está sendo cada vez mais usada em entomologia, visando minimizar os seus custos, sem que que haja uma redução na precisão dos resultados obtidos (Taylor, 1984).

O objetivo desse trabalho consistiu em elaborar planos de amostragem seqüencial, baseados no Teste Seqüencial da Razão de Probabilidades, com níveis de erros diferentes para decidir sobre a necessidade, ou não, do controle da cochonilha-branca, sendo as unidades amostrais, classificadas em mangueiras com a presença ou ausência da cochonilha, fazendo com que o produtor obtenha um resultado confiável com mais rapidez e com o menor número de observações possível.

${ }^{2}$ BINNS, M. R.; NYROP, J. F. Sampling insect populations for the purpose of IPM decision making. Annual Review of Entomology, v.37, p.427-453, 1992.

${ }^{3}$ INGRAM, W. R.; GREEN, S. M. Sequential sampling bollworms on raingrow cotton in Botswana. Cotton Grower Review, v.49, p.265-275, 1972. 


\section{REVISÃO DE LITERATURA}

A praticidade de um programa de amostragem está baseada no equilíbrio entre a confiança estatística e os níveis de precisão e nas restrições práticas de sua aplicação, particularmente o tempo (Régnière et al., 1988).

Pode-se dizer que existem basicamente dois tipos de amostragem: a amostragem convencional e a amostragem seqüencial. A amostragem convencional caracteriza-se, principalmente, por apresentar um número fixo de unidades amostrais, determinado antes de se iniciar o procedimento de amostragem, e os resultados são avaliados após o seu término. Já a amostragem seqüencial é um método estatístico caracterizado por utilizar um número variável de observações.

A amostragem seqüencial é um procedimento no qual observações são tiradas em seqüência e as decisões são feitas após a leitura de cada unidade amostral. Nesse tipo de amostragem, a avaliação pode classificar rapidamente a população do organismo tratado em grandes categorias como baixa, média ou alta, ou ainda indicar se é necessário adotar alguma medida de controle, ou não, (Lynch et al., 1990). Assim, de acordo com Wald (1945), o momento de terminar a amostragem e tomar uma decisão depende, em cada etapa, dos resultados obtidos a cada momento.

O crescente interesse pela utilização da amostragem seqüencial está relacionado com o desenvolvimento de programas de Manejo Integrado de Pragas, e assim tem sido muito aplicada para avaliar a infestação de insetos nas culturas e decidir sobre o uso, ou não, de inseticidas. Essa forma de amostragem é apropriada para aplicação em MIP, pois o objetivo está essencialmente em propor a classificação de populações, a estimar parâmetros da população. Segundo Gravena (1984), o MIP tem como estrutura fundamental o uso seletivo de defensivos aos inimigos naturais das pragas combinado a um programa prático de monitoramento, com o objetivo de reduzir os custos de tratamento contra pragas, sem afetar o vigor da planta e a qualidade do fruto. 
De acordo com Brader ${ }^{4}$ mencionado por Gravena (1984) o controle de pragas e doenças se assenta nos requisitos econômicos, ecológicos e toxicológicos reservando prioridade aos fatores naturais limitantes e aos níveis limiares de dano e segundo Bosch et al.(1985) visa, também, a uma eficiência nos resultados.

A finalidade da amostragem em programas de manejo de pragas consiste em determinar se os níveis populacionais das pragas estão acima ou abaixo dos níveis de ação especificados (Luna et al., 1983).

A amostragem seqüencial é viável quando:

i. a população está reunida na área delimitada pela cultura,

ii. a avaliação das unidades amostrais, ou seja, a contagem do número de insetos ou a verificação de existência, ou não, de danos, é feita imediatamente, sem necessitar de análises de laboratório ou outro processo moroso, e

iii. quando não se está interessado em conhecer o parâmetro da infestação, mas somente se a infestação atinge determinado nível a partir do qual a aplicação de inseticida deve ser executada.

Devido à utilização de métodos de amostragem convencionais, para os quais o número ou tamanho da amostra é fixo, é necessário muito tempo para a tomada de decisão. Por isso, às vezes, a tomada de decisão de controlar, ou não, a praga é feita de forma empírica (Bianco, 1995).

A conseqüencia dessa atitude resulta no uso indevido de produtos químicos, por decisões precipitadas, que oneram o custo da produção, promovendo o desequilíbrio do agroecossistema e outros efeitos colaterais (Farias et al., 2001b).

Com o uso da amostragem seqüencial ocorre uma diminuição do tamanho da amostra implicando em economia. Além da obtenção do resultado mais rapidamente, este método de amostragem oferece ao agricultor uma alta confiabilidade estatística, e segundo Sterling (1975), resulta em uma redução no tempo e no custo da aplicação do plano em relação à amostragem convencional.

Para elaborar um plano de amostragem seqüencial duas metodologias têm sido

\footnotetext{
${ }^{4}$ BRADER, L. Integrated control, a new approach in crop protection. In: C.R. 5e Symp. Lutte integrée en vergers. OILB SROP, 1975. p.9-16.
} 
muito utilizadas: o Intervalo de Confiança de Iwao ${ }^{5}$ mencionado por Nyrop \& Simmons (1984), Régnière et al. (1988), Wright et al. (1990) e Vieira Neto et al. (1999) e o Teste Seqüencial da Razão de Probabilidades de Wald (1945 e 1947).

Esse intervalo de confiança está relacionado com o uso do Teorema do Limite Central. Trata-se de um intervalo de confiança em torno da média (nível de controle), o qual gera duas curvas divergentes chamadas linhas de decisão do plano, e independe do modelo probabilístico. Faz uso da Lei da Potência de Taylor ${ }^{6}$ mencionado por Maiteki \& Lamb (1987), Shelton et al. (1987), Wright et al. (1990), Peng \& Brewer (1995) e Farias et al. (2001a). A grande desvantagem desta metodologia é a falta de conhecimento dos erros que podem ser cometidos através de sua aplicação (Vieira Neto et al., 1999).

O Teste Seqüencial da Razão de Probabilidades (TSRP) de Wald (1945 e 1947) baseia-se na Curva Característica Operacional, que fornece a proteção que o plano oferece contra decisões erradas e está estreitamente relacionada com o poder do teste; e na Curva do Número Médio de Unidades Amostrais a qual apresenta o tamanho médio necessário de amostras para tomar uma decisão.

De acordo com Barbosa (1985), três requisitos básicos são necessários para elaborar um plano de amostragem seqüencial:

1. O tipo de função matemática que melhor descreva a distribuição das contagens de insetos ou lesões por eles causadas ou qualquer outra variável relacionada;

2. Nível de limiar econômico ou nível de dano na forma de duas densidades populacionais críticas, e

3. Seleção de níveis máximos de probabilidade de cometer erros na tomada de decisão sobre densidades populacionais, isto é, probabilidades $\alpha$ e $\beta$ de predizer uma densidade populacional não prejudicial como sendo prejudicial (erro tipo $I$ ), e a de predizer uma densidade populacional prejudicial como sendo não prejudicial (erro tipo $I I$ ), respectivamente.

Segundo Wald (1945), para desenvolver um plano de amostragem seqüencial, inicialmente é necessário conhecer a distribuição espacial dos insetos/pragas que, segundo

\footnotetext{
${ }^{5}$ IWAO, S. A new method of sequential sampling to classify populations relative to a critical density.
}

Research on Population Ecology, v.16, p.281-288, 1975.

${ }^{6}$ TAYLOR, L. R. Aggregation, variance and the mean. Nature, v.189, p.732-735, 1961. 
Estefanel (1977), depende da unidade amostral, do comportamento da espécie e do tipo de avaliação que é feita, sendo essa, preferencialmente, indexada por um único parâmetro (Nyrop \& Wright, 1985; Hollingsworth \& Gatsonis, 1990).

De acordo com Taylor (1984), a distribuição dos organismos no espaço pode ser classificada em três disposições básicas que descrevem os arranjos espaciais ocupados pelos insetos: disposição aleatória, disposição uniforme e disposição agregada. Tais distribuições são denominadas Poisson, binomial e binomial negativa, respectivamente.

Segundo Perecin \& Barbosa (1992), dentre todas as distribuições de probabilidade, as mais utilizadas para estudar a disposição espacial dos insetos são: Poisson, binomial e binomial negativa. A distribuição normal também já foi utilizada para a elaboração de planos de amostragem seqüencial para controle de insetos, mas dentre as citadas, esta foi a menos utilizada.

Estefanel (1977) verificou que a distribuição binomial negativa se ajustou a dados de contagens de lagartas da soja, concordando com Evans (1953), que obteve a mesma conclusão quanto às contagens de insetos existentes numa determinada parcela. Essa verificação discordou dos resultados a que chegaram Shepard \& Carner (1976) nos Estados Unidos, os quais concluíram que a distribuição de Poisson obteve melhor ajuste. Mesmo chegando a essa conclusão quanto às contagens de lagartas da soja, Shepard \& Carner (1976) afirmaram que a distribuição binomial negativa ajusta-se à maioria dos dados de contagens de insetos.

Segundo um estudo realizado por Dantas (2002) sobre a distribuição espacial da lagarta do minador-dos-citros em pomares de laranjeira 'Pera-rio', dentre as distribuições estudadas: Poisson, binomial e binomial negativa, esta última foi o modelo que obteve melhor ajuste, concordando com Fernandes (2002), que obteve a mesma conclusão quanto às lagartas na cultura do algodoeiro.

Nos casos em que as populações dos insetos são difíceis de serem quantificadas, o método recomendado é a amostragem seqüencial (ou amostragem binomial) presença-ausência (Wilson \& Room, 1983). Segundo Ingram \& Green ${ }^{7}$ mencionados por Farias et al. (2001b), este método leva em conta apenas se o indivíduo está, ou não, presente na planta, independente da quantidade.

Para elaborar um plano seqüencial binomial é suficiente verificar a presença ou a ausência do inseto nas unidades amostrais, sem a necessidade de contar o número de

${ }^{7}$ INGRAM, W. R.; GREEN, S. M. Sequential sampling bollworms on raingrow cotton in Botswana. Cotton Grower Review, v.49, p.265-275, 1972. 
indivíduos por planta para considerá-la infestada (Sterling ${ }^{8}$; Sterling et al. ${ }^{9}$ ) mencionados por Gravena et al. (1988).

Barbosa (1982) ${ }^{10}$ mencionado por Farias et al. (2001b), cita a distribuição binomial como sendo a mais indicada para descrever a proporção de plantas atacadas pela lagarta Spodoptera frugiperda na cultura do milho.

De acordo com Bechinski \& Stoltz (1985), as principais vantagens da amostragem presença-ausência são: facilidade e, principalmente, rapidez na amostragem.

\subsection{Nível de Limiar Econômico}

Em um programa de manejo integrado de pragas é de fundamental importância a existência de um sistema efetivo de amostragem para determinar a intensidade de ataque da praga à cultura. É necessário distinguir os diferentes níveis de infestação e o grau em que esses níveis influenciam na cultura, a fim de obter um determinado nível de dano.

Segundo Hillhouse \& Pitre $^{11}$ mencionados por Gomide et al. (2001), para obter tais informações, é necessário estabelecer, antecipadamente, a precisão e a eficiência das técnicas de amostragem.

A escolha da técnica de amostragem é baseada no objetivo de determinar, com certa precisão, a necessidade, ou não, de controle da praga, levando em conta o aspecto econômico (Villacorta \& Tornero, 1982).

No Brasil, o nível de limiar econômico tem sido um dos entraves para o desenvolvimento de planos de amostragem seqüencial, pois requer estudos de longa duração, envolvendo prejuízos da praga, custos de controle e valor da produção. Barbosa (1992) sugere que, em vez de esperar até que sejam coletados todos os dados necessários para se iniciar um programa de manejo de pragas, empregue-se a melhor informação existente e os conhecimentos

\footnotetext{
${ }^{8}$ STERLING, W. L. Sequential decision plans for the management of cotton arthropods in south-east Queensland. Australian Journal Economic, v.1, p.265-274, 1976.

${ }^{9}$ STERLING, W. L.; BLEICHER, E.; JESUS, F. M. M. Um programa de manejo integrado para insetos do algodoeiro no Nordeste do Brasil usando amostragem seqüencial. Anais da Sociedade Entomológica do Brasil, v.12, n.1, p.85-98, 1983.

${ }^{10}$ BARBOSA, J. C.; PERECIN, D. Modelos probabilísticos para a distribuição de lagartas de Spodoptera frugiperda (J. E. Smith, 1797) na cultura do milho. Científica, v.10, p.181-191, 1982.

${ }^{11}$ HILlHOUSE, T. L.; PITRE, H. N. Comparison of sampling techniques to obtain measurements of insect populations on soybeans. Journal of Economic Entomology, v.67, p.411-414, 1974.
} 
de nível prático adquiridos para se elaborar um manejo experimental de pragas.

\subsection{Seleção de Níveis Máximos de Probabilidade}

Com base no erro tipo I recomenda-se realizar o controle quando não há necessidade o que pode acarretar em prejuízo ao produtor e ao meio ambiente, e com base no erro tipo II recomenda-se não realizar o controle quando este é necessário levando a perdas na produção (Vieira Neto et al., 1999). Para evitar isso, é de fundamental importância obter uma probabilidade aceitável de erro na tomada de decisão.

Desde que os valores desses erros sejam especificados com antecedência, é possível pré-determinar a precisão do plano. Além disso, de acordo com Vieira Neto et al.(1999), no dimensionamento da amostra, geralmente leva-se em conta apenas o erro tipo $I$.

\subsection{Erro de amostragem}

De acordo com Luna et al. (1983), a amostragem seqüencial é um método de alocação ótima em trabalhos de manejos, alcançando decisões baseadas em um nível de erro especificado.

Com o aumento do tamanho da amostra, apenas o erro de amostragem diminui, mas isso acontece até certo ponto a partir do qual unidades amostrais adicionais praticamente não o afetam. Erros de outra natureza têm efeitos iguais, seja a amostra pequena ou grande (Da Silva et al., 1998).

\subsection{Tamanho da amostra e tempo de amostragem}

A determinação do tamanho ou número de unidades amostrais, é necessária quando se elabora um plano de amostragem, pois está diretamente ligada à redução no custo e no tempo de realização do plano.

Planos de amostragem seqüencial podem reduzir, significativamente, o tempo e o esforço necessários para caracterizar populações, e de acordo com Shields \& Specker (1989), resultam em uma economia de $67,7 \%$ no número médio de locais visitados para a amostragem 
de batatas.

Amostras muito grandes tendem a aumentar o tempo e os custos da amostragem e amostras muito pequenas podem resultar em menor precisão, o que também é indesejável. O pesquisador está interessado em tamanhos amostrais que permitam boa precisão e economia de tempo e recursos (Da Silva et al., 1998).

Segundo Wald (1945), com o uso da amostragem seqüencial, há uma redução de 50\% no tempo de amostragem, quando comparado com o método convencional. Wald (1947) demonstrou que em certos casos, a amostragem seqüencial requer, em média, amostras de um terço $(1 / 3)$ do tamanho que seria utilizado com a amostragem de tamanho fixo.

Anscombe (1949) sugeriu que para verificar o ajustamento à distribuição binomial negativa deve-se ter pelo menos 10 unidades amostrais, mas no mesmo artigo, este autor chegou à conclusão que este tamanho era insuficiente.

Quando a densidade da praga está muito abaixo ou muito acima do nível de controle, a decisão de controlar, ou não, é tomada com menor número de observações (Hollingsworth \& Gatsonis, 1990). Segundo Pieters \& Sterling (1975), isso permite uma redução no tempo de amostragem em torno de 50\%, diminuindo o seu custo. Mesmo que níveis de densidade populacional intermediários exijam grandes amostras, uma economia substancial no tempo de amostragem pode ser obtida através do uso da amostragem seqüencial (Lynch et al., 1990).

Pelos trabalhos de Sterling (1975), com o uso da tabela de amostragem seqüencial ocorre uma redução de $86 \%$ no tamanho da amostra na coleta de artrópodos em algodoeiro, concordando com Rothrock \& Sterling ${ }^{12}$ mencionados por Gravena et al. (1988), os quais também demonstraram que ocorre uma redução de $76 \%$ no tempo de amostragem. E ainda, de acordo com estudos de Estefanel (1977) sobre o uso da amostragem seqüencial no controle das lagartas da soja no Estado do Rio Grande do Sul, utilizando amostras de 50 unidades amostrais, este pode concluir que 50 unidades foi um número muito pequeno, e que em próximos levantamentos talvez fosse mais viável utilizar um número de unidades amostrais igual ou maior do que 100.

Luna et al. (1983) chegaram à conclusão de que há uma redução significativa no número de unidades amostrais requeridas no manejo de pragas em batata.

Em uma comparação feita entre as amostragens convencional e seqüencial em

\footnotetext{
${ }^{12}$ ROTHROCK, M. A.; STERLING, W. L. Sequential sampling for arthropods of cotton its advantages over point sampling. The Southwestern Entomologist, v.7, n.2, p.70-81, 1982.
} 
pomares cítricos, foi constatada por Gravena et al. (1988) uma redução do número de frutos observados para a tomada de decisão de aproximadamente $60 \%$ a 94\%. E observou-se, também, uma acentuada redução no tempo necessário para a amostragem, que variou de aproximadamente $48 \%$ a $84 \%$.

Wright et al. (1990) afirmaram que uma das vantagens do método de amostragem seqüencial é a necessidade de um menor número de observações em relação ao método de contagem, concordando com Hollingsworth \& Gatsonis (1990), os quais recomendam um mínimo de 10 e um máximo de 50 unidades amostrais para o plano de amostragem feito para afídios em batata. Mas de acordo com Vieira Neto et al. (1999), um maior número de observações pode ser exigido quando a tomada de decisão é considerada complicada, enquanto que, nos planos convencionais o número de observações é sempre fixo, para qualquer grau de infestação

Segundo Bianco (1995) quando a disposição espacial dos insetos/pragas é do tipo ao acaso ou uniforme, o número de unidades amostrais é menor, quando comparado com a disposição agregada. O número de unidades amostrais deve ser tal que possibilite uma amostragem precisa, rápida e de baixo custo (Bliss \& Owens ${ }^{13}$; Pedigo et al. ${ }^{14}$; Pedigo ${ }^{15}$; Gusmão $^{16}$

\subsection{Teste seqüencial da razão de probabilidades}

Considere $X$ uma variável aleatória e $f(x, \theta)$ a distribuição dessa variável. Se $X$ for uma variável aleatória discreta $f(x, \theta)$ indicará a probabilidade dessa variável tomar o valor $\theta$. Se $X$ for uma variável aleatória contínua então $f(x, \theta)$ indicará uma função densidade de probabilidade.

Suponha que as hipóteses a serem testadas sejam $H_{0}: \theta=\theta_{0} \quad$ e $\quad H_{1}: \theta=\theta_{1}$.

\footnotetext{
${ }^{13}$ BLISS, C. I.; OWENS, A. R. G. Negative binomial distributions with a common $k$. Biometrika, v.45, n.1/2, p.37-58, 1958 .

${ }^{14}$ PEDIGO, L. P.; BUTIN, G. D.; BECHINSKI, E. J. Flushing technique and sequential-count plan for green cloverworm (Lepidoptera: Noctuidae) moths in soybeans. Environmental Entomology, v.11, n.6, p.1223-1228, 1982.

${ }^{15}$ PEDIGO, L. P. Entomology and pest management. New York: Macmillan, 1988. 646p.

${ }^{16}$ GUSMÃO, M. R. Avaliação de vetores de viroses, predadores e parasitóides e plano de amostragem para mosca-branca do tomateiro. Viçosa, 2000. 42f. Dissertação (Mestrado), Centro de Ciências Agrárias, Universidade Federal de Viçosa.
} 
Então a distribuição de $\mathrm{X}$ será dada por $f\left(x, \theta_{0}\right)$ quando $H_{0}$ for verdadeira e por $f\left(x, \theta_{1}\right)$ quando $H_{1}$ for verdadeira (Estefanel, 1977).

A probabilidade de obter uma amostra $x_{1}, x_{2}, \ldots, x_{m}$, sendo $m$ um valor inteiro positivo é dada por

$$
p_{1 m}=f\left(x_{1}, \theta_{1}\right) \cdot f\left(x_{2}, \theta_{1}\right) \ldots f\left(x_{m}, \theta_{1}\right)
$$

quando $H_{1}$ for verdadeira, ou por

$$
p_{0 m}=f\left(x_{1}, \theta_{0}\right) \cdot f\left(x_{2}, \theta_{0}\right) \ldots f\left(x_{m}, \theta_{0}\right)
$$

quando $H_{0}$ for verdadeira.

A Razão de Probabilidades ou Razão de Verossimilhanças é definida como a seguir

$$
\frac{p_{1 m}}{p_{0 m}}=\frac{f\left(x_{1}, \theta_{1}\right)}{f\left(x_{1}, \theta_{0}\right)} \cdot \frac{f\left(x_{2}, \theta_{1}\right)}{f\left(x_{2}, \theta_{0}\right)} \cdots \frac{f\left(x_{m}, \theta_{1}\right)}{f\left(x_{m}, \theta_{0}\right)}
$$

a qual pode ser abreviada da seguinte forma

$$
\frac{p_{1 m}}{p_{0 m}}=\prod_{i=1}^{m} \frac{f\left(x_{i}, \theta_{1}\right)}{f\left(x_{i}, \theta_{0}\right)}
$$

Wald (1945) destaca que se $p_{1 m}=p_{0 m}=0$, o valor da razão $p_{1 m} / p_{0 m}$ é definido igual a 1.

Aceita-se $H_{1}$ se

$$
\frac{p_{1 m}}{p_{0 m}} \geq A
$$

Aceita-se $H_{0}$ se

$$
\frac{p_{1 m}}{p_{0 m}} \leq B
$$

Deve-se tomar uma observação adicional se

$$
B<\frac{p_{1 m}}{p_{0 m}}<A
$$

Dessa forma, o número $n$ de observações necessárias para o teste seqüencial é o menor inteiro positivo de $m$ para o qual considerar-se-á a equação (2) ou a equação (3). As constantes $A$ e $B$ são escolhidas tais que $0<B<A$ e o teste seqüencial tem o valor desejado $\alpha$ da probabilidade de ocorrer um erro tipo $I$ e o valor desejado $\beta$ da probabilidade de ocorrer um erro tipo II. Wald (1945) chama o teste definido por (2), (3) e (4), como Teste Seqüencial da Razão de Probabilidades. 


\subsection{Determinação dos valores $A$ e $B$}

Os valores de $A$ e $B$ dependem das probabilidades $\alpha$ e $\beta$, sendo que $\alpha$ representa a probabilidade de rejeitar a hipótese $H_{0}$ quando esta for verdadeira (erro tipo $I$ ) e $\beta$ representa a probabilidade de aceitar $H_{0}$ quando esta for falsa (erro tipo $I I$ ).

Considere uma amostra $x_{1}, x_{2}, \ldots, x_{n}$. Esta amostra será do tipo 0 se

$$
B<\frac{p_{1 m}}{p_{0 m}}<A
$$

em que $m=1,2, \ldots, n-1 \mathrm{e}$

$$
B \geq \frac{p_{1 n}}{p_{0 n}}
$$

e será do tipo 1 se

$$
B<\frac{p_{1 m}}{p_{0 m}}<A
$$

em que $m=1,2, \ldots, n-1 \mathrm{e}$

$$
\frac{p_{1 n}}{p_{0 n}} \geq A
$$

Uma amostra que safisfaça as inequações (5) e (6) (amostra tipo 0) leva a aceitar a hipótese $H_{0}$ e uma amostra que satisfaça (7) e (8) (amostra tipo 1) leva a aceitar $H_{1}$.

Da inequação (8) tem-se que a probabilidade de obter uma amostra do tipo 1 é pelo menos $A$ vezes maior sob a hipótese $H_{1}$ do que sob a hipótese $H_{0}$. A partir daí, podese concluir que a medida da probabilidade de todas as amostras do tipo 1 (probabilidade da amostragem seqüencial terminar com aceitação de $H_{1}$ ) é também $A$ vezes maior sob a hipótese $H_{1}$ do que sob a hipótese $H_{0}$. Essa probabilidade é igual a $\alpha$ quando $H_{0}$ for verdadeira e igual a $(1-\beta)$ quando $H_{1}$ for verdadeira.

Obtém-se então

$$
1-\beta \geq A \cdot \alpha
$$

que pode ser escrita como

$$
A \leq \frac{1-\beta}{\alpha}
$$


Pode-se dizer que $\frac{1-\beta}{\alpha}$ é um limite superior para $A$.

Analogamente pode-se deduzir um limite inferior para $B$. A partir de (6) podese dizer que a probabilidade de obter uma amostra do tipo 0 é no máximo $B$ vezes maior sob a hipótese $H_{1}$ do que sob a hipótese $H_{0}$. Conclui-se, também, que a probabilidade de encerrar a amostragem seqüencial com aceitação de $H_{0}$ é no máximo $B$ vezes maior quando $H_{1}$ for verdadeira do que quando $H_{0}$ for verdadeira. Essa probabilidade é igual a $(1-\alpha)$ quando $H_{0}$ for verdadeira e igual a $\beta$ quando $H_{1}$ for verdadeira.

Obtém-se daí

$$
\beta \leq(1-\alpha) \cdot B
$$

a qual pode ser escrita como

$$
B \geq \frac{\beta}{1-\alpha}
$$

Logo $\frac{\beta}{1-\alpha}$ é um limite inferior para $B$.

Wald (1945) afirma que os seguintes valores de $A$ e $B$ podem ser tomados

$$
A=\frac{1-\beta}{\alpha}
$$

e

$$
B=\frac{\beta}{1-\alpha}
$$

Segundo Wald (1945), com o uso dessas equações, o erro tipo $I$, ou seja, a probabilidade de rejeitar $H_{0}$ quando esta for verdadeira, não será superior a $\alpha$ e o erro tipo $I I$, ou seja, a probabilidade de aceitar $H_{0}$ quando esta for falsa, não será superior a $\beta$.

Wald (1945) propõe que os valores de $A$ e $B$ possam ser obtidos pelas equações (13) e (14). A única desvantagem possível em se usarem essas equações no lugar das inequações (9) e (11) é que o número esperado de observações necessárias para se tomar uma decisão pode se tornar maior. Mas esse procedimento pode ser levado em consideração já que em pesquisas agrícolas a amostragem não é excessivamente dispendiosa (Estefanel, 1977). 


\subsection{Probabilidade de aceitar $H_{0}$ ou $H_{1}$ quando alguma terceira hipótese $H$ é verdadeira}

Seja $H$ a hipótese de que a distribuição de $X$ é dada por $f(x)$. De acordo com Wald (1945) pode-se assumir que essa hipótese seja verdadeira.

Seja $\gamma$ a probabilidade de que o teste seqüencial da razão de probabilidades levará à aceitação de $H_{1}$. Neste caso, se $H=H_{0}$, então $\gamma=\alpha$ e se $H=H_{1}$, então $\gamma=1-\beta$.

Denota-se $\ln \frac{f_{1}\left(x_{i}\right)}{f_{0}\left(x_{i}\right)}$ por $Z_{i}(i=2, \ldots, \infty)$ que é uma seqüencia de variáveis aleatórias independentes cada uma tomando alguma distribuição. Seja $Z_{(j)}$ a soma dos primeiros $j$ elementos da seqüencia $Z_{i}$, ou seja,

$$
Z_{(j)}=Z_{1}+\ldots+Z_{j} \quad \text { para }(j=1,2, \ldots, \infty) .
$$

Seja $n$ o menor inteiro positivo para o qual seja considerado ou $\quad Z_{(n)} \geq \ln A$ ou $Z_{(n)} \leq \ln B$. Se $\ln B<Z_{m}<\ln A$ para $(m=1,2, \ldots, \infty)$ pode-se dizer que $n \rightarrow \infty$, sendo $n$ o número de observações necessárias para o teste seqüencial da razão de probabilidades. De acordo com as equações (13) e (14), pode-se tomar $A=\frac{1-\beta}{\alpha}$ e $B=\frac{\beta}{1-\alpha}$. Desde que $B$ seja menor do que $A$, considerar-se-ão somente os valores de $\alpha$ e $\beta$ para o qual $\frac{1-\beta}{\alpha}>\frac{\beta}{1-\alpha}$. Esta inequação é equivalente a dizer que $\alpha+\beta<1$, a qual torna implícito que $B<1$ e $A>1$. Então assumir-se-á que $B<1, A>1$ e também que a variância de $Z_{i}$ é diferente de zero.

De acordo com Wald (1945) pode-se considerar $P(n \rightarrow \infty)=0$. Portanto, a probabilidade é igual a um de que a amostragem seqüencial seja eventualmente terminada. Neste caso, está implícito que a probabilidade de aceitação de $H_{0}$ é igual a $(1-\gamma)$.

Toma-se $Z$ como uma variável aleatória cuja distribuição é igual às distribuições gerais das variáveis $Z_{i} \quad(i=1,2, \ldots, \infty)$. Seja $\varphi(t)$ a função geradora de momentos de $Z$, ou seja,

$$
\varphi(t)=E\left(e^{Z t}\right)
$$

Considere um valor $h$ tal que $h \neq 0 \quad$ e $\quad \varphi(h)=1$. A partir daí obtém-se

$$
E\left(e^{Z_{(n)} h}\right)=1
$$

Seja $E^{\prime}$ o valor esperado condicional de $e^{Z_{(n)} h}$ sob a restrição de que $H_{0}$ é aceito, 
isto é, de que $Z_{(n)} \leq \ln B$, e seja $E^{\prime \prime}$ o valor esperado condicional de $e^{Z_{(n)} h}$ sob a restrição de que $H_{1}$ é aceito, isto é, de que $Z_{(n)} \geq \ln A$. Então, da equação (15), tem-se que

$$
(1-\gamma) E^{\prime}+\gamma E^{\prime \prime}=1
$$

ou ainda

$$
\gamma=\frac{1-E^{\prime}}{E^{\prime \prime}-E^{\prime}}
$$

Wald (1945) ainda afirma que, se ambos os valores de $E_{Z}$ e a variância de $Z$ são pequenas, então quando $f_{1}(x)$ (função de distribuição de $X$ sob a hipótese $H_{1}$ ) for próximo de $f_{0}(x)$ (função de distribuição de $X$ sob a hipótese $H_{0}$ ), $E^{\prime}$ e $E^{\prime \prime}$ estarão próximos de $B^{h}$ e $A^{h}$, respectivamente. Portanto, uma boa aproximação para $\gamma$ é dada por

$$
\bar{\gamma} \sim \frac{1-B^{h}}{A^{h}-B^{h}}
$$

Verifica-se ainda que $h=1$ se $H=H_{0}$, e que $h=-1$ se $H=H_{1}$. A diferença $\bar{\gamma}-\gamma$ aproxima-se de zero se ambas a média e a variância de $Z$ converge para zero (Wald, 1945).

\subsection{Número esperado de observações necessárias para se tomar uma decisão}

Wald (1945) considera

$$
Z=\ln \frac{f_{1}(x)}{f_{0}(x)}, \quad Z_{i}=\ln \frac{f_{1}\left(x_{i}\right)}{f_{0}\left(x_{i}\right)} \quad(i=1,2, \ldots, \infty)
$$

e $n$ o número de unidades amostrais necessárias para o teste seqüencial, ou seja, $n$ é o menor inteiro positivo para o qual $Z_{(n)}=Z_{1}+Z_{2}+\ldots+Z_{n}$ é $\geq \ln A$ ou $\leq \ln B$. Para determinar o valor esperado de $n, E(n)$, sob alguma hipótese $H$, toma-se um inteiro positivo fixo $N$. A soma $Z_{(N)}=Z_{1}+Z_{2}+\ldots+Z_{N}$ pode ser dividida em duas partes como a seguir

$$
Z_{(N)}=Z_{(n)}+Z_{(n)}^{\prime}
$$

em que $Z_{(n)}^{\prime}=Z_{n+1}+Z_{n+2}+\ldots+Z_{N} \quad$ se $\quad n \leq N \quad$ e $\quad Z_{(n)}^{\prime}=Z_{(N)}-Z_{(n)} \quad$ se $n>N$. Tomando o valor esperado de ambos os lados de (17) obtém-se 


$$
N E_{Z}=E\left(Z_{(n)}\right)+E\left(Z_{(n)}^{\prime}\right)
$$

Desde que a probabilidade de $n>N$ converge para zero quando $\quad N \rightarrow \infty$, e que $\left|Z_{(n)}^{\prime}\right|<2(\ln A+|\ln B|)$ se $n>N$, obtém-se

$$
\lim _{N \rightarrow \infty}\left[E\left(Z_{(n)}^{\prime}\right)-E(N-n) E_{Z}\right]=0
$$

Das equações (18) e (19) segue que

$$
E\left(Z_{(n)}\right)=E_{n} E_{Z}
$$

Portanto,

$$
E_{n}=\frac{E\left(Z_{(n)}\right)}{E_{Z}} .
$$

Seja $E^{\prime}\left(Z_{(n)}\right)$ o valor esperado condicional de $Z_{(n)}$ sob a restrição de que a análise seqüencial leva à aceitação de $H_{0}$, isto é, que $Z_{(n)} \leq \ln B$. Da mesma forma, considerase $E^{\prime \prime}\left(Z_{(n)}\right)$ o valor esperado condicional de $Z_{(n)}$ sob a restrição de que $H_{1}$ é aceito, isto é, que $Z_{(n)} \geq \ln A$. Tomando $\bar{\gamma}$ a probabilidade de que $Z_{(n)} \geq \ln A$, pode-se escrever

$$
E\left(Z_{(n)}\right)=(1-\bar{\gamma}) E^{\prime}\left(Z_{(n)}\right)+\bar{\gamma} E^{\prime \prime}\left(Z_{(n)}\right)
$$

Substituindo (20) em (21), chega-se a

$$
E_{n}=\frac{(1-\bar{\gamma}) E^{\prime}\left(Z_{(n)}\right)+\bar{\gamma} E^{\prime \prime}\left(Z_{(n)}\right)}{E_{Z}} .
$$

Segundo Wald (1945) os valores exatos de $E\left(Z_{(n)}\right)$ e $E_{n}$, podem ser obtidos se $Z$ tomar somente um inteiro múltiplo de uma constante $k$, desde que a distribuição de probabilidade de $Z_{(n)}$ tenha sido obtida. Se $Z$ não satisfaz as restrições mencionadas anteriormente, ainda assim pode-se obter uma aproximação para o valor de $E\left(Z_{(n)}\right)$, desde que a distribuição de $Z$ possa ser aproximada para algum grau desejado por uma distribuição discreta e se a constante $k$ escolhida for suficientemente pequena.

Se $\left|E_{Z}\right|$ e o desvio-padrão de $Z$ são pequenos, então $E^{\prime}\left(Z_{(n)}\right)$ é muito próximo ou igual a $\ln B$ e $E^{\prime \prime}\left(Z_{(n)}\right)$ é muito próximo ou igual a $\ln A$. Portanto escreve-se (22) da seguinte forma 


$$
E_{n} \sim \frac{(1-\bar{\gamma}) \ln B+\bar{\gamma} \ln A}{E_{Z}}
$$

\subsection{Testando uma determinada hipótese $H_{0}$ contra uma hipótese alternativa $H_{1}$}

Seja $f(x, \theta)$ a função densidade de probabilidade de uma variável aleatória $X$, sendo $\theta$ um parâmetro desconhecido. Suponha-se que seja necessário testar uma hipótese $H_{0}: \theta=\theta_{0}$ e que os valores alternativos de $\theta$ sejam restritos a $\theta>\theta_{0}$. Assume-se que um teste seqüencial seja necessário tal que a probabilidade de um erro do tipo $I$ seja igual a $\alpha$.

Segundo Wald (1945), a probabilidade de ocorrer um erro tipo II não é mais somente um valor, mas uma função do verdadeiro valor de $\theta$. Se $f(x, \theta)$ é uma função contínua de $x$ e $\theta$, então a probabilidade de um erro tipo $I I$ será próximo de $(1-\alpha)$ se o verdadeiro valor de $\theta$ for próximo de $\theta_{0}$. Portanto, se $\alpha$ é pequeno, a probabilidade de um erro tipo $I I$ é necessariamente maior quando o verdadeiro valor de $\theta$ for próximo de $\theta_{0}$. Na prática, não se atenta para o caso em que a probabilidade de um erro tipo II seja alta quando o verdadeiro valor de $\theta$ for próximo de $\theta_{0}$, pois o erro cometido pela aceitação de $\theta_{0}$ é considerado de pequena importância (Wald, 1945). No entanto, haverá um valor $\theta_{1}>\theta_{0}$ tal que desejarse-á que a probabilidade de um erro tipo $I I$ seja menor do que ou igual a $\beta$ sempre que o verdadeiro valor de $\theta$ for maior do que ou igual a $\theta_{1}$.

Nesse caso, procede-se como a seguir. Considere a hipótese alternativa $H_{1}: \theta=\theta_{1}$. Daí, elabore um teste seqüencial para testar a hipótese $H_{0}: \theta=\theta_{0}$ contra $H_{1}$ tal que a probabilidade de um erro tipo $I$ (probabilidade de aceitar $H_{1}$ quando $H_{0}$ for verdadeira) seja $\alpha$ e a probabilidade de um erro tipo $I I$ (probabilidade de aceitar $H_{0}$ quando $H_{1}$ for verdadeira) seja $\beta$. Se o teste seqüencial possui a propriedade de que a probabilidade de um erro do tipo $I I$ seja menor do que ou igual a $\beta$ sempre que o verdadeiro valor de $\theta$ for maior do que $\theta_{1}$, então este teste seqüencial fornece uma solução satisfatória para o problema de testar a hipótese de que $\theta=\theta_{0}$ contra o conjunto das hipóteses alternativas de que $\theta>\theta_{0}$ (Wald, 1945).

O caso no qual os valores alternativos de $\theta$ são restritos aos valores menores do que $\theta_{0}$ é inteiramente análogo ao caso anterior, e não necessitam ser discutidos separadamente.

Wald (1945) ainda destaca que o processo seqüencial para testar $\theta=\theta_{0}$ contra 
$\theta>\theta_{0}$, é também adequado para testar a hipótese de que $\theta \leq \theta_{0}$, a qual fornece que a probabilidade de rejeitar a hipótese $\theta=\theta_{0}$ seja menor do que ou igual a $\alpha$ sempre que o verdadeiro valor de $\theta$ for menor do que ou igual a $\theta_{0}$.

\subsection{Plano de amostragem seqüencial para testar a média de uma distribuição binomial}

\subsubsection{Exposição do problema}

Uma distribuição binomial é utilizada quando o resultado das observações pode ser classificado em duas categorias. Por exemplo, ao decidir sobre a aplicação, ou não, de inseticida numa lavoura, sendo as unidades amostrais classificadas em danificadas ou não danificadas, ou ainda, infestadas ou não infestadas (Estefanel, 1977). Apresenta-se aqui o problema de testar a hipótese de que $p$ não excede um dado valor $p^{\prime}$ contra uma hipótese alternativa de que $p>p^{\prime}$. Nesse caso, Wald (1945) atribui o valor 0 para as unidades não infestadas e 1 para as unidades infestadas. Seja $p$ a proporção de unidades amostrais infestadas. Sendo $k$ o resultado da inspeção de uma unidade amostral escolhida ao acaso, podem-se tomar os valores 1 e 0 com probabilidades $p$ e $(1-p)$, respectivamente.

Caracteriza-se daí uma distribuição binomial cuja função de probabilidade é dada por

$$
f(k, m, p)=\left(\begin{array}{c}
m \\
k
\end{array}\right) p^{k}(1-p)^{m-k} \quad \text { para }(k=1,2, \ldots, m)
$$

sendo $(1-p)=q$ a proporção de unidades amostrais não infestadas e $m$ o número de unidades amostrais observadas.

\subsubsection{Riscos tolerados de tomar decisões erradas}

Segundo Estefanel (1977), um plano de amostragem pode levar a uma decisão errada, já que não inspeciona toda a população. Tal plano não pode garantir que uma decisão correta seja sempre tomada. A inspeção completa da população é muitas vezes impossível ou 
excessivamente dispendiosa, então podem-se tolerar riscos de tomar decisões erradas se isso permitir uma redução na quantidade de inspeções. Portanto, deve-se optar por um plano de amostragem seqüencial adequado, somente quando os riscos de tolerância tiverem sido fixados (Wald, 1945).

A finalidade de uma inspeção amostral é concluir sobre o grau de infestação da plantação, para então decidir se será, ou não, necessária a aplicação de inseticidas.

Deve-se, então, determinar o nível de infestação da plantação $p^{\prime}$ tal que a aplicação de inseticida seja necessária se $p>p^{\prime}$ e não seja necessária se $p \leq p^{\prime}$. Esse nível de infestação $p^{\prime}$ depende do preço do inseticida, do preço do produto colhido e do prejuízo causado pela praga (Estefanel, 1977).

Se $p=p^{\prime}$ o prejuízo causado pela praga é igual ao custo da aplicação do inseticida e a decisão de controlar ou não torna-se irrelevante. Se $p$ é pouco inferior a $p^{\prime}$, o custo da aplicação é também pouco inferior ao valor do prejuízo causado pela praga, nesse caso, a recomendação do controle não é considerada um erro de conseqüencias graves. Analogamente, se $p$ é levemente superior a $p^{\prime}$, o prejuízo causado pela praga é levemente superior ao custo da aplicação, então, a recomendação do controle nesse caso, não é um erro de conseqüencias graves (Estefanel, 1977).

Devem-se, então, determinar dois valores $p_{0}$ e $p_{1}$, onde $p_{0}<p<p_{1}$, tais que, não aconselhar a aplicação do inseticida seja um erro grave se, e somente se, $p \geq p_{1}$ e aconselhar a aplicação seja um erro grave se, e somente se, $p \leq p_{0}$.

Após $p_{0}$ e $p_{1}$ terem sido selecionados, o risco de tolerância pode ser fixado como a seguir: um plano de amostragem é necessário tal que a probabilidade de aceitar $H_{1}$ seja menor do que ou igual a $\alpha$ sempre que $p \leq p_{0}$, e a probabilidade de aceitar $H_{0}$ seja menor do que ou igual a $\beta$ sempre que $p \geq p_{1}$. Assim, os riscos tolerados são caracterizados por $p_{0}$, $p_{1}, \alpha$ e $\beta$, e esses valores devem ser escolhidos antes de se determinar o plano de amostragem adequado (Wald, 1945).

\subsubsection{Linhas de decisão do plano de amostragem seqüencial}

Considere as hipóteses $H_{0}: p=p_{0}$ e $H_{1}: p=p_{1}$ e ainda um teste seqüencial da razão de probabilidade para testar $H_{0}$ contra $H_{1}$. De acordo com Wald (1945), o teste seqüencial é dado como a seguir: em cada fase de inspeção, até a $m$-ésima observação, pode-se 
escrever

$$
\frac{p_{1 m}}{p_{0 m}}=\frac{p_{1}^{k_{m}}\left(1-p_{1}\right)^{m-k_{m}}}{p_{0}^{k_{m}}\left(1-p_{0}\right)^{m-k_{m}}}
$$

sendo $k_{m}$ o número de unidades amostrais infestadas, encontradas nas primeiras $m$ unidades inspecionadas.

Então, aceita-se $H_{1}$ se

$$
\frac{p_{1 m}}{p_{0 m}} \geq \frac{1-\beta}{\alpha}=A
$$

e aceita-se $H_{0}$ se

$$
\frac{p_{1 m}}{p_{0 m}} \leq \frac{\beta}{1-\alpha}=B
$$

Toma-se uma observação adicional se

$$
B=\frac{\beta}{1-\alpha}<\frac{p_{1 m}}{p_{0 m}}<\frac{1-\beta}{\alpha}=A .
$$

Podem-se escrever as inequações (25), (26) e (27) em uma forma diferente, tomando-se o logaritmo neperiano de ambos os lados verificam-se que essas inequações são equivalentes a

$$
\begin{aligned}
& k_{m} \geq \frac{\ln \left(\frac{1-\beta}{\alpha}\right)}{\ln \left(\frac{p_{1}}{p_{0}}\right)-\ln \left(\frac{1-p_{1}}{1-p_{0}}\right)}+m \frac{\ln \left(\frac{1-p_{0}}{1-p_{1}}\right)}{\ln \left(\frac{p_{1}}{p_{0}}\right)-\ln \left(\frac{1-p_{1}}{1-p_{0}}\right)} \\
& k_{m} \leq \frac{\ln \left(\frac{\beta}{1-\alpha}\right)}{\ln \left(\frac{p_{1}}{p_{0}}\right)-\ln \left(\frac{1-p_{0}}{1-p_{0}}\right)}+m \frac{\ln \left(\frac{p_{1}}{p_{0}}\right)-\ln \left(\frac{1-p_{1}}{1-p_{0}}\right)}{\ln }
\end{aligned}
$$

$\mathrm{e}$

$$
\begin{aligned}
\frac{\ln \left(\frac{\beta}{1-\alpha}\right)}{\ln \left(\frac{p_{1}}{p_{0}}\right)-\ln \left(\frac{1-p_{1}}{1-p_{0}}\right)}+m \frac{\ln \left(\frac{1-p_{0}}{1-p_{1}}\right)}{\ln \left(\frac{p_{1}}{p_{0}}\right)-\ln \left(\frac{1-p_{1}}{1-p_{0}}\right)} \\
<k_{m}<\frac{\ln \left(\frac{1-\beta}{\alpha}\right)}{\ln \left(\frac{p_{1}}{p_{0}}\right)-\ln \left(\frac{1-p_{1}}{1-p_{0}}\right)}+m \frac{\ln \left(\frac{1-p_{0}}{1-p_{1}}\right)}{\ln \left(\frac{p_{1}}{p_{0}}\right)-\ln \left(\frac{1-p_{1}}{1-p_{0}}\right)} .
\end{aligned}
$$


Considera-se que $q_{0}=1-p_{0}$ e $q_{1}=1-p_{1}$, os quais podem ser substituídos nas inequações (28), (29) e (30). Obtém-se, então,

$$
\begin{aligned}
& k_{m} \geq \frac{\ln \left(\frac{1-\beta}{\alpha}\right)}{\ln \left(\frac{p_{1}}{p_{0}}\right)-\ln \left(\frac{q_{1}}{q_{0}}\right)}+m \frac{\ln \left(\frac{q_{0}}{q_{1}}\right)}{\ln \left(\frac{p_{1}}{p_{0}}\right)-\ln \left(\frac{q_{1}}{q_{0}}\right)} \\
& k_{m} \leq \frac{\ln \left(\frac{\beta}{1-\alpha}\right)}{\ln \left(\frac{p_{1}}{p_{0}}\right)-\ln \left(\frac{q_{1}}{q_{0}}\right)}+m \frac{\ln \left(\frac{q_{0}}{q_{1}}\right)}{\ln \left(\frac{p_{1}}{p_{0}}\right)-\ln \left(\frac{q_{1}}{q_{0}}\right)}
\end{aligned}
$$

e

$$
\begin{aligned}
\frac{\ln \left(\frac{\beta}{1-\alpha}\right)}{\ln \left(\frac{p_{1}}{p_{0}}\right)-\ln \left(\frac{q_{1}}{q_{0}}\right)}+m \frac{\ln \left(\frac{q_{0}}{q_{1}}\right)}{\ln \left(\frac{p_{1}}{p_{0}}\right)-\ln \left(\frac{q_{1}}{q_{0}}\right)} \\
<k_{m}<\frac{\ln \left(\frac{1-\beta}{\alpha}\right)}{\ln \left(\frac{p_{1}}{p_{0}}\right)-\ln \left(\frac{q_{1}}{q_{0}}\right)}+m \frac{\ln \left(\frac{q_{0}}{q_{1}}\right)}{\ln \left(\frac{p_{1}}{p_{0}}\right)-\ln \left(\frac{q_{1}}{q_{0}}\right)} .
\end{aligned}
$$

Usando as inequações (31), (32) e (33) podem-se obter facilmente as linhas de decisão do plano de amostragem seqüencial que são representadas pelas equações a seguir

$$
S_{0}=\frac{\ln \left(\frac{\beta}{1-\alpha}\right)}{\ln \left(\frac{p_{1}}{p_{0}}\right)-\ln \left(\frac{q_{1}}{q_{0}}\right)}+m \frac{\ln \left(\frac{q_{0}}{q_{1}}\right)}{\ln \left(\frac{p_{1}}{p_{0}}\right)-\ln \left(\frac{q_{1}}{q_{0}}\right)}
$$

e

$$
S_{1}=\frac{\ln \left(\frac{1-\beta}{\alpha}\right)}{\ln \left(\frac{p_{1}}{p_{0}}\right)-\ln \left(\frac{q_{1}}{q_{0}}\right)}+m \frac{\ln \left(\frac{q_{0}}{q_{1}}\right)}{\ln \left(\frac{p_{1}}{p_{0}}\right)-\ln \left(\frac{q_{1}}{q_{0}}\right)}
$$

Usando-se a seguinte propriedade logarítmica

$$
\ln \left(\frac{a}{b}\right)=\ln (a)-\ln (b)
$$

verifica-se facilmente que 


$$
S_{0}=\frac{\ln \left(\frac{\beta}{1-\alpha}\right)}{\ln \left(\frac{p_{1} q_{0}}{p_{0} q_{1}}\right)}+m \frac{\ln \left(\frac{q_{0}}{q_{1}}\right)}{\ln \left(\frac{p_{1} q_{0}}{p_{0} q_{1}}\right)}
$$

$\mathrm{e}$

$$
S_{1}=\frac{\ln \left(\frac{1-\beta}{\alpha}\right)}{\ln \left(\frac{p_{1} q_{0}}{p_{0} q_{1}}\right)}+m \frac{\ln \left(\frac{q_{0}}{q_{1}}\right)}{\ln \left(\frac{p_{1} q_{0}}{p_{0} q_{1}}\right)}
$$

Aceita-se a hipótese $H_{0}$ se $k_{m} \leq S_{0}$; aceita-se a hipótese $H_{1}$ se $k_{m} \geq S_{1}$ e continua-se a amostragem se $S_{0}<k_{m}<S_{1}$.

Podem-se representar essas linhas, graficamente, como indicado na Figura 1. No eixo das abscissas, coloca-se o número de unidades amostrais inspecionadas $m$ e no eixo das ordenadas o número acumulado de unidades amostrais infestadas $k_{m}$ (Estefanel,1977).

O critério para o término da amostragem é dado a seguir:

i. Se o ponto $\left(m, k_{m}\right)$ estiver em $S_{0}$ ou abaixo dela, encerra-se a amostragem com aceitação de $H_{0}$; nesse caso não há necessidade de se optar pelo uso de inseticida.

ii. Se o ponto $\left(m, k_{m}\right)$ estiver em $S_{1}$ ou acima dela, encerra-se a amostragem com aceitação de $H_{1}$; nesse caso o uso de inseticida pode ser exigido.

iii. Se $\left(m, k_{m}\right)$ estiver entre $S_{0}$ e $S_{1}$, deve-se dar continuidade à amostragem até que $(i)$ ou (ii) ocorra. 


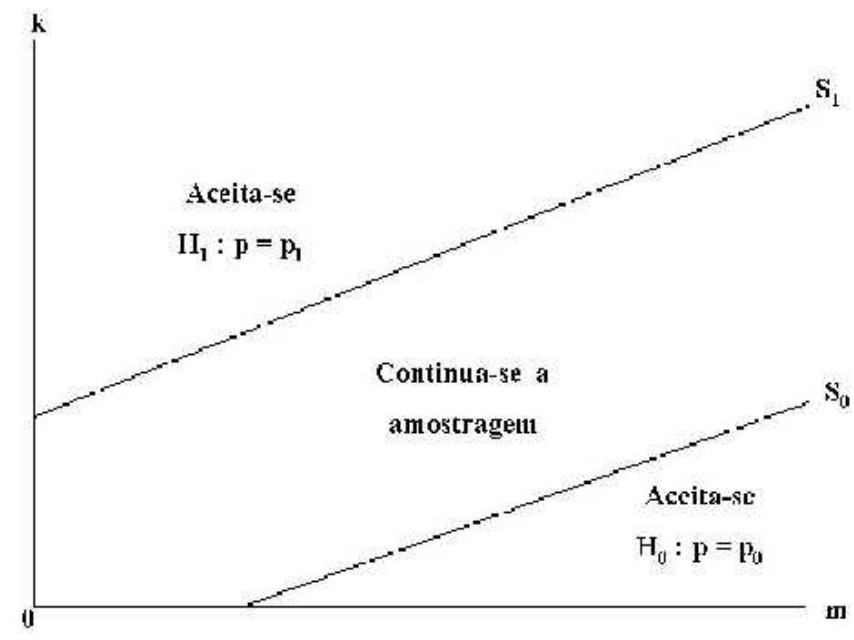

Figura 1 - Linhas de decisão do plano de amostragem seqüencial com base na distribuição binomial

\subsubsection{Curva característica operacional}

Como foi visto anteriormente, o teste seqüencial poderá satisfazer a condição de que a probabilidade de aceitação de $H_{0}$ seja menor do que ou igual a $\beta$ sempre que $p \geq p_{1}$ e de que a probabilidade de aceitação de $H_{1}$ seja menor do que ou igual a $\alpha$ sempre que $p \leq p_{0}$.

Wald (1945) considera $C O(p)$ como a probabilidade de aceitar $H_{0}$ para algum valor de $p$, desde que os valores de $\alpha$ e $\beta$ tenham sido pré-estabelecidos. $C O(p)$ é tida como uma função de $p$ e é chamada de Curva Característica Operacional, a qual fornece a probabilidade de tomar uma decisão correta para um determinado nível de infestação, e que pode ser representada graficamente como mostra a Figura 2. Sabe-se que a média de infestação $p$ pode tomar valores entre 0 e 1 , então, $C O(p)=1$ para $p=0$ e $C O(p)=0$ para $p=1$. Pode-se dizer, também, que $C O\left(p_{0}\right)=1-\alpha$ e que $C O\left(p_{1}\right)=\beta$.

Na curva característica operacional, WALD (1945) emprega uma variável auxiliar $h$ dependente de $p$. De acordo com a equação (16) pode-se obter uma aproximação para $C O(p)$ 


$$
C O(p) \sim 1-\frac{1-\left(\frac{\beta}{1-\alpha}\right)^{h}}{\left(\frac{1-\beta}{\alpha}\right)^{h}-\left(\frac{\beta}{1-\alpha}\right)^{h}}=\frac{\left(\frac{1-\beta}{\alpha}\right)^{h}-1}{\left(\frac{1-\beta}{\alpha}\right)^{h}-\left(\frac{\beta}{1-\alpha}\right)^{h}} .
$$

em que $h$ é a raiz não-nula da seguinte equação

$$
p\left(\frac{p_{1}}{p_{0}}\right)^{h}+(1-p)\left(\frac{1-p_{1}}{1-p_{0}}\right)^{h}=1
$$

Segundo Wald (1945), para se fazer o gráfico dessa curva, não é necessária a obtenção de $h$ através da equação (37), pode-se primeiramente, expressar $p$ como função de $h$,

$$
p=\frac{1-\left(\frac{1-p_{1}}{1-p_{0}}\right)^{h}}{\left(\frac{p_{1}}{p_{0}}\right)^{h}-\left(\frac{1-p_{1}}{1-p_{0}}\right)^{h}} .
$$

A partir daí, atribuem-se vários valores a $h$ e, então obtém-se os correspondentes valores de $p$ através de $(38)$ e de $C O(p)$ através de (36). O ponto $(p, C O(p))$ obtido será um ponto da curva característica operacional.

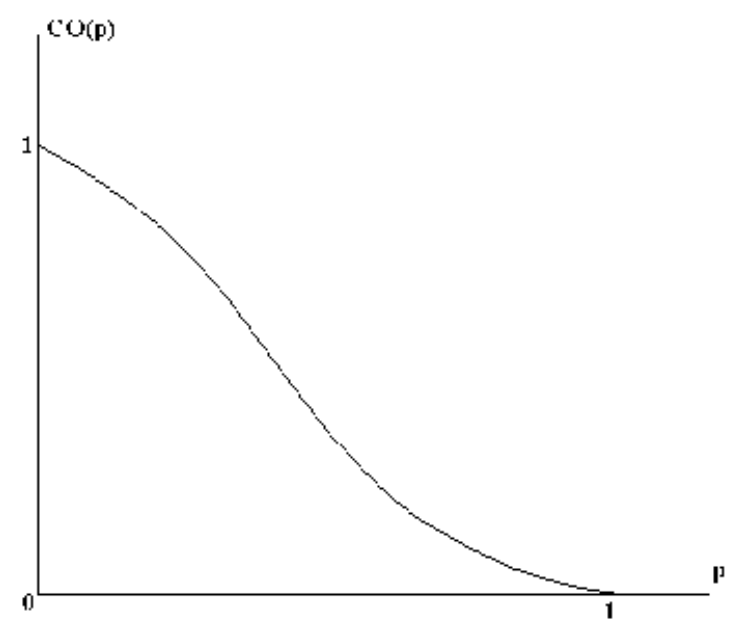

Figura 2 - Curva característica operacional para o plano de amostragem seqüencial com base na distribuição binomial 


\subsubsection{Curva do Número Médio de Unidades Amostrais}

O número de unidades amostrais exigido pela amostragem seqüencial não é pré-fixado, mas é uma variável aleatória, porque em cada estágio da amostragem a decisão de suspender o processo depende do resultado acumulado das observações anteriores (Estefanel,1977).

Seja $E_{p}(n)$ o número médio esperado de unidades amostrais para o teste seqüencial. De acordo com a expressão (23) obtém-se uma aproximação para $E_{p}(n)$

$$
E_{p}(n) \sim \frac{C O(p) \ln \left(\frac{\beta}{1-\alpha}\right)+(1-C O(p)) \ln \left(\frac{1-\beta}{\alpha}\right)}{p \ln \left(\frac{p_{1}}{p_{0}}\right)+(1-p) \ln \left(\frac{1-p_{1}}{1-p_{0}}\right)} .
$$

A curva do tamanho médio de unidades amostrais depende dos valores de $p$

e $(C O(p))$. Wald (1945) afirma que encontrar-se-á o máximo valor esperado de unidades amostrais quando $p$ estiver entre $p_{0}$ e $p_{1}$, e ainda que a curva será crescente quando o valor de $p$ crescer de 0 a $p_{0}$ e decrescente quando o valor de $p$ crescer de $p_{1}$ a 1 . Essa curva também pode ser representada graficamente como mostra a Figura 3.

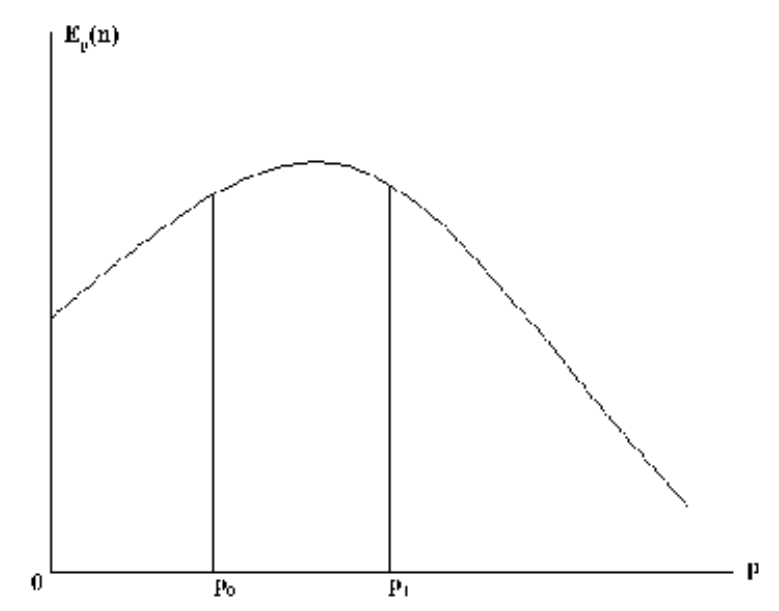

Figura 3 - Curva do número médio de unidades amostrais para o plano de amostragem seqüencial com base na distribuição binomial 


\section{MATERIAL E MÉTODOS}

\subsection{Material}

\subsubsection{Localização e datas de amostragens}

As áreas de estudo, Fazendas Bela Vista e Santa Maria, estão localizadas na região de Jaboticabal (21o18'45" $S ; 48 o 18^{\prime} 45^{\prime \prime} W ; 575 m$ ), interior do estado de São Paulo. Em cada propriedade foram escolhidas quatro parcelas (talhões), para fazer a coleta dos dados.

Na fazenda Bela Vista as amostragens foram realizadas entre 20/05/2003 e 10/10/2003 (que compreende desde o período vegetativo até o início do período de frutificação) nos talhões $6,7,9$ e 10, os quais foram chamados de $B V 6, B V 7, B V 9$ e $B V 10$, respectivamente, como mostra a Tabela 1. Nesta propriedade foram estudadas as variedades Tommy Atkins, Palmer, Haden e Keitt. 
Tabela 1. Datas de amostragens e variedades de manga em cada talhão da Fazenda Bela Vista, Jaboticabal, SP, 2003

\begin{tabular}{|c|c|c|c|}
\hline Talhão & $\begin{array}{l}\text { Estado fenológico } \\
\text { da planta }\end{array}$ & Datas de amostragens & Variedade \\
\hline \multirow[t]{4}{*}{ BV6 } & Vegetativo & $20 / 05 / 03,11 / 06 / 03$ & Tommy Atkins \\
\hline & & $21 / 06 / 03,09 / 07 / 03$ & \\
\hline & & $22 / 07 / 03,07 / 08 / 03$ & \\
\hline & Frutificação & $19 / 09 / 03$ & \\
\hline \multirow[t]{4}{*}{ BV7 } & Vegetativo & $21 / 05 / 03,11 / 06 / 03$ & Palmer \\
\hline & & $21 / 06 / 03,10 / 07 / 03$ & \\
\hline & & $23 / 07 / 03,08 / 08 / 03$ & \\
\hline & Frutificação & Não houve amostragem & \\
\hline \multirow[t]{5}{*}{ BV9 } & Vegetativo & $21 / 05 / 03,11 / 06 / 03$ & Haden \\
\hline & & $21 / 06 / 03,10 / 07 / 03$ & \\
\hline & & $23 / 07 / 03,08 / 08 / 03$ & \\
\hline & Frutificação & $27 / 08 / 03,18 / 09 / 03$ & \\
\hline & & $04 / 10 / 03$ & \\
\hline \multirow[t]{5}{*}{ BV10 } & Vegetativo & $21 / 05 / 03,11 / 06 / 03$ & Keitt \\
\hline & & $21 / 06 / 03,10 / 07 / 03$ & \\
\hline & & $23 / 07 / 03$ & \\
\hline & Frutificação & 08/08/03, 28/08/03 & \\
\hline & & $19 / 09 / 03,10 / 10 / 03$ & \\
\hline
\end{tabular}

Na fazenda Santa Maria foram coletadas amostras durante a fase vegetativa até o início da fase de frutificação, correspondendo ao período de 23/05/2003 até 19/09/2003, de acordo com a Tabela 2. Os quatro talhões desta propriedade foram chamados de $S M 1$, $S M 2, S M 3$ e $S M 4$, referente aos talhões 1, 2, 3 e 4, respectivamente, tendo como variedades estudadas, Tommy Atkins e Palmer. 
Tabela 2. Datas de amostragens e variedades de manga em cada talhão da Fazenda Santa Maria, Jaboticabal, SP, 2003

\begin{tabular}{cccc}
\hline Talhão & $\begin{array}{c}\text { Estado fenológico } \\
\text { da planta }\end{array}$ & Datas de amostragens & Variedade \\
\hline SM1 & Vegetativo & $23 / 05 / 03,11 / 06 / 03$ & Tommy Atkins \\
& & $25 / 06 / 03,12 / 07 / 03$ & \\
& & $29 / 07 / 03,15 / 08 / 03$ & \\
SM2 & Frutificação & $19 / 09 / 03$ & \\
& Vegetativo & $23 / 05 / 03,11 / 06 / 03$ & Tommy Atkins \\
& & $25 / 06 / 03,09 / 07 / 03$ & \\
& & $22 / 07 / 03$ & \\
SM3 & Vrutificação & $09 / 08 / 03,19 / 09 / 03$ & \\
& Vegetativo & $23 / 05 / 03,11 / 06 / 03$ & Tommy Atkins \\
& & $25 / 06 / 03,09 / 07 / 03$ & \\
& & $22 / 07 / 03$ & \\
& & $07 / 08 / 03,19 / 09 / 03$ & \\
& Frutificação & $23 / 05 / 03,12 / 06 / 03$ & \\
& Vegetativo & $25 / 06 / 03,11 / 07 / 03$ & \\
& & $24 / 07 / 03,09 / 08 / 03$ & \\
& & Não houve amostragem & \\
& & &
\end{tabular}

Com o interesse de manter uma uniformidade na coleta dos dados, os produtores resolveram adotar, aproximadamente, duas semanas como intervalo entre as datas de amostragens.

Em cada propriedade, a coleta dos dados foi realizada por um indivíduo previamente treinado denominado "pragueiro"(amostrador), munido de uma prancheta (ficha de inspeção), lápis ou caneta e uma lupa de aumento de 10 vezes. 


\subsection{Métodos}

\subsubsection{Monitoramento}

Em cada data de amostragem foi escolhida aleatoriamente uma planta localizada em um determinado talhão, não necessariamente na periferia. A amostragem foi iniciada por uma lateral desse talhão, escolhida ao acaso, e em amostragens subseqüentes iniciavam-se as observações por uma lateral oposta ou adjacente àquela tomada anteriormente. Isso foi feito com o objetivo de evitar que a amostra se tornasse viciada. Após a escolha da primeira planta, as demais foram amostradas caminhando-se em "zig-zag" pela área demarcada, sem a necessidade de contar passos. De acordo com o Comitê Gestor da Produção Integrada de Manga coordenado pela CATI-Regional Jaboticabal da Secretaria de Agricultura e Abastecimento de São Paulo, e posteriormente publicado por Souza Filho et al. (2004), o número de plantas amostradas em cada talhão obedeceu à proporção apresentada na Tabela 3.

Tabela 3. Número de plantas amostradas em função do tamanho dos talhões

\begin{tabular}{cc}
\hline Talhão de até 5 hectares & 10 plantas \\
Talhão de 6 a 10 hectares & 14 plantas \\
Talhão de 11 a 15 hectares & 18 plantas \\
Talhão acima de 15 hectares & Dividir em talhões menores
\end{tabular}

O espaçamento médio para a produção de manga é de $4 m \times 8 m$, ou seja, $32 m^{2}$ por planta. Isto equivale a dizer que um talhão de 5 hectares possui, aproximadamente, $\left(5 * 10.000\left(m^{2}\right)\right) / 32\left(m^{2} /\right.$ planta $)=50.000\left(m^{2}\right) / 32\left(m^{2} /\right.$ planta $)=1.562$ plantas.

Para o monitoramento, dividiu-se a copa da planta em quatro quadrantes imaginários. No período vegetativo, que vai da colheita até o florescimento, foi examinado um ramo em cada quadrante, localizado na parte interna (de preferência na parte baixa) da copa. No terceiro fluxo de crescimento de cada ramo, escolhia-se uma folha infestada, geralmente, a primeira folha com a presença da cochonilha que fosse vista pelo "pragueiro", para verificar se havia a forma jovem viva. Se houvesse, a folha era considerada danificada e anotava-se $R$ 
na ficha de inspeção, caso contrário, era considerada não danificada, e não era feita nenhuma anotação.

No período de frutificação, inspecionava-se além de um ramo, um fruto localizados na parte interna da copa (de preferência na parte baixa, que é a região mais sombreada) em cada quadrante. No fruto, caso a cochonilha fosse encontrada viva, tanto na forma jovem como adulta, esse era considerado danificado e então anotava-se $F$ na ficha, caso contrário, considerava-se não danificado, e não se faziam anotações. Se em um quadrante de uma determinada planta fossem encontrados um ramo e um fruto danificados, marcava-se $R / F$ na ficha de inspeção, e nada era anotado, caso fosse verificada a ausência de danos em ambos.

Referente à tabulação dos dados, se em um ou mais quadrantes de uma determinada planta fosse constatado que um ramo ou um fruto ou ambos estivessem danificados, a unidade amostral era considerada infestada, e então, anotava-se $1(U M)$ na tabela, caso contrário, anotava-se $0(Z E R O)$.

Não foi de interesse desse trabalho verificar o gradiente de infestação nas unidades amostrais. A divisão da planta em quadrantes foi feita para "obrigar" o "pragueiro" a dar uma volta completa na unidade amostral observada, facilitando assim o processo de amostragem e, também, evitando riscos de se verificar apenas uma parte da planta.

\subsubsection{Construção do plano de amostragem seqüencial}

É mais usual em estudos estatísticos, trabalhar com probabilidades iguais de incorrer em erros do tipo $I$ e do tipo $I I$. Como o objetivo deste trabalho consistiu em elaborar planos de amostragem seqüencial com níveis de erros diferentes, foram adotados dois valores para $\alpha$ (probabilidade de ocorrer um erro tipo $I$ ) e $\beta$ (probabilidade de ocorrer um erro tipo $I I)$, isto é, $\alpha=\beta=0,20$ e $\alpha=\beta=0,10$ que são os níveis mais utilizados em pesquisas agronômicas.

Segundo Allen et al. ${ }^{17}$ e Southwood ${ }^{18}$ mencionados por Farias et al. (2001b), a utilização do nível de erro igual a 0,20 leva ao tamanho da amostra necessário para a tomada de decisão mais viável, na prática, em um sistema de manejo de pragas. Já a utilização de $\alpha=\beta=0,10$, deve-se ao fato de ser um valor usual em planos seqüenciais usados por

\footnotetext{
${ }^{17}$ ALLEN, J.; GONZALEZ, D.; GOKHALE, D.V. Sequential sampling plans for the bollworn, Heliothis zea. Environmental Entomology, v.1, p.771-780, 1972.

${ }^{18}$ SOUTHWOOD, T.R.E. Ecological methods. New York: John Wiley \& Sons, 1978. 525p.
} 
entomologistas (Perecin \& Barbosa, 1995). Overholt et al. (1990) ainda destacam que o uso do nível de erro igual a 0,20 é comum para o manejo quanto à finalidade comercial, enquanto que o nível de 0,10 é comum para amostragens em pesquisas intensivas

No presente trabalho, foram elaborados seis planos de amostragem seqüencial, três com níveis de erros iguais a 0,20, adotados por Farias et al. (2001b), e outros três com níveis iguais a 0, 10 utilizados por Villacorta \& Tornero (1982), Wilson et al. (1983), Perecin \& Barbosa (1995) e Pereira et al. (2004). Foram feitos dois planos para a amostragem de ramos durante o período vegetativo, e quatro planos para serem utilizados durante o período de frutificação: dois para ramos e outros dois para frutos. Os fundamentos teóricos apresentados para a elaboração dos planos de amostragem seqüencial foram baseados em Wald (1945), por se tratar da base teórica do Teste Seqüencial da Razão de Probabilidades e, Estefanel (1977) e Farias et al. (2001b), que realizaram trabalhos práticos com o uso dessa teoria.

No caso da cochonilha-branca não é possível contar o número de indivíduos em uma determinada unidade amostral. Devido a isso, o método de amostragem utilizado foi o de presença-ausência, proposto por Wilson \& Room (1983).

Para a verificação do ajuste da distribuição binomial aos dados foi empregado o teste de Qui-quadrado $\left(\chi^{2}\right)$. As médias de plantas infestadas obtidas em cada data de amostragem, para cada um dos talhões, levando em consideração o período (vegetativo e de frutificação), foram comparadas com as médias típicas da distribuição binomial determinadas em cada talhão para os respectivos períodos. Com relação ao período de frutificação, a comparação foi feita separadamente para ramos e frutos.

Os níveis de segurança $\left(p_{0}\right)$ e de dano econômico $\left(p_{1}\right)$ foram adotados de acordo com o estado fenológico da planta, que no período de frutificação exige um cuidado maior quanto às médias de infestações. No período vegetativo as amostragens foram feitas em ramos, e foram adotados $p_{0}=0,35$ e $p_{1}=0,50$, já que nesse período a preocupação não é tão excessiva, devido à ausência de frutos. No período de frutificação deve-se ter um maior cuidado, então, nesse caso, foram utilizados $p_{0}=0,20$ e $p_{1}=0,30$ para as amostragens em ramos e, $p_{0}=0,05$ e $p_{1}=0,15$ para as amostragens em frutos.

Para o ajuste da distribuição binomial aos dados e para a determinação das linhas de decisão, da curva característica operacional e da curva do número médio de unidades amostrais, inclusive suas representações gráficas, foram utilizados os recursos do software estatístico $R$ versão 1.9.1, cujos comandos utilizados encontram-se em Anexos. 


\section{RESULTADOS E DISCUSSÃO}

\subsection{Ajuste da distribuição binomial aos dados de presença-ausência}

Ao aplicar o teste de $\chi^{2}$ para verificar o ajuste da distribuição binomial aos dados de presença-ausência da cochonilha-branca na cultura da manga, não foram satisfeitas todas as exigências do teste, assim como em Estefanel (1977), que não satisfez todas as exigências do teste ao verificar a aderência dos dados de contagens de lagartas da soja às distribuições teóricas: binomial, binomial negativa, normal e Poisson. Houve freqüências esperadas inferiores a 1 (um), mas isso deve-se ao fato de não terem sido encontradas cochonilhas vivas durante o período de frutificação para a amostragem em frutos e em ramos nos talhões BV6 e BV9, respectivamente. E também, durante o período de frutificação para a amostragem tanto em ramos quanto em frutos em todos os talhões da fazenda Santa Maria. Apenas nos talhões BV9 e SM4, ambos no período vegetativo, foram obtidas freqüências esperadas superiores a 5 (cinco).

O teste foi significativo em cinco casos para a amostragem em ramos, durante o período vegetativo nos talhões BV7, BV8, BV9 e SM3 e durante o período de frutificação no talhão BV10. Como na grande maioria dos casos o teste não foi significativo, pode-se afirmar que, as freqüências observadas combinaram com as freqüências esperadas, ou seja, que a distribuição binomial ajustou-se aos dados de presença-ausência da cochonilha-branca. 
Tabela 4. Ajuste da distribuição binomial aos dados para a amostragem realizada na Fazenda Bela Vista, onde $\chi^{2}$ calc. méd. é o valor de $\left(\chi^{2}\right.$ calculado na comparação entre as médias observadas em cada data de amostragem com a média da distribuição binomial) e $\chi^{2}$ tabela é o valor de $\left(\chi^{2}\right.$ tabelado com nível de probabilidade igual a 0,05)

\begin{tabular}{|c|c|c|c|c|c|c|c|}
\hline & & & $\begin{array}{c}\text { Média Típica } \\
\text { da Distribuição } \\
\text { Binomial }\end{array}$ & $\begin{array}{c}\text { Variância Típica } \\
\text { da Distribuição } \\
\text { Binomial }\end{array}$ & $\begin{array}{c}N^{o} \text { graus } \\
\text { de liberdade }\end{array}$ & $\chi^{2}$ calc. méd. & $\chi^{2}$ tabela \\
\hline & BV6 & Ramos & 3 & 2,357143 & 5 & 9,333333 & 11,070 \\
\hline & BV7 & Ramos & 2 & 1,714286 & 5 & 15 & 11,070 \\
\hline \multicolumn{8}{|l|}{ Período } \\
\hline \multirow[t]{7}{*}{ Vegetativo } & BV9 & Ramos & 5,833333 & 3,402778 & 5 & 23,114290 & 11,070 \\
\hline & BV10 & Ramos & 1,40 & 1,291111 & 4 & 13,714290 & 9,488 \\
\hline & & Ramos & 3 & 2,357143 & 1 & 0 & 3,841 \\
\hline & BV6 & & & & & & \\
\hline & & Frutos & 0 & 0 & 1 & 0 & 3,841 \\
\hline & & Ramos & NO & NO & NO & NO & NO \\
\hline & BV7 & & & & & & \\
\hline $\begin{array}{c}\text { Período } \\
\text { de }\end{array}$ & & Frutos & $\mathrm{NO}$ & NO & NO & NO & NO \\
\hline \multirow[t]{6}{*}{ Frutificação } & & Ramos & 0 & 0 & 2 & 0 & 5,991 \\
\hline & BV9 & & & & & & \\
\hline & & Frutos & 1,333333 & 1,206349 & 2 & 2 & 5,991 \\
\hline & & Ramos & 1,75 & 1,579861 & 3 & 21 & 7,815 \\
\hline & BV10 & & & & & & \\
\hline & & Frutos & 2,25 & 1,968750 & 3 & 3,888889 & 7,815 \\
\hline
\end{tabular}


Tabela 5. Ajuste da distribuição binomial aos dados para a amostragem realizada na Fazenda Santa Maria, onde $\chi^{2}$ calc. méd. é o valor de $\left(\chi^{2}\right.$ calculado na comparação entre as médias observadas em cada data de amostragem com a média da distribuição binomial) e $\chi^{2}$ tabela é o valor de $\left(\chi^{2}\right.$ tabelado com nível de probabilidade igual a 0,05)

\begin{tabular}{|c|c|c|c|c|c|c|c|}
\hline & & & $\begin{array}{c}\text { Média Típica } \\
\text { da Distribuição } \\
\text { Binomial }\end{array}$ & $\begin{array}{c}\text { Variância Típica } \\
\text { da Distribuição } \\
\text { Binomial }\end{array}$ & $\begin{array}{c}N^{o} \text { graus } \\
\text { de liberdade }\end{array}$ & $\chi^{2}$ calc. méd. & $\chi^{2}$ tabela \\
\hline & SM1 & Ramos & 2,666667 & 1,955556 & 5 & 9,5 & 11,070 \\
\hline & SM2 & Ramos & 1,8 & 1,476 & 4 & 7,111111 & 9,488 \\
\hline \multicolumn{8}{|l|}{ Período } \\
\hline \multirow[t]{7}{*}{ Vegetativo } & SM3 & Ramos & 1,6 & 1,417143 & 4 & 13,25 & 9,488 \\
\hline & SM4 & Ramos & 6,666667 & 3,492063 & 5 & 8,9 & 11,070 \\
\hline & & Ramos & 0 & 0 & 1 & 0 & 3,841 \\
\hline & SM1 & & & & & & \\
\hline & & Frutos & 0 & 0 & 1 & 0 & 3,841 \\
\hline & & Ramos & 0 & 0 & 1 & 0 & 3,841 \\
\hline & SM2 & & & & & & \\
\hline $\begin{array}{c}\text { Período } \\
\text { de }\end{array}$ & & Frutos & 0 & 0 & 1 & 0 & 3,841 \\
\hline \multirow[t]{6}{*}{ Frutificação } & & Ramos & 0 & 0 & 1 & 0 & 5,991 \\
\hline & SM3 & & & & & & \\
\hline & & Frutos & 0 & 0 & 1 & 2 & 5,991 \\
\hline & & Ramos & $\mathrm{NO}$ & NO & NO & NO & NO \\
\hline & SM4 & & & & & & \\
\hline & & Frutos & NO & NO & NO & NO & NO \\
\hline
\end{tabular}




\subsection{Planos de amostragem seqüencial com níveis de erros iguais a 0,20}

As linhas de decisão do plano de amostragem seqüencial para cada caso, foram obtidas através das expressões (34) e (35).

\subsubsection{Plano de amostragem seqüencial para ramos no período vegetativo}

Para a amostragem em ramos no período vegetativo, essas expressões encontram-se na Figura 4 e correspondem a $S_{0}=-2,239429+m * 0,423825$ até a qual aceita-se $H_{0}$, e $S_{1}=2,239429+m * 0,423825$ a partir da qual aceita-se $H_{1}$.

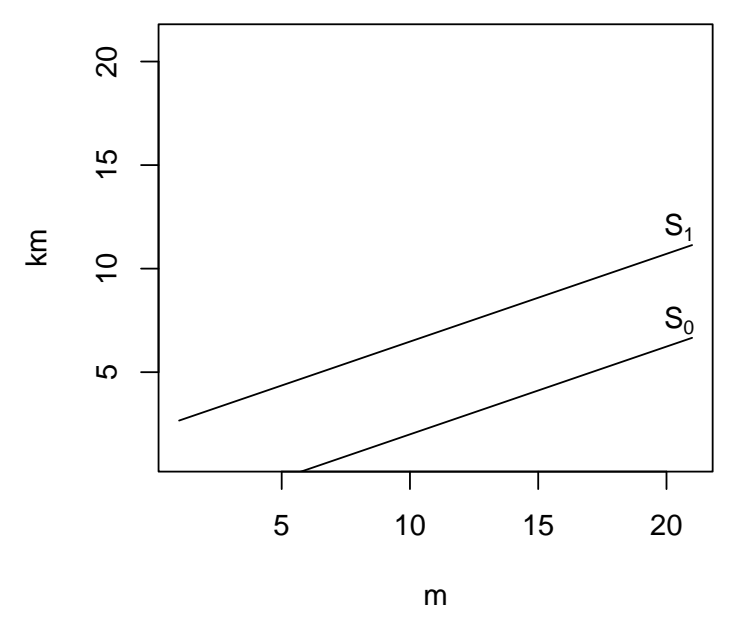

Figura 4 - Linhas de decisão do plano de amostragem seqüencial para ramos no período vegetativo, com níveis de erros iguais a 0,20 , onde m é o número de plantas observadas e km é o número de plantas infestadas

Através dos dados de $S_{0}$ e $S_{1}$ foi desenvolvida uma planilha para facilitar o trabalho de amostragem no campo (Tabela 6). O procedimento é feito da seguinte forma: na coluna da esquerda (limite inferior) foram anotados os pontos da linha $S_{0}$ e na coluna da direita (limite superior), os pontos da linha $S_{1}$. A tomada de decisão só pode ser feita a partir da $6^{a}$ unidade amostral. À medida que forem feitas as observações, deve-se anotar na coluna do meio (número acumulado de plantas infestadas) o número acumulado de unidades amostrais com a presença da cochonilha. Quando esse valor acumulado for igual ou inferior 
ao número da coluna da esquerda, salvo quando o limite inferior for igual a zero (0), pois por motivos óbvios, o valor acumulado só poderá ser igual ou superior a esse limite, suspende-se a amostragem e aceita-se $H_{0}$ não havendo a necessidade do controle, quando o valor acumulado for igual ou superior ao número da coluna da direita, suspende-se a amostragem e aceita-se $H_{1}$, nesse caso deve-se recomendar o controle. Se o número acumulado de plantas infestadas permanecer entre os limites inferior e superior, deve-se dar prosseguimento à amostragem até atingir o número máximo esperado de unidades amostrais que é de 21 plantas.

Se após ter atingido o número máximo esperado de plantas e nenhuma decisão tiver sido tomada, dever-se-á reduzir o período de amostragem pela metade, por exemplo, se o "pragueiro" estiver amostrando a cada duas semanas, esse deverá amostrar a cada sete (7) dias.

Tabela 6. Plano de amostragem seqüencial para o monitoramento da cochonilhabranca em ramos no período vegetativo, $\operatorname{com} \alpha=\beta=0,20$

\begin{tabular}{cccc}
\hline $\begin{array}{c}\text { Plantas } \\
\text { observadas }\end{array}$ & $\begin{array}{c}\text { Limite } \\
\text { inferior }\end{array}$ & $\begin{array}{c}N^{o} \text { acumulado de } \\
\text { plantas infestadas }\end{array}$ & $\begin{array}{c}\text { Limite } \\
\text { superior }\end{array}$ \\
\hline 1 & & & \\
2 & & & \\
3 & & & \\
4 & & & \\
5 & & 5 \\
6 & 0 & 5 \\
7 & 1 & 6 \\
8 & 1 & 6 \\
9 & 2 & 6 \\
10 & 2 & 7 \\
11 & 2 & 7 \\
12 & 3 & 8 \\
13 & 3 & 8 \\
14 & 4 & 9 \\
15 & 4 & 9 \\
16 & 5 & 11 \\
17 & 5 & 9 \\
18 & 5 & 10 \\
19 & 6 & \\
20 & 6 & \\
21 & 7 & \\
\hline
\end{tabular}


A curva característica operacional (Figura 5), apresenta a probabilidade de aceitação de $H_{0}$ para algum nível de infestação. Por exemplo, para uma proporção de aproximadamente $17 \%$ de unidades amostrais infestadas, o teste tem uma probabilidade igual a 0,9961 de aceitar $H_{0}$, ou seja, a probabilidade de recomendar o controle é igual a 0,0039.

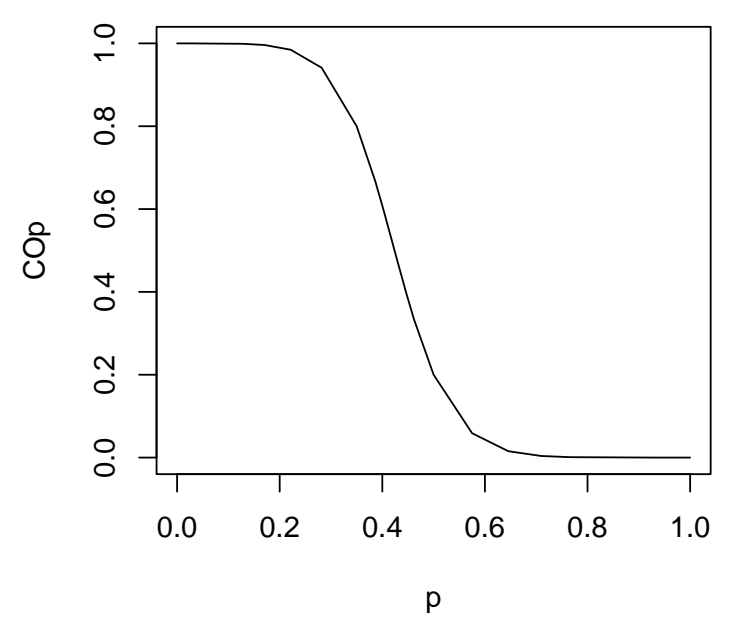

Figura 5 - Curva característica operacional do plano de amostragem seqüencial para ramos no período vegetativo, com níveis de erros iguais a 0,20 , onde p é proporção de plantas infestadas e COp a probabilidade de aceitar $H_{0}$

O máximo valor esperado de unidades amostrais (Figura 6)ocorre para uma infestação de aproximadamente 42\%, concordando com a afirmação de Wald (1945), isto é, que o máximo valor esperado de unidades amostrais para se tomar uma decisão ocorre quando $p_{0}<p<p_{1}$. 


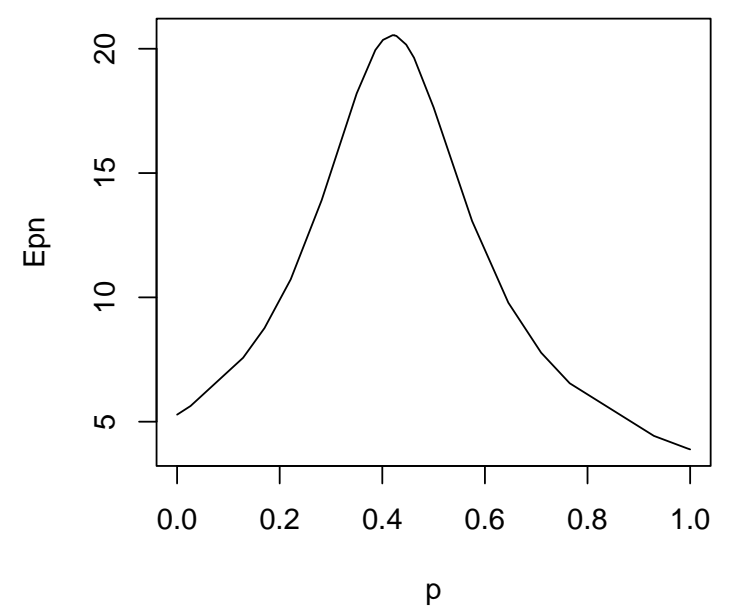

Figura 6 - Curva do número médio de unidades amostrais (Epn) do plano de amostragem seqüencial para ramos no período vegetativo, com níveis de erros iguais a 0,20 , onde p é a proporção de plantas infestadas

\subsubsection{Plano de amostragem seqüencial para ramos no período de frutificação}

Para ramos no período de frutificação, $S_{0}=-2,571991+m * 0,2477407$ até a qual $H_{0}$ deve ser aceita, e $S_{1}=2,571991+m * 0,2477407$ a partir da qual a aceitação de $H_{1}$ é necessária, encontram-se na Figura 7.

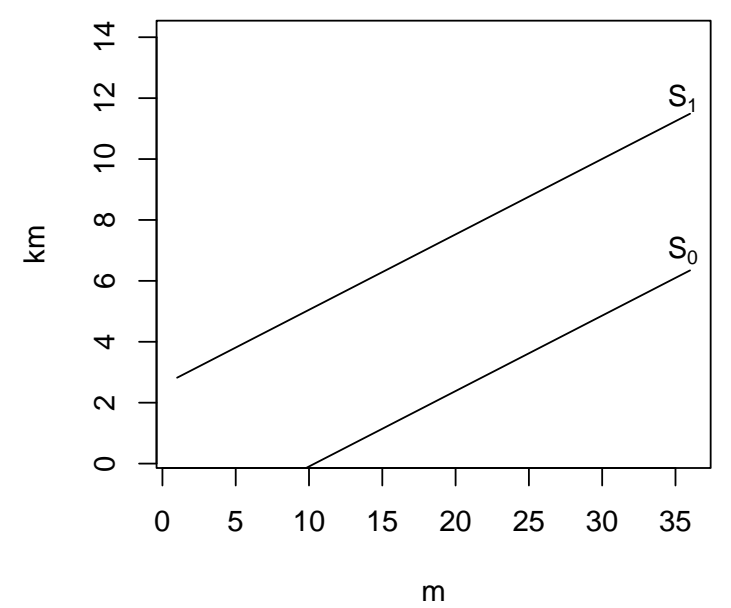

Figura 7 - Linhas de decisão do plano de amostragem seqüencial para ramos no período de frutificação, com níveis de erros iguais a 0,20 
Para o trabalho de amostragem no campo, foi construída uma planilha (Tabela 7) para a anotação do dados. O procedimento para a marcação dos limites inferior e superior e anotação dos dados observados, é o mesmo citado anteriormente para a amostragem em ramos no período vegetativo. A diferença é que agora, a tomada de decisão deve ser feita a

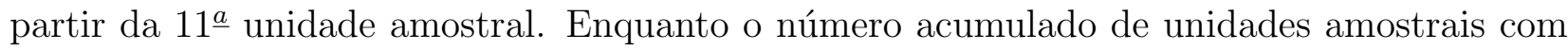
a presença da cochonilha permanecer na coluna do meio, deve-se continuar o processo de amostragem até atingir o valor máximo esperado de unidades amostrais que é de 36 plantas. Após ter alcançado o número máximo esperado de plantas e nenhuma decisão tiver sido tomada, dever-se-á então, reduzir o período de amostragem pela metade.

Tabela 7. Plano de amostragem seqüencial para o monitoramento da cochonilhabranca em ramos no período de frutificação, com $\alpha=\beta=0,20$. P.O. (Plantas observadas), L.I. (Limite inferior), $N^{o}$ Ac. (Número acumulado de plantas infestadas) e L.S. (Limite superior)

\begin{tabular}{|c|c|c|c|c|c|c|c|}
\hline P.O. & L.I. & $N^{o} \mathrm{Ac}$ & L.S. & P.O. & L.I. & $N^{o} \mathrm{Ac}$ & L.S. \\
\hline 1 & & & & 19 & 2 & & 7 \\
\hline 2 & & & & 20 & 2 & & 8 \\
\hline 3 & & & & 21 & 3 & & 8 \\
\hline 4 & & & & 22 & 3 & & 8 \\
\hline 5 & & & & 23 & 3 & & 8 \\
\hline 6 & & & & 24 & 3 & & 9 \\
\hline 7 & & & & 25 & 4 & & 9 \\
\hline 8 & & & & 26 & 4 & & 9 \\
\hline 9 & & & & 27 & 4 & & 9 \\
\hline 10 & & & & 28 & 4 & & 10 \\
\hline 11 & 0 & & 5 & 29 & 5 & & 10 \\
\hline 12 & 0 & & 6 & 30 & 5 & & 10 \\
\hline 13 & 1 & & 6 & 31 & 5 & & 10 \\
\hline 14 & 1 & & 6 & 32 & 5 & & 10 \\
\hline 15 & 1 & & 6 & 33 & 6 & & 11 \\
\hline 16 & 1 & & 7 & 34 & 6 & & 11 \\
\hline 17 & 2 & & 7 & 35 & 6 & & 11 \\
\hline 18 & 2 & & 7 & 36 & 6 & & 11 \\
\hline
\end{tabular}


A Figura 8 mostra a curva característica operacional, que apresenta a probabilidade de aceitar a hipótese $H_{0}$ para um nível de infestação qualquer.

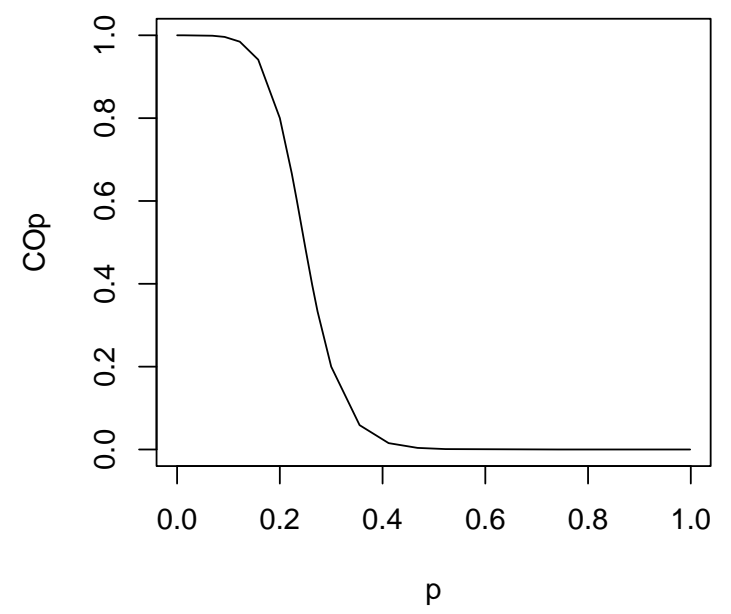

Figura 8 - Curva característica operacional do plano de amostragem seqüencial para ramos no período de frutificação, com níveis de erros iguais a 0,20

A curva do número esperado de unidades amostrais (Figura 9), mostra que para uma infestação de $74 \%$ de plantas com cochonilha, são necessárias 5 unidades amostrais para se tomar uma decisão, e para uma infestação de 12\%, são necessárias 20 plantas. Verifica-se novamente que o máximo valor esperado ocorre quando $p_{0}<p<p_{1}$, ou seja, para uma proporção de $25 \%$ de plantas infestadas, ocorre o número máximo esperado de unidades amostrais que é de 36 plantas. 


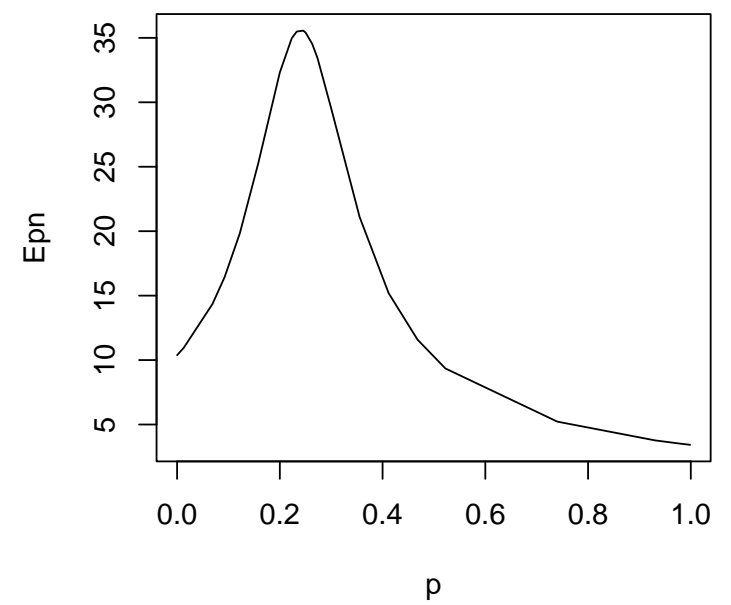

Figura 9 - Curva do número médio de unidades amostrais do plano de amostragem seqüencial para ramos no período de frutificação, com níveis de erros iguais a 0,20

\subsubsection{Plano de amostragem seqüencial para frutos}

Para a amostragem em frutos, as expressões das linhas de decisão podem ser visualizadas na Figura 10, essas correspondem a $S_{0}=-1,145851+m * 0,09193433$ até a qual deve-se aceitar $H_{0}$, e $S_{1}=1,145851+m * 0,09193433$ a partir da qual aceita-se $H_{1}$.

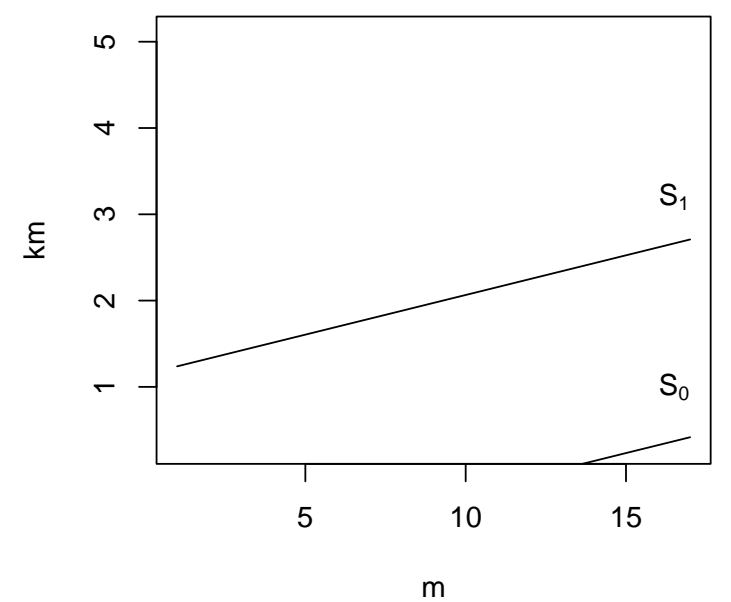

Figura 10 - Linhas de decisão do plano de amostragem seqüencial para frutos, com níveis de erros iguais a 0,20 
Através dos dados de $S_{0}$ e $S_{1}$ foi desenvolvida uma planilha para a anotação dos dados (Tabela 8), procedimento adotado, também, para os outros dois casos já mencionados. A forma de anotação dos limites inferior e superior, também já foi detalhada anteriormente para ramos no período vegetativo. Agora, a tomada de decisão deve ser feita a partir da $13^{a}$ unidade amostral. Enquanto o número acumulado de plantas infestadas permanecer na coluna do meio, deve-se dar prosseguimento à amostragem até atingir o valor máximo esperado de unidades amostrais que é de 17 plantas. Quando o número máximo esperado de plantas tiver sido alcançado, e nenhuma decisão for tomada, dever-se-á diminuir o tempo de amostragem pela metade.

Tabela 8. Plano de amostragem seqüencial para o monitoramento da cochonilhabranca em frutos, com $\alpha=\beta=0,20$

\begin{tabular}{cccc}
\hline $\begin{array}{c}\text { Plantas } \\
\text { observadas }\end{array}$ & $\begin{array}{c}\text { Limite } \\
\text { inferior }\end{array}$ & $\begin{array}{c}N^{o} \text { acumulado de } \\
\text { plantas infestadas }\end{array}$ & $\begin{array}{c}\text { Limite } \\
\text { superior }\end{array}$ \\
\hline 1 & & & \\
2 & & & \\
3 & & & \\
4 & & & \\
5 & & & \\
6 & & & \\
7 & & & \\
8 & & & \\
9 & & & \\
10 & & & \\
11 & & & 3 \\
12 & & & 3 \\
13 & 0 & & 3 \\
14 & 0 & & \\
15 & 0 & & \\
16 & 0 & & \\
17 & 0 & & \\
\hline
\end{tabular}


Através da curva característica operacional Figura 11), pode-se tomar como exemplo, uma proporção de $29 \%$ de plantas infestadas, nesse caso a probabilidade de aceitar $H_{0}$ é de 0,0154 , isto é, a probabilidade de recomendar o controle é de 0,9846 .

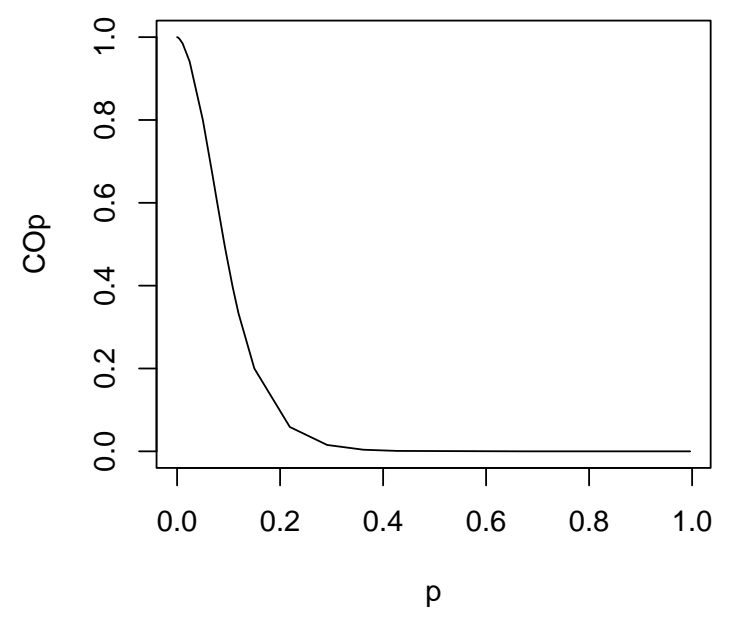

Figura 11 - Curva característica operacional do plano de amostragem seqüencial para frutos, com níveis de erros iguais a 0,20

Pode-se verificar através da curva do número esperado de unidades amostrais (Figura 12), que o máximo valor esperado de observações (17 plantas) ocorre para uma proporção de $7 \%$ de unidades amostrais infestadas. Como exemplo, pode-se verificar, também, que para uma proporção de $43 \%$ de plantas com a presença da cochonilha, são necessárias apenas 3 unidades amostrais para se tomar uma decisão, enquanto que para uma infestação de $1 \%$, são necessárias 14 observações. 


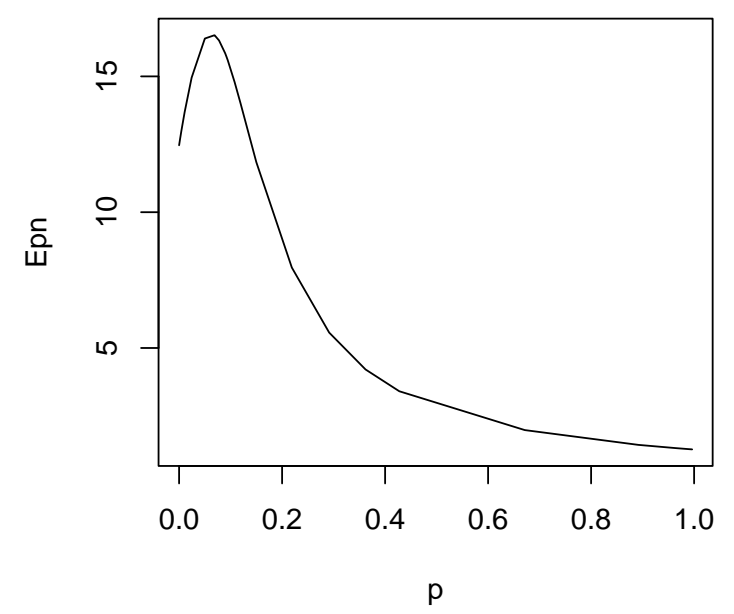

Figura 12 - Curva do número médio de unidades amostrais do plano de amostragem seqüencial para frutos, com níveis de erros iguais a 0,20

\subsection{Planos de amostragem seqüencial com níveis de erros iguais a 0,10}

Assim como nos planos com níveis de erros iguais a 0,20, as linhas de decisão do plano de amostragem seqüencial para cada caso, foram obtidas através das expressões (34) e (35).

\subsubsection{Plano de amostragem seqüencial para ramos no período vegetativo}

Na Figura 13 encontram-se as expressões das linhas de decisão do plano de amostragem seqüencial para ramos no período vegetativo com níveis de erros iguais a 0,10. Essas expressões são $S_{0}=-3,549411+m * 0,423825$ e $S_{1}=3,549411+m * 0,423825$. Quando o ponto ordenado $\left(m, k_{m}\right)$ estiver sobre $S_{0}$ ou abaixo dela, deve-se aceitar a hipótese $H_{0}$ e quando esse ponto estiver sobre $S_{1}$ ou acima dela, a aceitação da hipótese $H_{1}$ deverá ser exigida. 


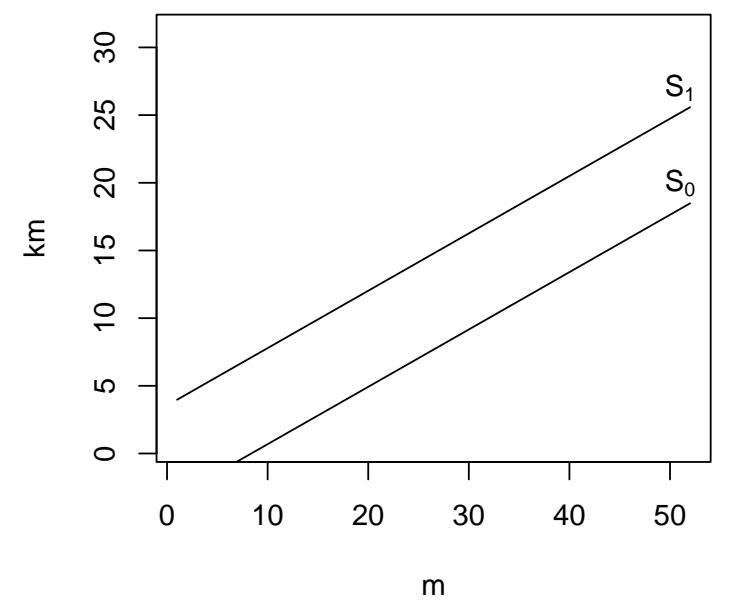

Figura 13 - Linhas de decisão do plano de amostragem seqüencial para ramos no período vegetativo, com níveis de erros iguais a 0,10

A Tabela 9 foi elaborada para facilitar o trabalho de inspeção da cochonilha. O procedimento para marcação dos limites inferior e superior e, também, para anotação dos dados coletados é o mesmo adotado para ramos no período vegetativo, quando foram considerados níveis de erros iguais a 0,20. A tomada de decisão para este caso, pode ser feita a partir da $9^{\underline{a}}$ observação. Se o número acumulado permanecer entre os limites inferior e superior, deve-se continuar o processo de amostragem até o número máximo esperado de observações que é de 52 plantas. 
Tabela 9. Plano de amostragem seqüencial para o monitoramento da cochonilhabranca em ramos no período vegetativo, $\operatorname{com} \alpha=\beta=0,10$. P.O. (Plantas observadas), L.I. (Limite inferior), $N^{o}$ Ac. (Número acumulado de plantas infestadas) e L.S. (Limite superior)

\begin{tabular}{|c|c|c|c|c|c|c|c|}
\hline P.O. & L.I. & $N^{o}$ Ac. & L.S. & P.O. & L.I. & $N^{o}$ Ac. & L.S. \\
\hline 1 & & & & 27 & 8 & & 15 \\
\hline 2 & & & & 28 & 8 & & 15 \\
\hline 3 & & & & 29 & 9 & & 16 \\
\hline 4 & & & & 30 & 9 & & 16 \\
\hline 5 & & & & 31 & 10 & & 17 \\
\hline 6 & & & & 32 & 10 & & 17 \\
\hline 7 & & & & 33 & 10 & & 18 \\
\hline 8 & & & & 34 & 11 & & 18 \\
\hline 9 & 0 & & 7 & 35 & 11 & & 18 \\
\hline 10 & 1 & & 8 & 36 & 12 & & 19 \\
\hline 11 & 1 & & 8 & 37 & 12 & & 19 \\
\hline 12 & 2 & & 9 & 38 & 13 & & 20 \\
\hline 13 & 2 & & 9 & 39 & 13 & & 20 \\
\hline 14 & 2 & & 9 & 40 & 13 & & 21 \\
\hline 15 & 3 & & 10 & 41 & 14 & & 21 \\
\hline 16 & 3 & & 10 & 42 & 14 & & 21 \\
\hline 17 & 4 & & 11 & 43 & 15 & & 22 \\
\hline 18 & 4 & & 11 & 44 & 15 & & 22 \\
\hline 19 & 5 & & 12 & 45 & 16 & & 23 \\
\hline 20 & 5 & & 12 & 46 & 16 & & 23 \\
\hline 21 & 5 & & 12 & 47 & 16 & & 23 \\
\hline 22 & 6 & & 13 & 48 & 17 & & 24 \\
\hline 23 & 6 & & 13 & 49 & 17 & & 24 \\
\hline 24 & 7 & & 14 & 50 & 18 & & 25 \\
\hline 25 & 7 & & 14 & 51 & 18 & & 25 \\
\hline 26 & 7 & & 15 & 52 & 18 & & 26 \\
\hline
\end{tabular}

Pela curva característica operacional (Figura 14), pode-se verificar que para uma proporção de plantas infestadas igual a 50\%, há uma probabilidade igual a 0,10 de que a hipótese $H_{0}$ seja aceita, isto é, a probabilidade de se recomendar o controle (aceitar $H_{1}$ ) é de 0,90. E para uma infestação de 35\%, a probabilidade de aceitar $H_{0}$ é igual a 0,90 e a probabilidade de aceitar $H_{1}$ é igual a 0,10 . 


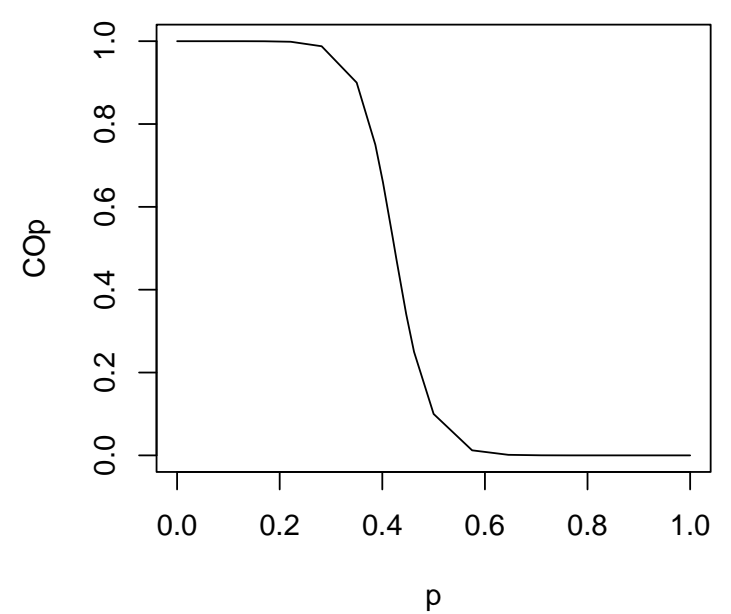

Figura 14 - Curva característica operacional do plano de amostragem seqüencial para ramos no período vegetativo, com níveis de erros iguais a 0,10

De acordo com a curva do número esperado de unidades amostrais observadas (Figura 15), verifica-se que o máximo valor esperado de observações é de 52 plantas e isso ocorre quando há uma proporção de $42 \%$ de plantas com a presença da cochonilha.

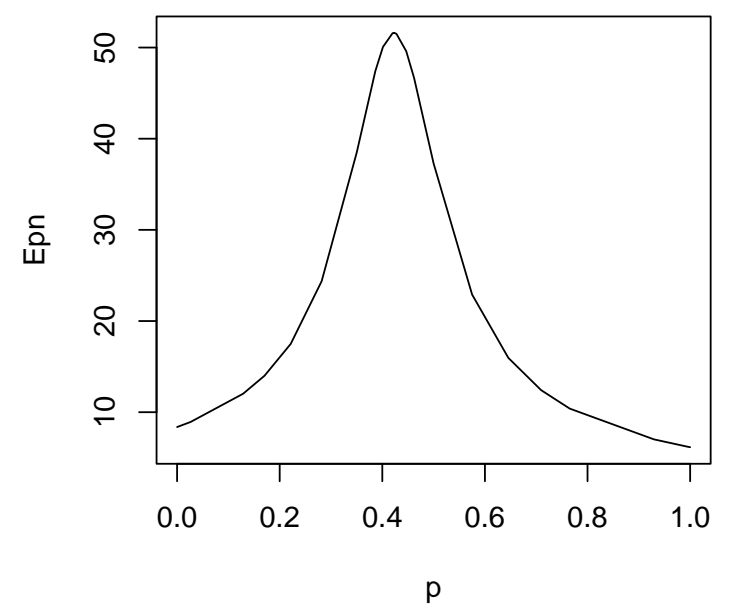

Figura 15 - Curva do número médio de unidades amostrais do plano de amostragem seqüencial para ramos no período vegetativo, com níveis de erros iguais a 0,10 


\subsubsection{Plano de amostragem seqüencial para ramos no período de frutificação}

Na Figura 16 pode-se verificar graficamente que, as linhas de decisão do plano de amostragem seqüencial são representadas pelas expressões $S_{0}=-4,07651+m * 0,2477407$ até a qual a aceitação da hipótese $H_{0}$ deve ser exigida e $S_{1}=4,07651+m * 0,2477407$ a partir da qual a aceitação da hipótese $H_{1}$ torna-se necessária.

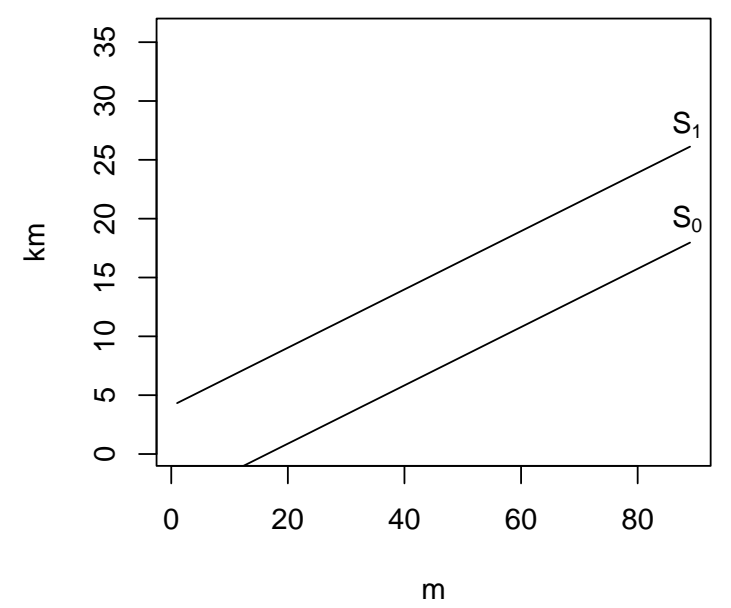

Figura 16 - Linhas de decisão do plano de amostragem seqüencial para ramos no período de frutificação, com níveis de erros iguais a 0,10

Segue o mesmo procedimento mencionado anteriormente, tanto para marcação dos limites inferior e superior quanto para anotação dos dados. A tomada de decisão para esse caso pode ser feita a partir da $17^{\underline{a}}$ observação. A aceitação da hipótese $H_{0}$ pode ser feita quando o número acumulado de plantas com a presença da cochonilha for igual ou inferior ao valor do limite inferior (L.I.), e deve-se aceitar a hipótese $H_{1}$ quando esse número acumulado for maior do que ou igual ao limite superior (L.S.). O máximo valor esperado de unidades amostrais para esse plano de amostragem é de 89 plantas, e enquanto o número acumulado de plantas infestadas permanecer entre L.I. e L.S., deve-se continuar o processo de amostragem até atingir as 89 observações, a partir da qual o período de coleta dos dados será reduzido pela metade. 
Tabela 10. Plano de amostragem seqüencial para o monitoramento da cochonilhabranca em ramos no período de frutificação, com $\alpha=\beta=0,10$. P.O. (Plantas observadas), L.I. (Limite inferior), $N^{o}$ Ac. (Número acumulado de plantas infestadas) e L.S. (Limite superior)

\begin{tabular}{|c|c|c|c|c|c|c|c|}
\hline P.O. & L.I. & $N^{o}$ Ac. & L.S. & P.O. & L.I. & $N^{o}$ Ac. & L.S. \\
\hline 1 & & & & 46 & 7 & & 15 \\
\hline 2 & & & & 47 & 8 & & 16 \\
\hline 3 & & & & 48 & 8 & & 16 \\
\hline 4 & & & & 49 & 8 & & 16 \\
\hline 5 & & & & 50 & 8 & & 16 \\
\hline 6 & & & & 51 & 9 & & 17 \\
\hline 7 & & & & 52 & 9 & & 17 \\
\hline 8 & & & & 53 & 9 & & 17 \\
\hline 9 & & & & 54 & 9 & & 17 \\
\hline 10 & & & & 55 & 10 & & 18 \\
\hline 11 & & & & 56 & 10 & & 18 \\
\hline 12 & & & & 57 & 10 & & 18 \\
\hline 13 & & & & 58 & 10 & & 18 \\
\hline 14 & & & & 59 & 11 & & 19 \\
\hline 15 & & & & 60 & 11 & & 19 \\
\hline 16 & & & & 61 & 11 & & 19 \\
\hline 17 & 0 & & 8 & 62 & 11 & & 19 \\
\hline 18 & 0 & & 9 & 63 & 12 & & 20 \\
\hline 19 & 1 & & 9 & 64 & 12 & & 20 \\
\hline 20 & 1 & & 9 & 65 & 12 & & 20 \\
\hline 21 & 1 & & 9 & 66 & 12 & & 20 \\
\hline 22 & 1 & & 10 & 67 & 13 & & 21 \\
\hline 23 & 2 & & 10 & 68 & 13 & & 21 \\
\hline 24 & 2 & & 10 & 69 & 13 & & 21 \\
\hline 25 & 2 & & 10 & 70 & 13 & & 21 \\
\hline 26 & 2 & & 11 & 71 & 14 & & 22 \\
\hline 27 & 3 & & 11 & 72 & 14 & & 22 \\
\hline 28 & 3 & & 11 & 73 & 14 & & 22 \\
\hline 29 & 3 & & 11 & 74 & 14 & & 22 \\
\hline 30 & 3 & & 12 & 75 & 15 & & 23 \\
\hline 31 & 4 & & 12 & 76 & 15 & & 23 \\
\hline 32 & 4 & & 12 & 77 & 15 & & 23 \\
\hline 33 & 4 & & 12 & 78 & 15 & & 23 \\
\hline 34 & 4 & & 12 & 79 & 15 & & 24 \\
\hline 35 & 5 & & 13 & 80 & 16 & & 24 \\
\hline 36 & 5 & & 13 & 81 & 16 & & 24 \\
\hline 37 & 5 & & 13 & 82 & 16 & & 24 \\
\hline 38 & 5 & & 13 & 83 & 16 & & 25 \\
\hline 39 & 6 & & 14 & 84 & 17 & & 25 \\
\hline 40 & 6 & & 14 & 85 & 17 & & 25 \\
\hline 41 & 6 & & 14 & 86 & 17 & & 25 \\
\hline 42 & 6 & & 14 & 87 & 17 & & 26 \\
\hline 43 & 7 & & 15 & 88 & 18 & & 26 \\
\hline 44 & 7 & & 15 & 89 & 18 & & 26 \\
\hline 45 & 7 & & 15 & $\ldots$ & $\ldots$ & & $\ldots$ \\
\hline
\end{tabular}

Através da curva característica operacional (Figura 17), determina-se a probabilidade de aceitar $H_{0}$ para um determinado nível de infestação. 


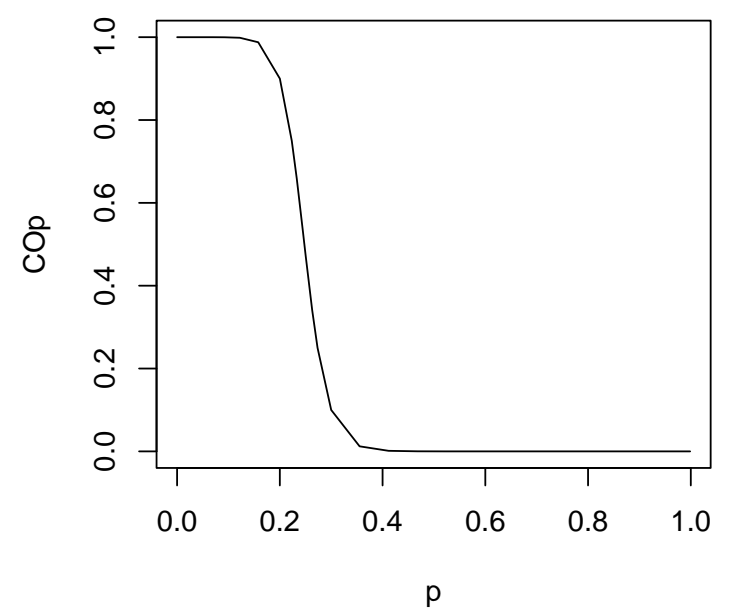

Figura 17 - Curva característica operacional do plano de amostragem seqüencial para ramos no período de frutificação, com níveis de erros iguais a 0,10

O máximo valor esperado de observações ocorre para uma proporção igual a $24 \%$ de plantas infestadas, e isso pode ser visto através da Figura 18 (Curva do Número Médio de Unidades Amostrais).

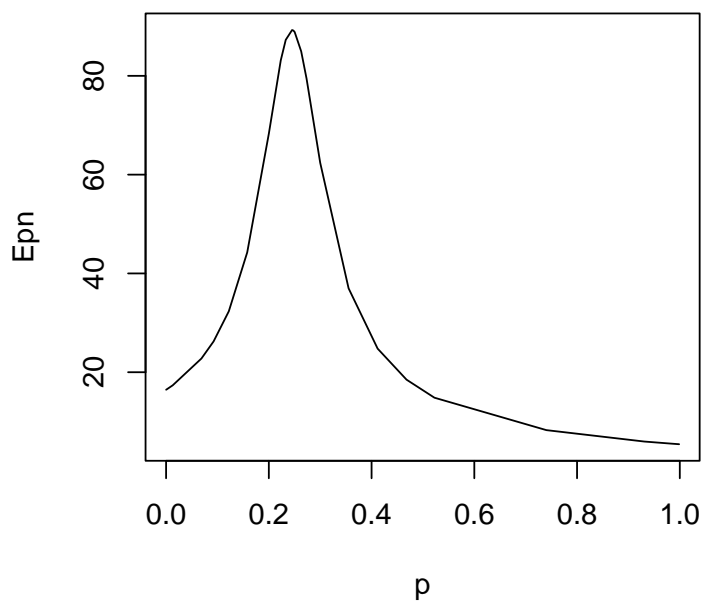

Figura 18 - Curva do número médio de unidades amostrais do plano de amostragem seqüencial para ramos no período de frutificação, com níveis de erros iguais a 0,10 


\subsubsection{Plano de amostragem seqüencial para frutos}

$$
S_{0}=-1,816131+m * 0,09193433 \text { até a qual aceita-se a hipótese } H_{0} \text { e } S_{1}=
$$
$1,816131+m * 0,09193433$ a partir da qual a aceitação da hipótese $H_{1}$ deve ser exigida, são as expressões das linhas de decisão, e essas podem ser observadas na Figura 19.

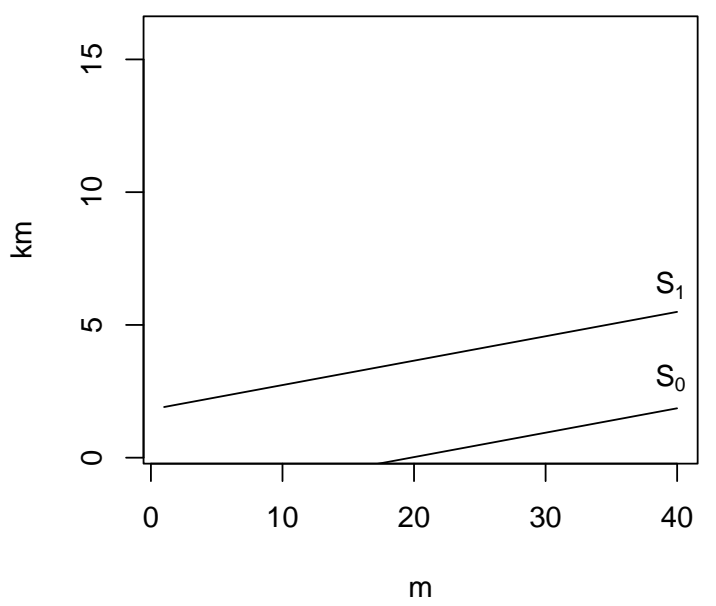

Figura 19 - Linhas de decisão do plano de amostragem seqüencial para frutos, com níveis de erros iguais a 0,10

Deve-se adotar o mesmo procedimento anteriormente mencionado (marcação dos limites, anotação dos dados e tomada de decisão). Agora, pode-se tomar uma decisão a partir da $20^{\underline{a}}$ planta observada. Se o número acumulado de plantas infestadas permanecer entre os limites inferior e superior, continua-se o processo de amostragem até o valor máximo esperado de observações que é de 40 plantas, quando deve-se reduzir o tempo de amostragem ao meio. Por exemplo, se o "pragueiro" estiver amostrando a cada duas semanas, esse deverá diminuir o período para 7 (sete) dias e se estiver coletando os dados toda semana, essa coleta deverá acontecer a cada 3 (três) ou 4 (quatro) dias. 
Tabela 11. Plano de amostragem seqüencial para o monitoramento da cochonilhabranca em frutos, com $\alpha=\beta=0,10$. P.O. (Plantas observadas), L.I. (Limite inferior), $N^{o}$ Ac. (Número acumulado de plantas infestadas) e L.S. (Limite superior)

\begin{tabular}{|c|c|c|c|c|c|c|c|}
\hline P.O. & L.I. & $N^{o}$ Ac. & L.S. & P.O. & L.I. & $N^{o} \mathrm{Ac}$ & L.S. \\
\hline 1 & & & & 21 & 0 & & 4 \\
\hline 2 & & & & 22 & 0 & & 4 \\
\hline 3 & & & & 23 & 0 & & 4 \\
\hline 4 & & & & 24 & 0 & & 4 \\
\hline 5 & & & & 25 & 0 & & 4 \\
\hline 6 & & & & 26 & 1 & & 4 \\
\hline 7 & & & & 27 & 1 & & 4 \\
\hline 8 & & & & 28 & 1 & & 4 \\
\hline 9 & & & & 29 & 1 & & 4 \\
\hline 10 & & & & 30 & 1 & & 5 \\
\hline 11 & & & & 31 & 1 & & 5 \\
\hline 12 & & & & 32 & 1 & & 5 \\
\hline 13 & & & & 33 & 1 & & 5 \\
\hline 14 & & & & 34 & 1 & & 5 \\
\hline 15 & & & & 35 & 1 & & 5 \\
\hline 16 & & & & 36 & 1 & & 5 \\
\hline 17 & & & & 37 & 2 & & 5 \\
\hline 18 & & & & 38 & 2 & & 5 \\
\hline 19 & & & & 39 & 2 & & 5 \\
\hline 20 & 0 & & 4 & 40 & 2 & & 5 \\
\hline
\end{tabular}

A curva Característica Operacional Figura 20, indica que, para uma proporção de plantas infestadas igual a 5\%, há uma probabilidade igual a 0,90 de aceitar $H_{0}$, isto é, há uma probabilidade igual a 0,10 de que o controle seja recomendado. E para uma infestação de $15 \%$, a decisão de não controlar (aceitar $H_{0}$ ) ocorre com uma probabilidade igual a 0,10 , isto é, a probabilidade de aceitar $H_{1}$ é de 0,90 . 


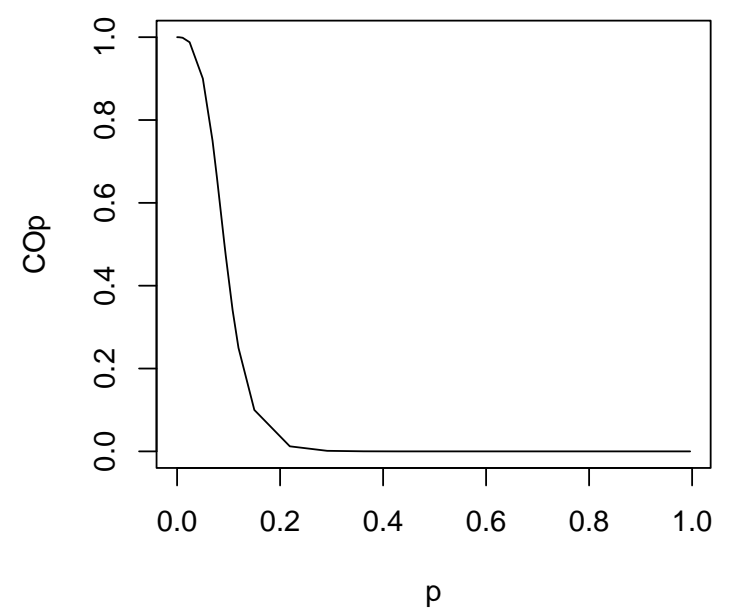

Figura 20 - Curva característica operacional do plano de amostragem seqüencial para frutos, com níveis de erros iguais a 0,10

O número máximo esperado de unidades amostrais para esse plano de amostragem seqüencial é de 40 plantas, e segundo a curva do número médio de unidades amostrais (Figura 21), isso acontece para uma proporção de plantas infestadas igual a $8 \%$.

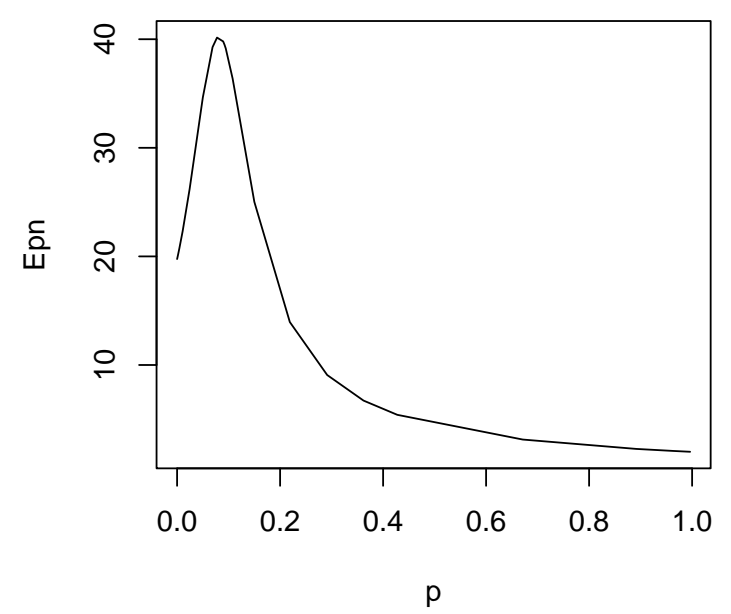

Figura 21 - Curva do número médio de unidades amostrais do plano de amostragem seqüencial para frutos, com níveis de erros iguais a 0,10 


\section{CONCLUSÕES}

Face as metodologias empregadas nesse trabalho, os dados estudados e os resultados obtidos, pode-se concluir que:

i) a distribuição binomial ajustou-se aos dados de presença-ausência da cochonilha-branca;

ii) devem-se utilizar os planos de amostragem com nível de erro igual a 0,20, pois além de fornecerem uma boa precisão na análise dos dados, esses planos fornecem, também, tamanhos esperados de amostras menores, reduzindo assim, os custos de amostragem;

iii) devido às diferenças nas condições climáticas de uma região para outra, a aplicação desses planos deve ser feita apenas em propriedades localizadas nos municípios da região noroeste do estado de São Paulo;

iv) esses planos de amostragem devem ser aplicados somente em talhões de até 5 hectares, a partir do qual estes deverão ser subdivididos em talhões menores.

A proposta original desse trabalho foi cumprida, fornecendo planos de amostragem seqüencial com níveis de erros diferentes, construídos com base na teoria do Teste Seqüencial da Razão de Probabilidades de Wald. 
ANEXOS 
ANEXO A - Aplicação do teste de Qui-quadrado.

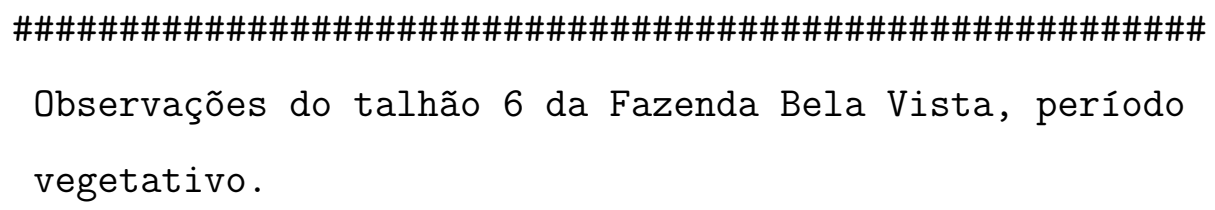


$\operatorname{var} 4$

\# Quinta data de amostragem (22/07/2003).

bv6veg $5<-c(1,1,0,0,0,1,0,0,0,1,0,0,0,1)$

med5<-mean (bv6veg5)

med5

$\operatorname{var} 5<-\operatorname{var}($ bv6veg5)

$\operatorname{var} 5$

\# Sexta data de amostragem (07/08/2003).

bv6veg6<-c $(1,0,0,0,0,0,1,0,0,0,0,1,0,1)$

med6<-mean (bv6veg6)

med6

$\operatorname{var} 6<-\operatorname{var}($ bv6veg6)

$\operatorname{var} 6$

\# Média geral e média das variâncias.

$\mathrm{p}<-(\operatorname{med} 1+\operatorname{med} 2+\operatorname{med} 3+\operatorname{med} 4+\operatorname{med} 5+\operatorname{med} 6) / 6$

p

medvar<- $(\operatorname{var} 1+\operatorname{var} 2+\operatorname{var} 3+\operatorname{var} 4+\operatorname{var} 5+\operatorname{var} 6) / 6$

medvar

\# Média e variância para a distribuição binomial.

$\mathrm{np}<-14 * \mathrm{p}$

$\mathrm{np}$

$\mathrm{npq}<-14 * \mathrm{p} *(1-\mathrm{p})$

$\mathrm{npq}$

\# Aplicação do teste de qui-quadrado para verificar a eficiência do ajuste da distribuição binomial aos dados de presença-ausência. nmed1.np. $2<-(($ dat1 $)-(n p)) \wedge 2$

nmed2.np. $2<-((\operatorname{dat} 2)-(\mathrm{np}))^{\wedge} 2$ 
nmed3.np. $2<-(($ dat3 $)-(n p)){ }^{\wedge} 2$

nmed4.np. $2<-((\operatorname{dat} 4)-(\mathrm{np}))^{\wedge} 2$

nmed5.np. $2<-((\operatorname{dat5})-(\mathrm{np}))^{\wedge} 2$

nmed6.np. $2<-((\operatorname{dat} 6)-(\mathrm{np}))^{\wedge} 2$

ajmedbv6<-(nmed1.np. 2+nmed2.np.2+nmed3.np. 2+nmed4.np. 2+nmed5.np. 2+nmed6.np. 2)/np

ajmedbv6

\# Valor de qui-quadrado tabelado, com 5 (cinco) graus de liberdade

e com nível de significância igual a 0,05.

qui. 2. tab. $5<-11.070$

qui.2.tab.5

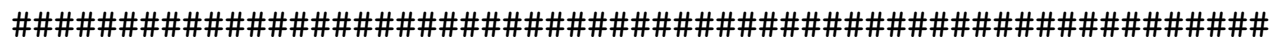

Observações do talhão 6 da Fazenda Bela Vista, período de frutificação.

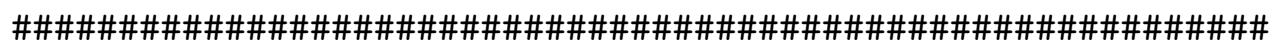

\#\# Ramos \#\#

Data de amostragem (19/09/2003).

bv6frutramos $<-c(1,0,0,0,0,1,0,0,1,0,0,0,0,0)$

med.r<-mean (bv6frutramos)

med.r

var. $r<-\operatorname{var}($ bv6frutramos)

var.r

\# Média geral e média das variâncias.

p. $r<-$ med. $r$

p.r

medvar.r<-var.r

medvar.r 
\# Média e variância para a distribuição binomial.

np.r<- $14 *$ p.r

$\mathrm{np} . \mathrm{r}$

npq. r<- $14 *$ p.r*(1-p.r)

$\mathrm{npq} \cdot \mathrm{r}$

\# Aplicação do teste de qui-quadrado para verificar a eficiência do ajuste da distribuição binomial aos dados de presença-ausência. nmed.np. $2 . r<-((14 * \text { med.r })-(n p . r))^{\wedge} 2$

ajmedbv6.r<-(nmed.np.2.r)/np.r

ajmedbv6.r

\#\# Frutos \#\#

Data de amostragem (19/09/2003).

bv6frutfrutos $<-c(0,0,0,0,0,0,0,0,0,0,0,0,0,0)$

med.f $<-$ mean (bv6frutfrutos)

med.f

$\operatorname{var} . \mathrm{f}<-\operatorname{var}($ bv6frutfrutos)

$\operatorname{var} . f$

\# Média geral e média das variâncias.

p. $f<-\operatorname{med} . f$

p.f

medvar.f<-var.f

medvar.f

\# Média e variância para a distribuição binomial.

$\mathrm{np} . \mathrm{f}<-14 * \mathrm{p} . \mathrm{f}$

$\mathrm{np} . \mathrm{f}$

npq.f<- $14 *$ p.f*(1-p.f)

npq. $f$ 
\# Aplicação do teste de qui-quadrado para verificar a eficiência do ajuste da distribuição binomial aos dados de presença-ausência. nmed.np. $2 . f<-((14 * \text { med.f })-(n p . f))^{\wedge} 2$

ajmedbv6.f<-(nmed.np.2.f)/np.f

ajmedbv6.f

\# Valor de qui-quadrado tabelado, com 1 (um) grau de liberdade e com nível de significância igual a 0,05.

qui.2.tab. $1<-3.841$

qui.2.tab.1

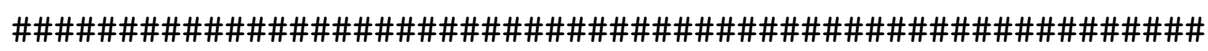

Observações do talhão 7 da Fazenda Bela Vista, período vegetativo.

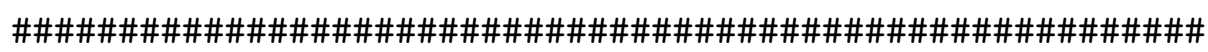

\# Primeira data de amostragem (21/05/2003).

bv7veg $1<-c(0,0,0,0,0,0,0,0,0,0,0,0,0,0)$

med1<-mean (bv7veg1)

med1

$\operatorname{var} 1<-\operatorname{var}($ bv7veg1)

$\operatorname{var} 1$

\# Segunda data de amostragem (11/06/2003).

bv7veg $2<-c(0,0,0,0,0,0,0,0,0,0,0,0,0,0)$

med2<-mean (bv7veg2)

med2

$\operatorname{var} 2<-\operatorname{var}($ bv7veg2)

$\operatorname{var} 2$

\# Terceira data de amostragem (21/06/2003). 


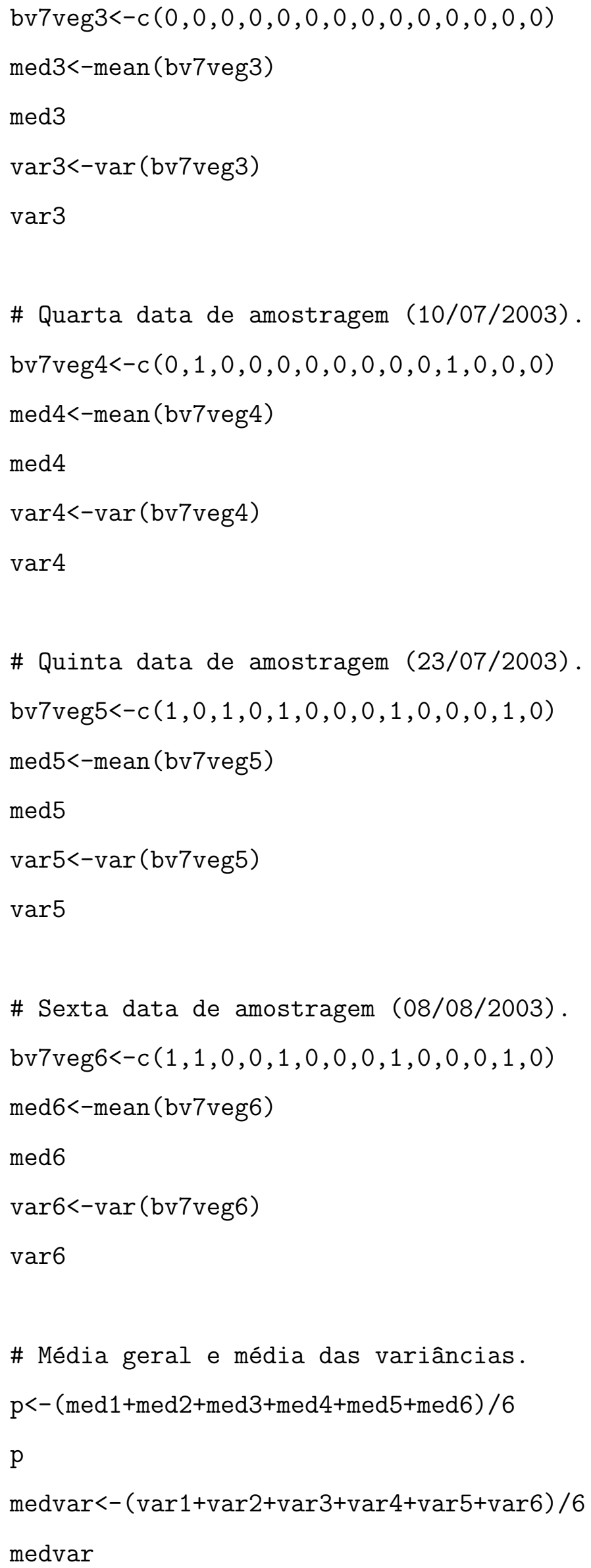


\# Média e variância para a distribuição binomial.

$\mathrm{np}<-14 * \mathrm{p}$

$\mathrm{np}$

$\mathrm{npq}<-14 * \mathrm{p} *(1-\mathrm{p})$

$\mathrm{npq}$

\# Aplicação do teste de qui-quadrado para verificar a eficiência do ajuste da distribuição binomial aos dados de presença-ausência. nmed1.np. $2<-((14 * \operatorname{med} 1)-(\mathrm{np}))^{\wedge} 2$ nmed2.np. $2<-((14 * \operatorname{med} 2)-(\mathrm{np}))^{\wedge} 2$ nmed3.np. $2<-((14 * \text { med3 })-(\mathrm{np}))^{\wedge} 2$ $\mathrm{nmed} 4 . \mathrm{np} .2<-((14 * \operatorname{med} 4)-(\mathrm{np})))^{\wedge}$ nmed5.np. $2<-((14 * \text { med5 })-(\mathrm{np}))^{\wedge} 2$ nmed6.np. $2<-((14 * \operatorname{med} 6)-(\mathrm{np})))^{\wedge}$ ajmedbv7<-(nmed1.np.2+nmed2.np.2+nmed3.np.2+nmed4.np. 2+nmed5.np. 2+nmed6.np. 2)/np ajmedbv7

\# Valor de qui-quadrado tabelado, com 5 (cinco) graus de liberdade e com nível de significância igual a 0,05. qui. 2. tab. $5<-11.070$ qui.2.tab. 5

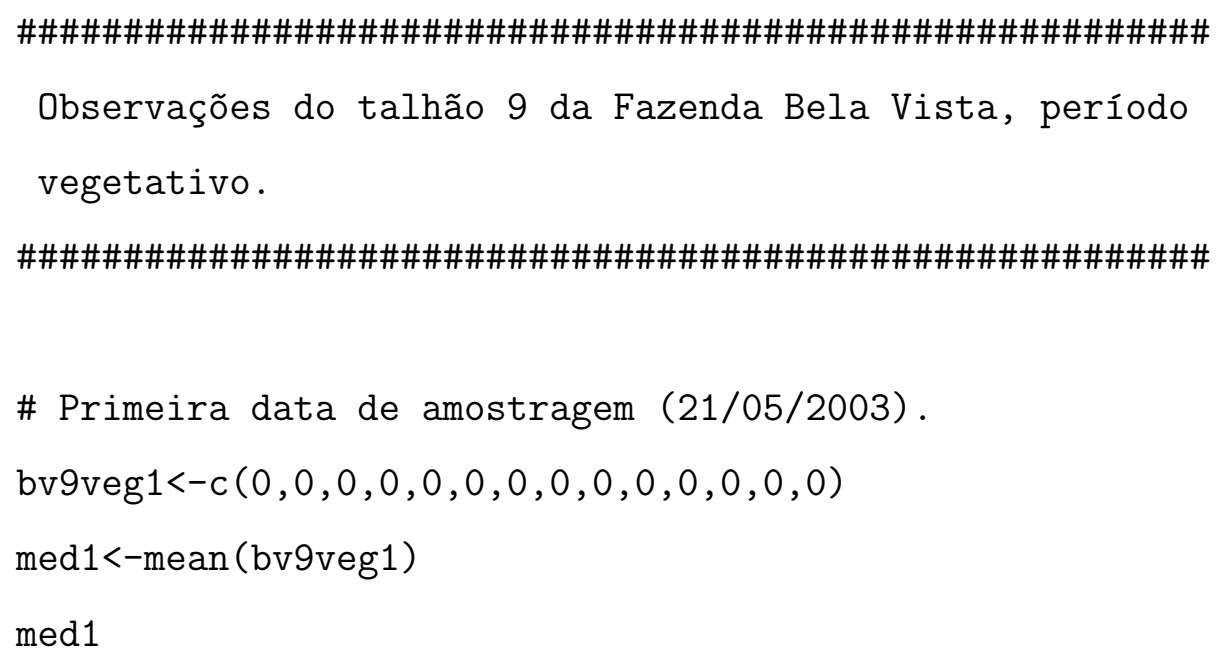


$\operatorname{var} 1<-\operatorname{var}($ bv9veg1)

$\operatorname{var} 1$

\# Segunda data de amostragem (11/06/2003).

bv9veg $2<-c(1,0,1,0,0,0,0,0,0,1,0,0,0,0)$

med2<-mean (bv9veg2)

med2

var2<-var (bv9veg2)

$\operatorname{var} 2$

\# Terceira data de amostragem (21/06/2003).

bv9veg $3<-c(0,0,0,0,0,0,0,0,1,0,0,0,0,0)$

med3<-mean (bv9veg3)

med3

var3<-var (bv9veg3)

var3

\# Quarta data de amostragem (10/07/2003).

bv9veg $4<-c(1,1,1,1,1,1,0,0,1,1,1,1,1,1)$

med4<-mean (bv9veg4)

$\operatorname{med} 4$

$\operatorname{var} 4<-\operatorname{var}($ bv9veg4)

$\operatorname{var} 4$

\# Quinta data de amostragem (23/07/2003).

bv9veg5<-c $(1,0,1,0,1,1,1,1,0,1,1,1,1,1)$

med5<-mean (bv9veg5)

med5

$\operatorname{var} 5<-\operatorname{var}($ bv9veg5)

$\operatorname{var5}$

\# Sexta data de amostragem (08/08/2003). 


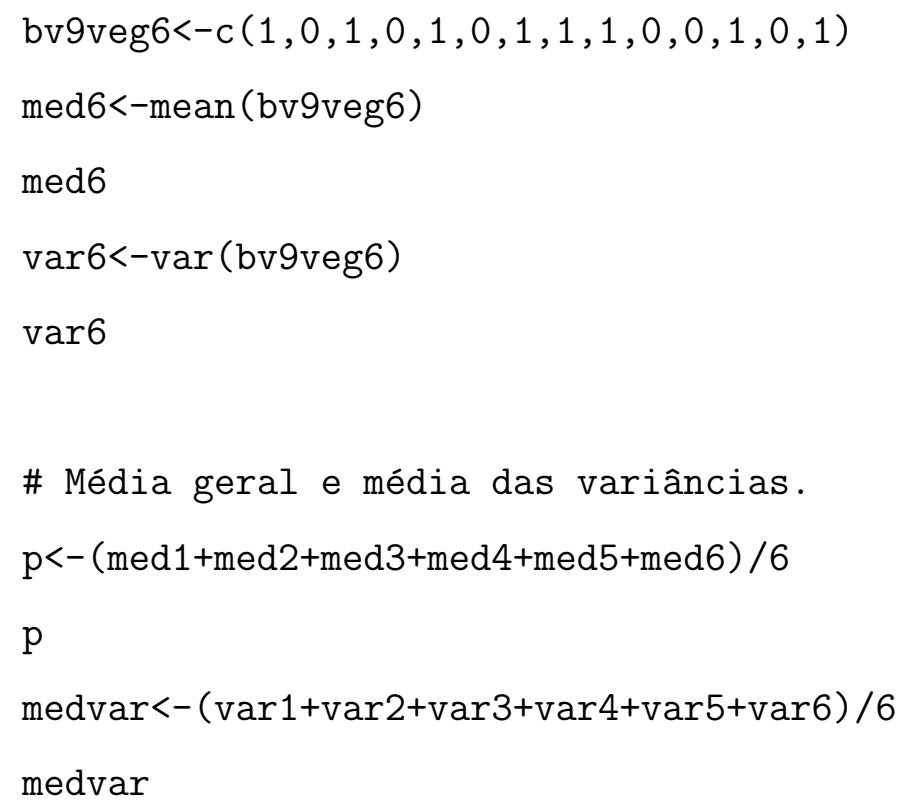


qui.2.tab.5

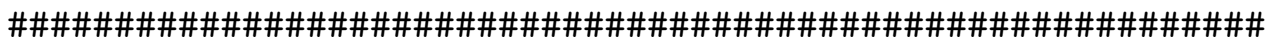
Observações do talhão 9 da Fazenda Bela Vista, período de frutificação.

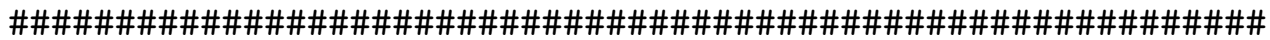

\#\# Ramos \#\#

\# Primeira data de amostragem (27/08/2003).

bv9frutram $1<-c(0,0,0,0,0,0,0,0,0,0,0,0,0,0)$

med1. $r<-$ mean (bv9frutram1)

$\operatorname{med} 1 . r$

$\operatorname{var} 1 . r<-\operatorname{var}($ bv9frutram1)

$\operatorname{var} 1 . r$

\# Segunda data de amostragem (18/09/2003).

bv9frutram $2<-c(0,0,0,0,0,0,0,0,0,0,0,0,0,0)$

med2. $r<-$ mean (bv9frutram2)

$\operatorname{med} 2 . r$

$\operatorname{var} 2 . r<-\operatorname{var}($ bv9frutram2)

$\operatorname{var} 2 \cdot r$

\# Terceira data de amostragem (04/10/2003).

bv9frutram $3<-c(0,0,0,0,0,0,0,0,0,0,0,0,0,0)$

med3. $r<-$ mean (bv9frutram3)

$\operatorname{med} 3 . r$

var3.r<-var (bv9frutram3)

var3.r

\# Média geral e média das variâncias.

p. $r<-(\operatorname{med} 1 . r+\operatorname{med} 2 . r+\operatorname{med} 3 \cdot r) / 3$ 
p.r

medvar. $r<-(\operatorname{var} 1 . r+\operatorname{var} 2 \cdot r+\operatorname{var} 3 \cdot r) / 3$

medvar.r

\# Média e variância para a distribuição binomial.

$\mathrm{np} . \mathrm{r}<-14 * \mathrm{p} . \mathrm{r}$

$\mathrm{np} . \mathrm{r}$

npq. r<- $14 *$ p.r*(1-p.r)

npq.r

\# Aplicação do teste de qui-quadrado para verificar a eficiência do ajuste da distribuição binomial aos dados de presença-ausência. nmed1.np.2.r<-((14*med1.r)-(np.r) $)^{\wedge} 2$ nmed2.np.2.r<-((14*med2.r) $-(\mathrm{np} . \mathrm{r}))^{\wedge} 2$ nmed3.np. 2.r<-((14*med3.r)-(np.r))^2

ajmedbv9.r<-(nmed1.np.2.r+nmed2.np.2.r+nmed3.np.2.r)/np.r ajmedbv9.r

\#\# Frutos \#\#

\# Primeira data de amostragem (27/08/2003).

bv9frutfrut $1<-c(0,0,1,0,0,0,0,0,0,0,0,0,1,0)$

med1.f<-mean (bv9frutfrut1)

$\operatorname{med} 1 . \mathrm{f}$

$\operatorname{var} 1 . \mathrm{f}<-\operatorname{var}($ bv9frutfrut1)

$\operatorname{var} 1 . f$

\# Segunda data de amostragem (18/09/2003).

bv9frutfrut $2<-c(0,0,0,1,0,0,0,0,0,0,0,0,1,0)$

med2.f<-mean (bv9frutfrut2)

$\operatorname{med} 2 . \mathrm{f}$

$\operatorname{var} 2 . \mathrm{f}<-\operatorname{var}$ (bv9frutfrut2) 
$\operatorname{var} 2 . \mathrm{f}$

\# Terceira data de amostragem (04/10/2003).

bv9frutfrut $3<-c(0,0,0,0,0,0,0,0,0,0,0,0,0,0)$

med3.f<-mean (bv9frutfrut3)

$\operatorname{med} 3 . f$

var3.f<-var(bv9frutfrut3)

$\operatorname{var} 3 . \mathrm{f}$

\# Média geral e média das variâncias.

p. $f<-(\operatorname{med} 1 . f+\operatorname{med} 2 . f+\operatorname{med} 3 . f) / 3$

p.f

medvar.f<-( $\operatorname{var} 1 . f+\operatorname{var} 2 . f+\operatorname{var} 3 . f) / 3$

medvar.f

\# Média e variância para a distribuição binomial.

np.f<- $14 *$ p.f

$\mathrm{np} . \mathrm{f}$

npq.f<- $14 *$ p.f*(1-p.f)

npq. $f$

\# Aplicação do teste de qui-quadrado para verificar a eficiência do ajuste da distribuição binomial aos dados de presença-ausência. nmed1.np. $2 . f<-((14 * \operatorname{med} 1 . f)-(n p . f))^{\wedge} 2$

nmed2.np. $2 . f<-((14 * \operatorname{med} 2 . f)-(n p . f))^{\wedge} 2$

nmed3.np. $2 . f<-((14 * \operatorname{med} 3 . f)-(n p . f))^{\wedge} 2$

ajmedbv9.f<-(nmed1.np.2.f+nmed2.np.2.f+nmed3.np.2.f)/np.f

ajmedbv9.f

\# Valor de qui-quadrado tabelado, com 2 (dois) graus de liberdade e com nível de significância igual a 0,05.

qui. 2. tab. $2<-5.991$ 
qui.2.tab.2

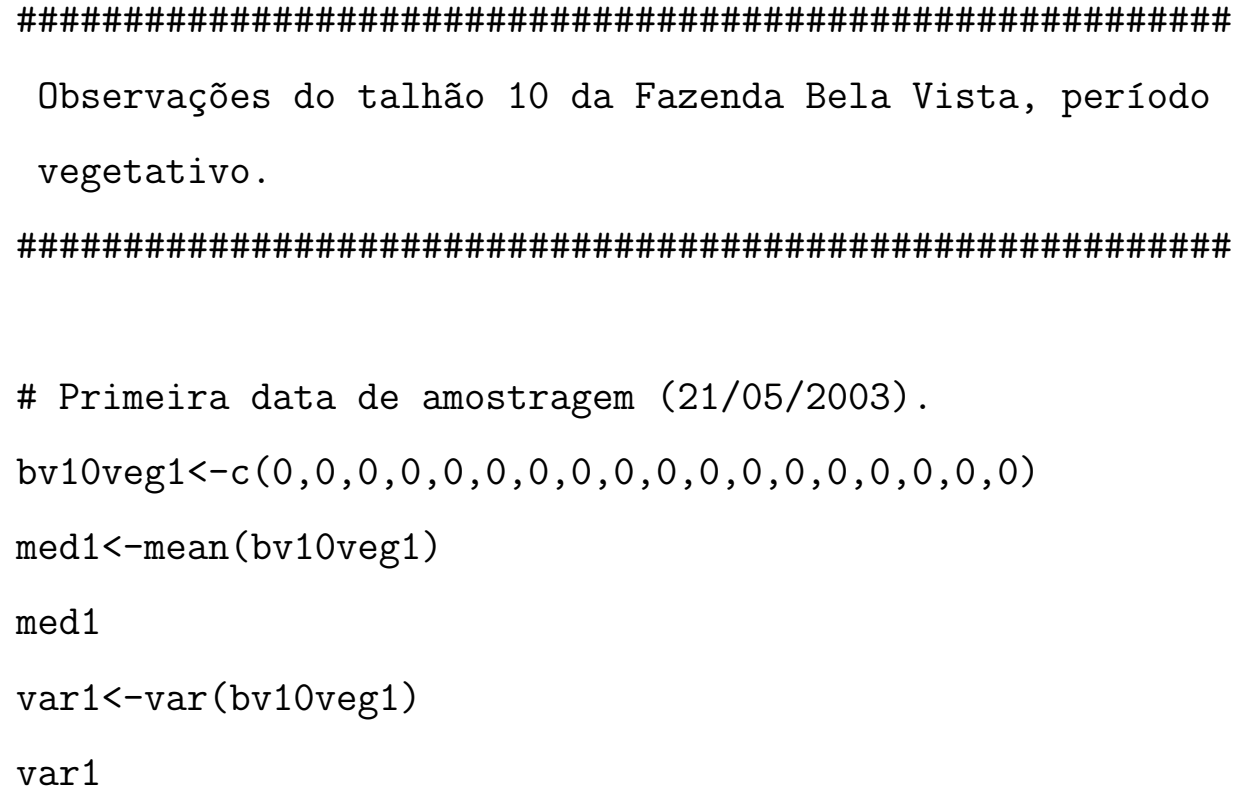




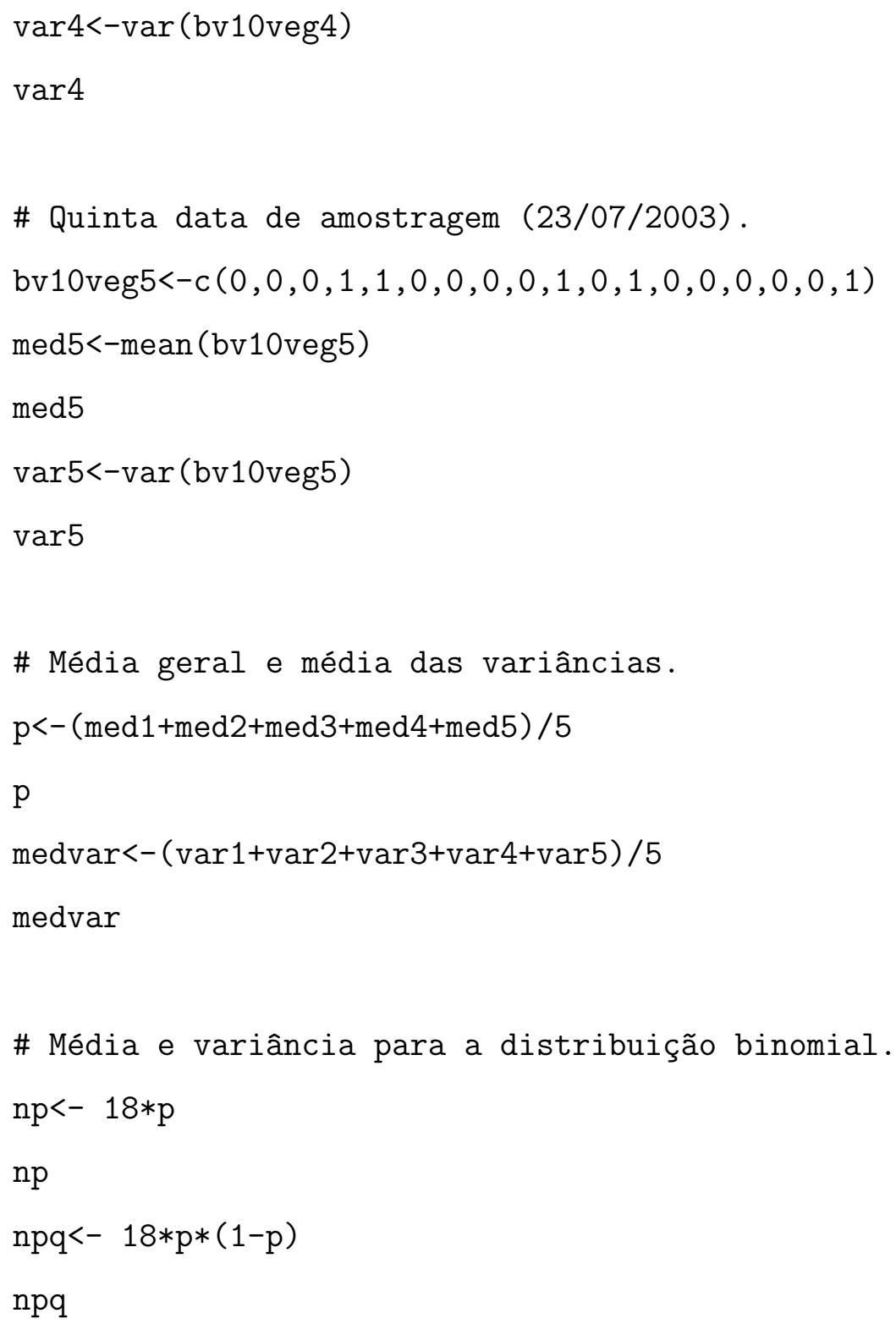


qui.2.tab. $4<-9.488$

qui.2.tab. 4

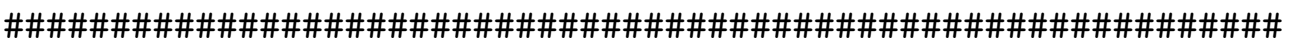

Observações do talhão 10 da Fazenda Bela Vista, período de frutificação .

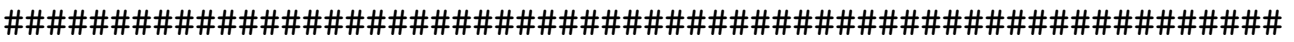

\#\# Ramos \#\#

\# Primeira data de amostragem (08/08/2003).

bv10frutram $1<-c(1,0,0,1,0,1,0,1,1,0,1,0,1,0,0,0,0,0)$

med1.r<-mean(bv10frutram1)

$\operatorname{med} 1 . r$

$\operatorname{var} 1 . r<-\operatorname{var}($ bv10frutram1)

$\operatorname{var} 1 . r$

\# Segunda data de amostragem (28/08/2003).

bv10frutram $2<-c(0,0,0,0,0,0,0,0,0,0,0,0,0,0,0,0,0,0)$

med2.r<-mean(bv10frutram2)

$\operatorname{med} 2 \cdot r$

$\operatorname{var} 2 . r<-\operatorname{var}($ bv10frutram2)

$\operatorname{var} 2 . r$

\# Terceira data de amostragem (19/09/2003).

bv10frutram $3<-c(0,0,0,0,0,0,0,0,0,0,0,0,0,0,0,0,0,0)$

med3.r $<-$ mean (bv10frutram3)

$\operatorname{med3.r}$

var3. $r<-\operatorname{var}($ bv10frutram3)

$\operatorname{var} 3 . r$

\# Quarta data de amostragem (10/10/2003). 
bv10frutram $4<-c(0,0,0,0,0,0,0,0,0,0,0,0,0,0,0,0,0,0)$

med4. $r<-$ mean (bv10frutram4)

$\operatorname{med} 4 . r$

$\operatorname{var} 4 . r<-\operatorname{var}($ bv10frutram4)

$\operatorname{var} 4 . r$

\# Média geral e média das variâncias.

p. $r<-(\operatorname{med} 1 . r+\operatorname{med} 2 . r+\operatorname{med} 3 . r+\operatorname{med} 4 . r) / 4$

p.r

medvar $. r<-(\operatorname{var} 1 . r+\operatorname{var} 2 \cdot r+\operatorname{var} 3 \cdot r+\operatorname{var} 4 \cdot r) / 4$

medvar.r

\# Média e variância para a distribuição binomial.

np.r $<-18 *$ p.r

np.r

npq. $r<-18 *$ p.r*(1-p.r)

$\mathrm{npq} \cdot \mathrm{r}$

\# Aplicação do teste de qui-quadrado para verificar a eficiência do ajuste da distribuição binomial aos dados de presença-ausência.

nmed1.np.2.r<-((18*med1.r)-(np.r) )^2

nmed2.np.2.r<-((18*med2.r)-(np.r) $)^{\wedge} 2$

nmed3.np. 2.r<-((18*med3.r)-(np.r) $)^{\wedge} 2$

nmed4.np.2.r<-((18*med4.r)-(np.r))^2

ajmedbv10.r<-(nmed1.np.2.r+nmed2.np.2.r+nmed3.np.2.r+nmed4.np.2.r)/np.r ajmedbv10.r

\#\# Frutos \#\#

\# Primeira data de amostragem (08/08/2003).

bv10frutfrut $1<-c(0,0,0,0,0,0,0,0,0,0,0,0,0,0,0,0,0,0)$

med1.f<-mean (bv10frutfrut1) 
$\operatorname{med} 1 . f$

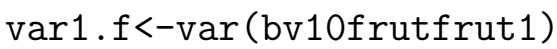

$\operatorname{var} 1 . f$

\# Segunda data de amostragem (28/08/2003).

bv10frutfrut $2<-c(1,0,1,0,0,1,0,0,0,0,0,0,0,0,1,0,0,0)$

med2.f<-mean(bv10frutfrut2)

$\operatorname{med} 2 . f$

$\operatorname{var} 2 . f<-\operatorname{var}($ bv10frutfrut2)

$\operatorname{var} 2 . f$

\# Terceira data de amostragem (19/09/2003).

bv10frutfrut $3<-c(0,0,0,1,0,0,0,0,0,0,1,0,0,0,0,0,0,1)$

med3.f<-mean (bv10frutfrut3)

$\operatorname{med3.f}$

var3.f<-var(bv10frutfrut3)

$\operatorname{var} 3 . f$

\# Quarta data de amostragem (10/10/2003).

bv10frutfrut $4<-c(0,0,0,1,0,0,0,0,0,0,1,0,0,0,0,0,0,0)$

med4.f<-mean(bv10frutfrut4)

$\operatorname{med} 4 . \mathrm{f}$

$\operatorname{var} 4 . f<-\operatorname{var}($ bv10frutfrut4)

$\operatorname{var} 4 . f$

\# Média geral e média das variâncias.

p.f $<-(\operatorname{med} 1 . f+\operatorname{med} 2 . f+\operatorname{med} 3 . f+\operatorname{med} 4 . f) / 4$

p.f

medvar.f<- $(\operatorname{var} 1 . f+\operatorname{var} 2 . f+\operatorname{var} 3 . f+\operatorname{med} 4 . f) / 4$

medvar.f

\# Média e variância para a distribuição binomial. 
$\mathrm{np} . \mathrm{f}<-18 * \mathrm{p} . \mathrm{f}$

np. f

npq.f<- $18 *$ p.f*(1-p.f)

npq.f

\# Aplicação do teste de qui-quadrado para verificar a eficiência do ajuste da distribuição binomial aos dados de presença-ausência. nmed1.np. $2 . f<-((18 * \operatorname{med} 1 . f)-(n p . f)) ` 2$

nmed2.np. $2 . f<-((18 * \operatorname{med} 2 . f)-(n p . f))^{\wedge} 2$

nmed3.np.2.f<-((18*med3.f $)-($ np.f $))^{\wedge} 2$

nmed4.np. $2 . f<-((18 * \operatorname{med} 4 . f)-(n p . f))^{\wedge} 2$

ajmedbv10.f<-(nmed1.np.2.f+nmed2.np.2.f+nmed3.np.2.f+nmed4.np.2.f $) / n p . f$ ajmedbv10.f

\# Valor de qui-quadrado tabelado, com 3 (três) graus de liberdade e com nível de significância igual a 0,05.

qui. 2. tab. $3<-7.815$

qui.2.tab.3

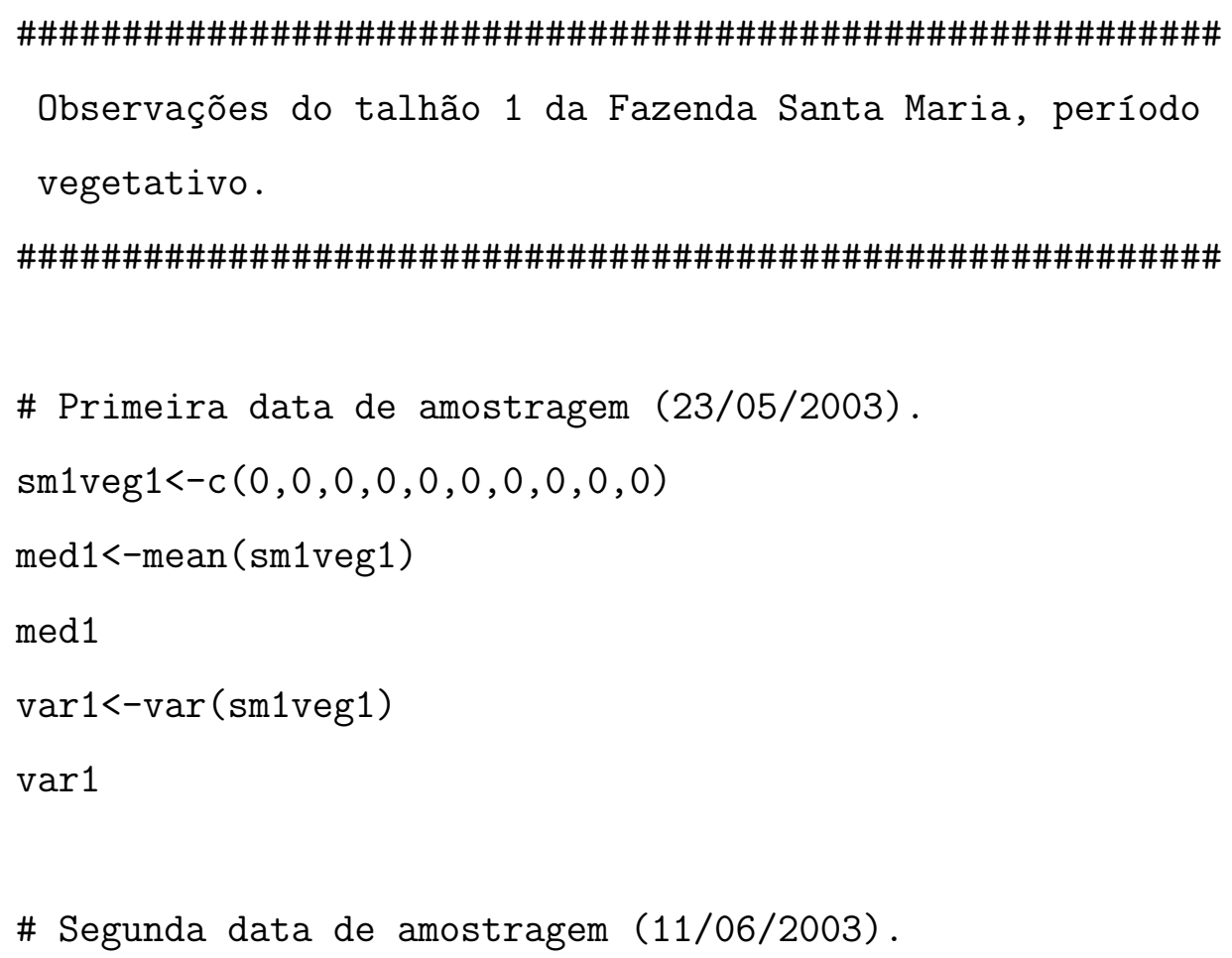




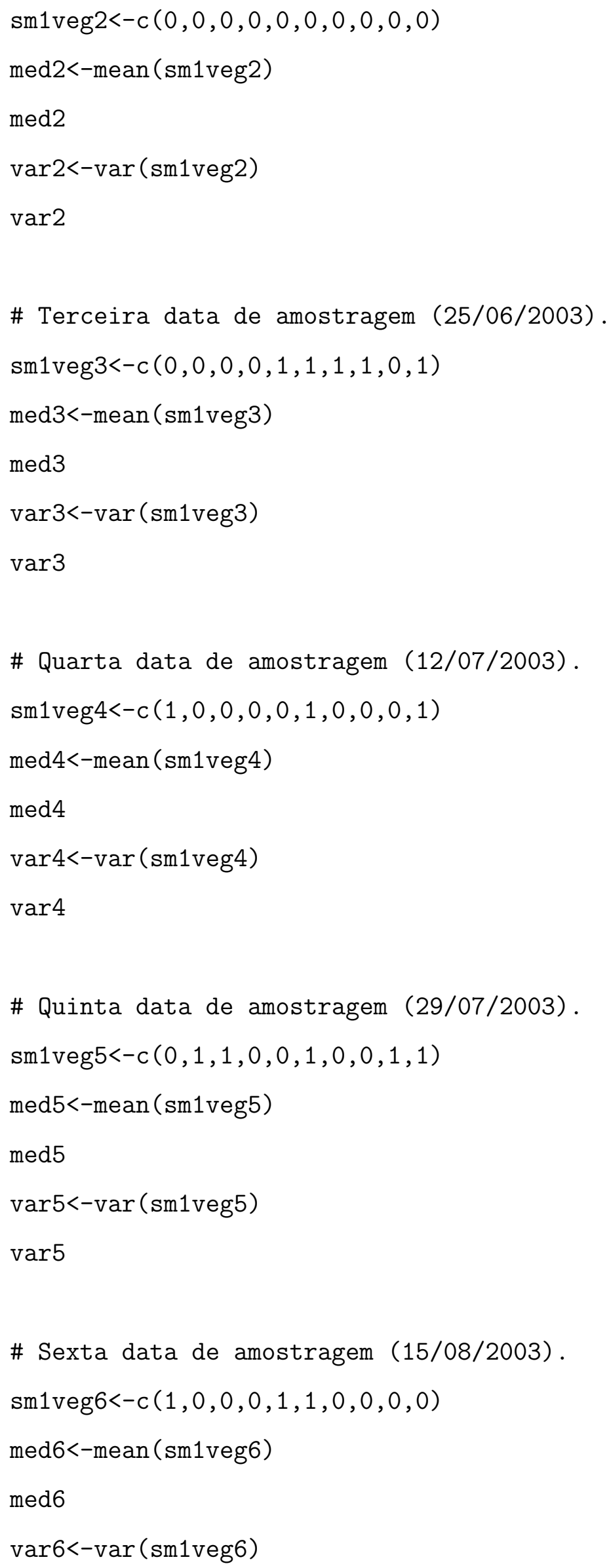


$\operatorname{var} 6$

\# Média geral e média das variâncias.

$\mathrm{p}<-(\operatorname{med} 1+\operatorname{med} 2+\operatorname{med} 3+\operatorname{med} 4+\operatorname{med} 5+\operatorname{med} 6) / 6$

$\mathrm{p}$

medvar<- (var1+var2+var3+var4+var5+var6) /6

medvar

\# Média e variância para a distribuição binomial.

$\mathrm{np}<-10 * \mathrm{p}$

$\mathrm{np}$

$\mathrm{npq}<-10 * \mathrm{p} *(1-\mathrm{p})$

$\mathrm{npq}$

\# Aplicação do teste de qui-quadrado para verificar a eficiência do ajuste da distribuição binomial aos dados de presença-ausência. nmed1.np. $2<-((10 * \operatorname{med} 1)-(\mathrm{np})))^{\wedge}$

nmed2.np. $2<-((10 * \operatorname{med} 2)-(\mathrm{np})){ }^{\wedge} 2$

nmed3.np. $2<-((10 * \operatorname{med} 3)-(\mathrm{np}))^{\wedge} 2$

nmed4.np. $2<-((10 * \operatorname{med} 4)-(\mathrm{np})))^{\wedge}$

nmed5.np. $2<-((10 * \text { med5 })-(\mathrm{np}))^{\wedge} 2$

nmed6.np. $2<-((10 * \text { med6 })-(\mathrm{np}))^{\wedge} 2$

ajmedsm1<-(nmed1.np.2+nmed2.np.2+nmed3.np.2+nmed4.np.2+nmed5.np.2+nmed6.np.2)/np ajmedsm1

\# Valor de qui-quadrado tabelado, com 5 (cinco) graus de liberdade e com nível de significância igual a 0,05.

qui. 2. tab. $5<-11.070$

qui.2.tab.5 


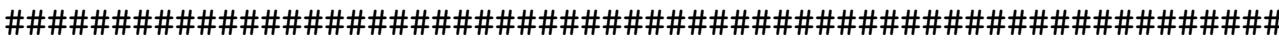 \\ Observações do talhão 1 da Fazenda Santa Maria, período de frutificação.}

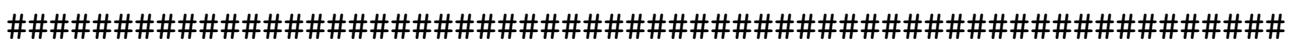

\#\# Ramos \#\#

Data de amostragem (19/09/2003).

sm1frutram $<-c(0,0,0,0,0,0,0,0,0,0,0,0,0,0)$

med.r<-mean (sm1frutram)

med.r

$\operatorname{var} . r<-\operatorname{var}($ sm1frutram)

$\operatorname{var} . r$

\# Média geral e média das variâncias.

p.r<-med.r

p.r

medvar.r<-var.r

medvar.r

\# Média e variância para a distribuição binomial.

np.r<- $10 *$ p.r

$\mathrm{np} . \mathrm{r}$

npq. $r<-10 * p \cdot r *(1-p \cdot r)$

npq.r

\# Aplicação do teste de qui-quadrado para verificar a eficiência do ajuste da distribuição binomial aos dados de presença-ausência. nmed.np. $2 . r<-((10 *$ med.r $)-(n p . r)) ` 2$

ajmedsm1.r<-(nmed.np.2.r)/np.r ajmedsm1.r 
\#\# Frutos \#\#

Data de amostragem (19/09/2003).

sm1frutfrut $<-c(0,0,0,0,0,0,0,0,0,0,0,0,0,0)$

med.f<-mean (sm1frutfrut)

med.f

$\operatorname{var} . \mathrm{f}<-\operatorname{var}($ sm1frutfrut)

$\operatorname{var} . \mathrm{f}$

\# Média geral e média das variÂncias.

p.f $<-$ med.f

p.f

medvar.f<-var.f

medvar.f

\# Média e variância para a distribuição binomial.

$\mathrm{np} . \mathrm{f}<-10 * \mathrm{p} . \mathrm{f}$

$\mathrm{np} . \mathrm{f}$

npq.f<- $10 *$ p.f*(1-p.f)

npq. $f$

\# Aplicação do teste de qui-quadrado para verificar a eficiência do ajuste da distribuição binomial aos dados de presença-ausência. nmed.np. $2 . f<-((10 * \text { med.f })-(n p . f))^{\wedge} 2$

ajmedsm1.f<-(nmed.np.2.f)/np.f

ajmedsm1.f

\# Valor de qui-quadrado tabelado, com 1 (um) grau de liberdade e com nível de significância igual a 0,05.

qui. 2. tab. $1<-3.841$

qui.2.tab.1 


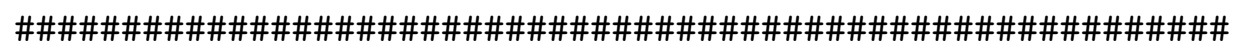

Observações do talhão 2 da Fazenda Santa Maria, período vegetativo.

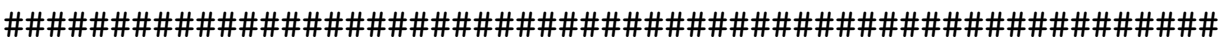

\# Primeira data de amostragem (23/05/2003).

sm2veg1<-c $(0,0,0,0,0,0,0,0,0,0)$

med1<-mean ( sm2veg1)

med1

$\operatorname{var} 1<-\operatorname{var}(\operatorname{sm} 2 \operatorname{veg} 1)$

$\operatorname{var} 1$

\# Segunda data de amostragem (11/06/2003).

sm2veg2<-c $(0,0,0,0,0,0,0,0,0,0)$

med2<-mean (sm2veg2)

med2

$\operatorname{var} 2<-\operatorname{var}(\mathrm{sm} 2 \mathrm{veg} 2)$

$\operatorname{var} 2$

\# Terceira data de amostragem (25/06/2003).

sm2veg $3<-c(1,0,1,0,0,0,1,0,1,0)$

med3<-mean (sm2veg3)

med3

$\operatorname{var} 3<-\operatorname{var}($ sm2veg3)

$\operatorname{var} 3$

\# Quarta data de amostragem (09/07/2003).

sm2veg $4<-c(0,1,0,0,1,0,0,0,0,1)$

med4<-mean (sm2veg4)

med4

$\operatorname{var} 4<-\operatorname{var}(\operatorname{sm} 2 \operatorname{veg} 4)$

$\operatorname{var} 4$ 
\# Quinta data de amostragem (22/07/2003).

sm2veg $5<-c(0,0,1,0,0,0,0,1,0,0)$

med5<-mean ( sm2veg5)

med5

$\operatorname{var} 5<-\operatorname{var}($ sm2veg5)

$\operatorname{var} 5$

\# Média geral e média das variâncias.

$\mathrm{p}<-(\operatorname{med} 1+\operatorname{med} 2+\operatorname{med} 3+\operatorname{med} 4+\operatorname{med} 5) / 5$

$\mathrm{p}$

medvar<- $(\operatorname{var} 1+\operatorname{var} 2+\operatorname{var} 3+\operatorname{var} 4+\operatorname{var} 5) / 5$

medvar

\# Média e variância para a distribuição binomial.

$\mathrm{np}<-10 * \mathrm{p}$

$\mathrm{np}$

$\mathrm{npq}<-10 * \mathrm{p} *(1-\mathrm{p})$

npq

\# Aplicação do teste de qui-quadrado para verificar a eficiência do ajuste da distribuição binomial aos dados de presença-ausência.

nmed1.np. $2<-((10 * \operatorname{med} 1)-(\mathrm{np})))^{\wedge}$

nmed2.np. $2<-((10 * \operatorname{med} 2)-(\mathrm{np})))^{\wedge}$

nmed3.np. $2<-((10 * \operatorname{med} 3)-(\mathrm{np})))^{\wedge}$

nmed4.np. $2<-((10 * \operatorname{med} 4)-(\mathrm{np})))^{\wedge}$

nmed5.np. $2<-((10 * \text { med5 })-(\mathrm{np}))^{\wedge} 2$

ajmedsm2<-(nmed1.np.2+nmed2.np.2+nmed3.np.2+nmed4.np.2+nmed5.np.2)/np

ajmedsm2

\# Valor de qui-quadrado tabelado, com 4 (quatro) graus de liberdade e com nível de significância igual a 0,05. 
qui.2.tab. $4<-9.488$

qui.2.tab. 4

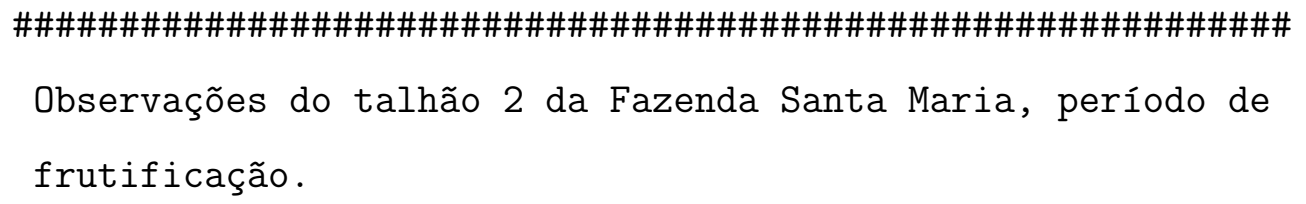


np.r

npq. $r<-10 *$ p.r*(1-p.r)

npq.r

\# Aplicação do teste de qui-quadrado para verificar a eficiência do ajuste da distribuição binomial aos dados de presença-ausência. nmed1.np.2.r<-((10*med1.r $)-(n p . r)) ` 2$

nmed2.np.2.r<-((10*med2.r $)-(n p \cdot r))^{\wedge} 2$

ajmedsm2.r<-(nmed1.np.2.r+nmed2.np.2.r)/np.r

ajmedsm2.r

\#\# Frutos \#\#

\# Primeira data de amostragem (09/08/2003).

sm2frutfrut $1<-c(0,0,0,0,0,0,0,0,0,0,0,0,0,0)$

med1.f<-mean (sm2frutfrut1)

$\operatorname{med} 1 . \mathrm{f}$

$\operatorname{var} 1 . f<-\operatorname{var}($ sm2frutfrut1)

$\operatorname{var} 1 . \mathrm{f}$

\# Segunda data de amostragem (19/09/2003).

sm2frutfrut $2<-c(0,0,0,0,0,0,0,0,0,0,0,0,0,0)$

med2.f<-mean(sm2frutfrut2)

$\operatorname{med} 2 . \mathrm{f}$

$\operatorname{var} 2 . \mathrm{f}<-\operatorname{var}($ sm2frutfrut2)

$\operatorname{var} 2 . f$

\# Média geral e média das variâncias.

p.f $<-(\operatorname{med} 1 . f+\operatorname{med} 2 . f) / 2$

p.f

medvar.f<- $(\operatorname{var} 1 . f+\operatorname{var} 2 . f) / 2$

medvar.f 
\# Média e variância para a distribuição binomial.

np. $f<-10 *$ p. $f$

$\mathrm{np} . \mathrm{f}$

npq.f<- $10 *$ p.f*(1-p.f)

npq. $f$

\# Aplicação do teste de qui-quadrado para verificar a eficiência do ajuste da distribuição binomial aos dados de presença-ausência. nmed1.np. $2 . f<-((10 * \operatorname{med} 1 . f)-(n p . f))^{\wedge} 2$

nmed2.np.2.f<-((10*med2.f $)-($ np.f $))^{\wedge} 2$

ajmedsm2.f<-(nmed1.np.2.f+nmed2.np.2.f)/np.f

ajmedsm2.f

\# Valor de qui-quadrado tabelado, com 1 (um) grau de \# liberdade e com nível de significância igual a 0,05.

qui. 2. tab. $1<-3.841$

qui.2.tab.1

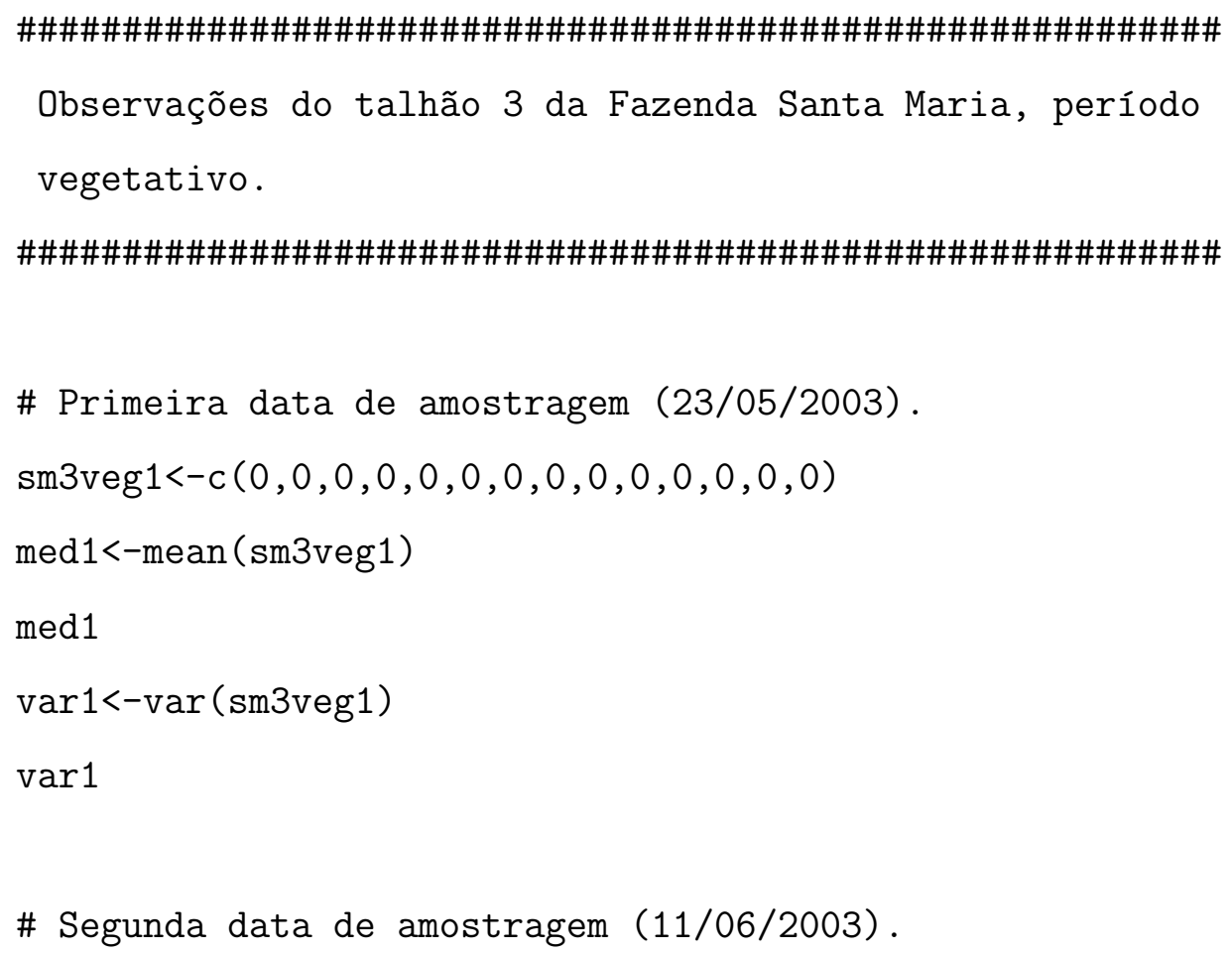




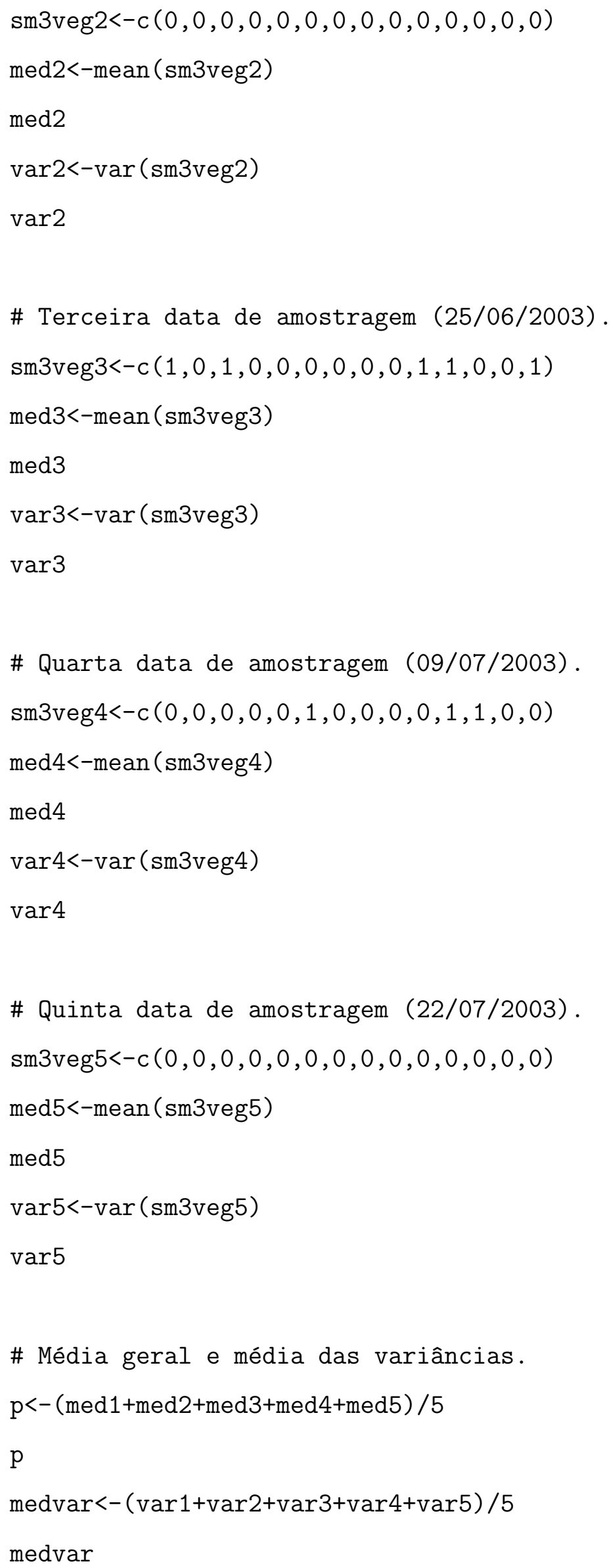


\# Média e variância para a distribuição binomial.

$\mathrm{np}<-14 * \mathrm{p}$

$\mathrm{np}$

$\mathrm{npq}<-14 * \mathrm{p} *(1-\mathrm{p})$

$\mathrm{npq}$

\# Aplicação do teste de qui-quadrado para verificar a eficiência do ajuste da distribuição binomial aos dados de presença-ausência. nmed1.np. $2<-((14 * \operatorname{med} 1)-(\mathrm{np})))^{\wedge}$ nmed2 $\cdot \mathrm{np} \cdot 2<-((14 * \operatorname{med} 2)-(\mathrm{np})))^{\wedge}$ nmed3.np. $2<-((14 * \operatorname{med} 3)-(\mathrm{np})))^{\wedge}$ nmed4.np. $2<-((14 * \operatorname{med} 4)-(\mathrm{np})))^{\wedge}$ nmed5.np. $2<-((14 * \text { med5 })-(\mathrm{np}))^{\wedge} 2$ ajmedsm3<-(nmed1.np.2+nmed2.np.2+nmed3.np.2+nmed4.np.2+nmed5.np. 2)/np ajmedsm3

\# Valor de qui-quadrado tabelado, com 4 (quatro) graus de liberdade e com nível de significância igual a 0,05.

qui. 2. tab. $4<-9.488$

qui.2.tab. 4

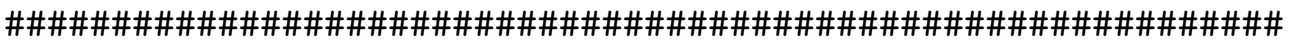

Observações do talhão 3 da Fazenda Santa Maria, período de frutificação.

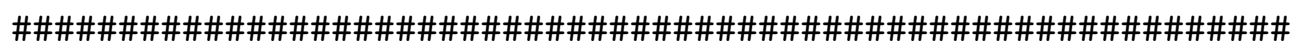

\#\# Ramos \#\#

\# Primeira data de amostragem (07/08/2003).

sm3frutram $1<-c(0,0,0,0,0,0,0,0,0,0,0,0,0,0)$

med1. $r<-$ mean (sm3frutram1) 
$\operatorname{med} 1 . r$

$\operatorname{var} 1 . r<-\operatorname{var}(\mathrm{sm} 3 \mathrm{frutram} 1)$

$\operatorname{var} 1 . r$

\# Segunda data de amostragem (19/09/2003).

sm3frutram $2<-c(0,0,0,0,0,0,0,0,0,0,0,0,0,0)$

med2. $r<-$ mean (sm3frutram2)

$\operatorname{med} 2 . r$

$\operatorname{var} 2 . r<-\operatorname{var}($ sm3frutram2)

$\operatorname{var} 2 . r$

\# Média geral e média das variâncias.

p.r<-(med1.r+med2.r) $/ 2$

p.r

medvar.r<-( $\operatorname{var} 1 . r+\operatorname{var} 2 . r) / 2$

medvar.r

\# Média e variância para a distribuição binomial.

$\mathrm{np} . \mathrm{r}<-10 * \mathrm{p} . \mathrm{r}$

np.r

npq. $r<-10 *$ p.r*(1-p.r)

npq.r

\# Aplicação do teste de qui-quadrado para verificar a eficiência do ajuste da distribuição binomial aos dados de presença-ausência. nmed1.np. 2.r<- ((10*med1.r) $-(\mathrm{np} . \mathrm{r}))^{\wedge} 2$

nmed2.np. 2.r<-((10*med2.r) $-(n p \cdot r))^{\wedge} 2$

ajmedsm3.r<-(nmed1.np.2.r+nmed2.np.2.r)/np.r

ajmedsm3.r

\#\# Frutos \#\# 
\# Primeira data de amostragem (07/08/2003).

sm3frutfrut $1<-c(0,0,0,0,0,0,0,0,0,0,0,0,0,0)$

med1.f<-mean (sm3frutfrut1)

$\operatorname{med} 1 . f$

$\operatorname{var} 1 . \mathrm{f}<-\operatorname{var}(\mathrm{sm} 3 \mathrm{frutfrut} 1)$

$\operatorname{var} 1 . f$

\# Segunda data de amostragem (19/09/2003).

sm3frutfrut $2<-c(0,0,0,0,0,0,0,0,0,0,0,0,0,0)$

med2.f<-mean (sm3frutfrut2)

$\operatorname{med} 2 . f$

$\operatorname{var} 2 . \mathrm{f}<-\operatorname{var}($ sm3frutfrut2)

$\operatorname{var} 2 . f$

\# Média geral e média das variâncias.

p.f<-(med1.f+med2.f $) / 2$

p.f

medvar.f<- $(\operatorname{var} 1 . f+\operatorname{var} 2 . f) / 2$

medvar.f

\# Média e variância para a distribuição binomial.

$\mathrm{np} . \mathrm{f}<-10 * \mathrm{p} . \mathrm{f}$

$\mathrm{np} . \mathrm{f}$

npq.f<- $10 *$ p.f*(1-p.f)

npq. $f$

\# Aplicação do teste de qui-quadrado para verificar a eficiência do ajuste da distribuição binomial aos dados de presença-ausência. nmed1.np.2.f<-((10*med1.f)-(np.f) )^2

nmed2.np. $2 . f<-((10 * \operatorname{med} 2 . f)-(\mathrm{np} . \mathrm{f}))^{\wedge} 2$

ajmedsm3.f<-(nmed1.np.2.f+nmed2.np.2.f $) / n p . f$

ajmedsm3.f 
\# Valor de qui-quadrado tabelado, com 1 (um) grau de liberdade e com nível de significância igual a 0,05.

qui. 2. tab. $1<-3.841$

qui.2.tab.1

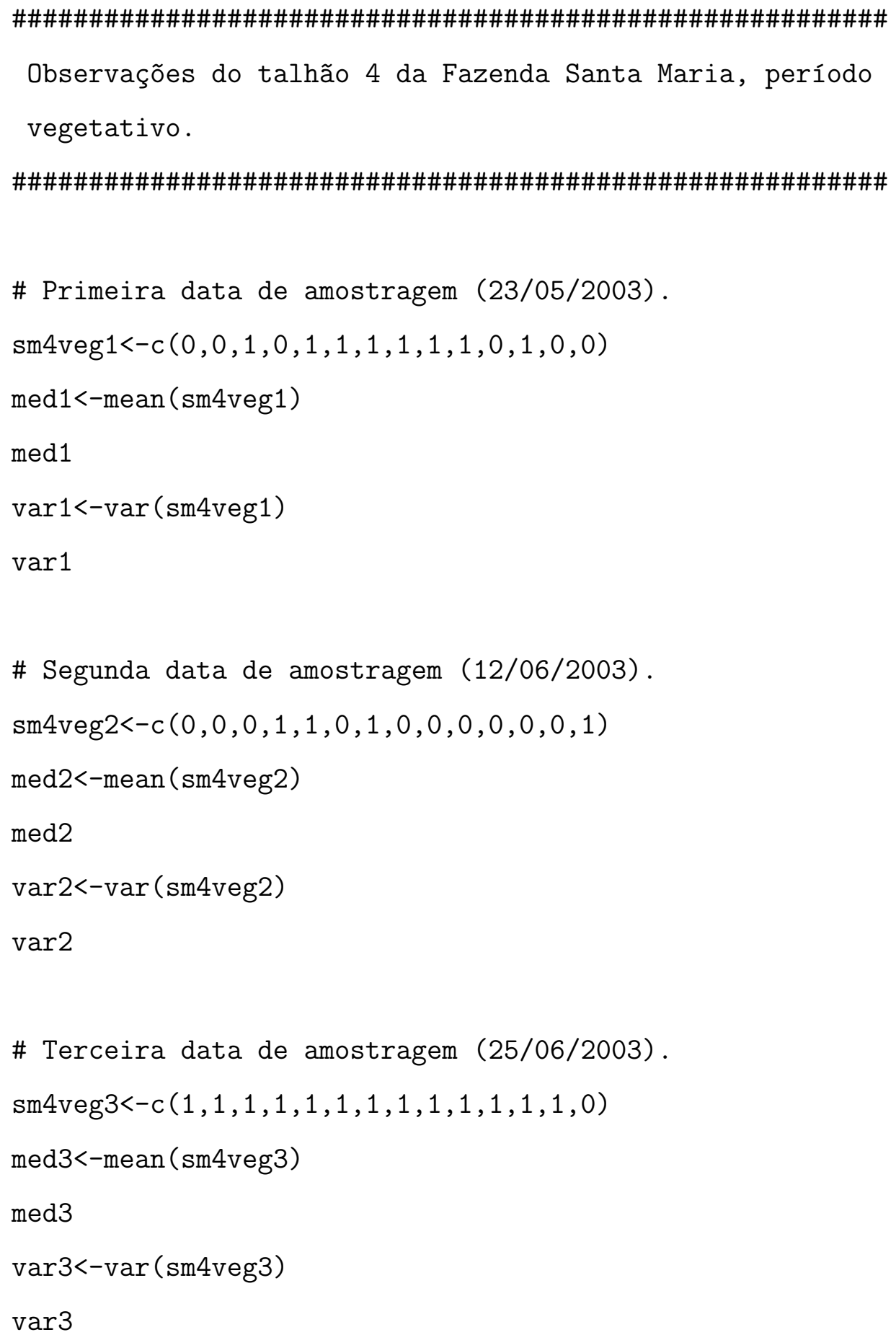


\# Quarta data de amostragem (11/07/2003).

sm4veg4<-c $(1,1,0,1,0,0,0,0,1,0,0,1,0,1)$

med4<-mean ( $\operatorname{sm} 4$ veg4)

med4

$\operatorname{var} 4<-\operatorname{var}(\operatorname{sm} 4 \operatorname{veg} 4)$

$\operatorname{var} 4$

\# Quinta data de amostragem (24/07/2003).

sm4veg5<-c $(1,0,0,1,0,1,0,0,1,0,1,0,0,0)$

med5<-mean (sm4veg5)

med5

$\operatorname{var} 5<-\operatorname{var}($ sm4veg5)

var5

\# Sexta data de amostragem (09/08/2003).

sm4veg6<-c $(1,0,0,0,1,0,0,0,0,1,0,0,0,1)$

med6<-mean (sm4veg6)

med6

$\operatorname{var} 6<-\operatorname{var}(\mathrm{sm} 4 \mathrm{veg} 6)$

$\operatorname{var} 6$

\# Média geral e média das variâncias.

$\mathrm{p}<-(\operatorname{med} 1+\operatorname{med} 2+\operatorname{med} 3+\operatorname{med} 4+\operatorname{med} 5+\operatorname{med} 6) / 6$

$\mathrm{p}$

medvar<-( $\operatorname{var} 1+\operatorname{var} 2+\operatorname{var} 3+\operatorname{var} 4+\operatorname{var} 5+\operatorname{var} 6) / 6$

medvar

\# Média e variância para a distribuição binomial.

$\mathrm{np}<-14 * \mathrm{p}$

$\mathrm{np}$

$\mathrm{npq}<-14 * \mathrm{p} *(1-\mathrm{p})$

$\mathrm{npq}$ 
\# Aplicação do teste de qui-quadrado para verificar a eficiência do ajuste da distribuição binomial aos dados de presença-ausência. nmed1.np. $2<-((14 * \operatorname{med} 1)-(\mathrm{np})))^{\wedge}$ nmed2.np. $2<-((14 * \operatorname{med} 2)-(\mathrm{np}))^{\wedge} 2$ nmed3.np. $2<-((14 * \operatorname{med} 3)-(\mathrm{np})))^{\wedge}$ nmed4.np. $2<-((14 * \operatorname{med} 4)-(\mathrm{np}))^{\wedge} 2$ nmed5.np. $2<-((14 * \text { med5 })-(\mathrm{np}))^{\wedge} 2$ nmed6.np. $2<-((14 * \operatorname{med} 6)-(\mathrm{np})))^{\wedge}$ ajmedsm4<-(nmed1.np. 2+nmed2.np. 2+nmed3.np. 2+nmed4.np. 2+nmed5.np. 2+nmed6.np. 2)/np ajmedsm4

\# Valor de qui-quadrado tabelado, com 5 (cinco) graus de liberdade e com nível de significância igual a 0,05.

qui. 2. tab. $5<-11.070$

qui.2.tab. 5 
ANEXO B - Construção das linhas de decisão dos planos de amostragem seqüencial, com níveis de erros iguais a 0,20 .

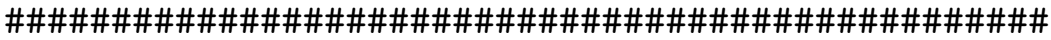 \\ Para amostragem em ramos no período vegetativo.

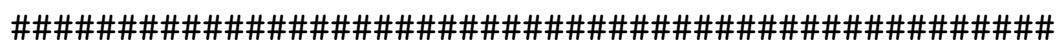

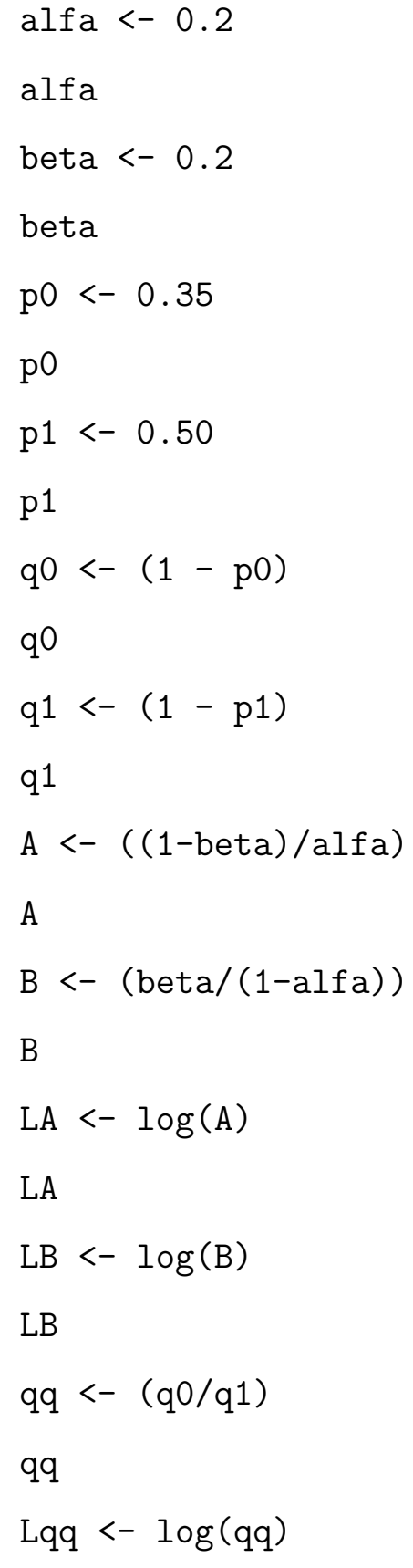




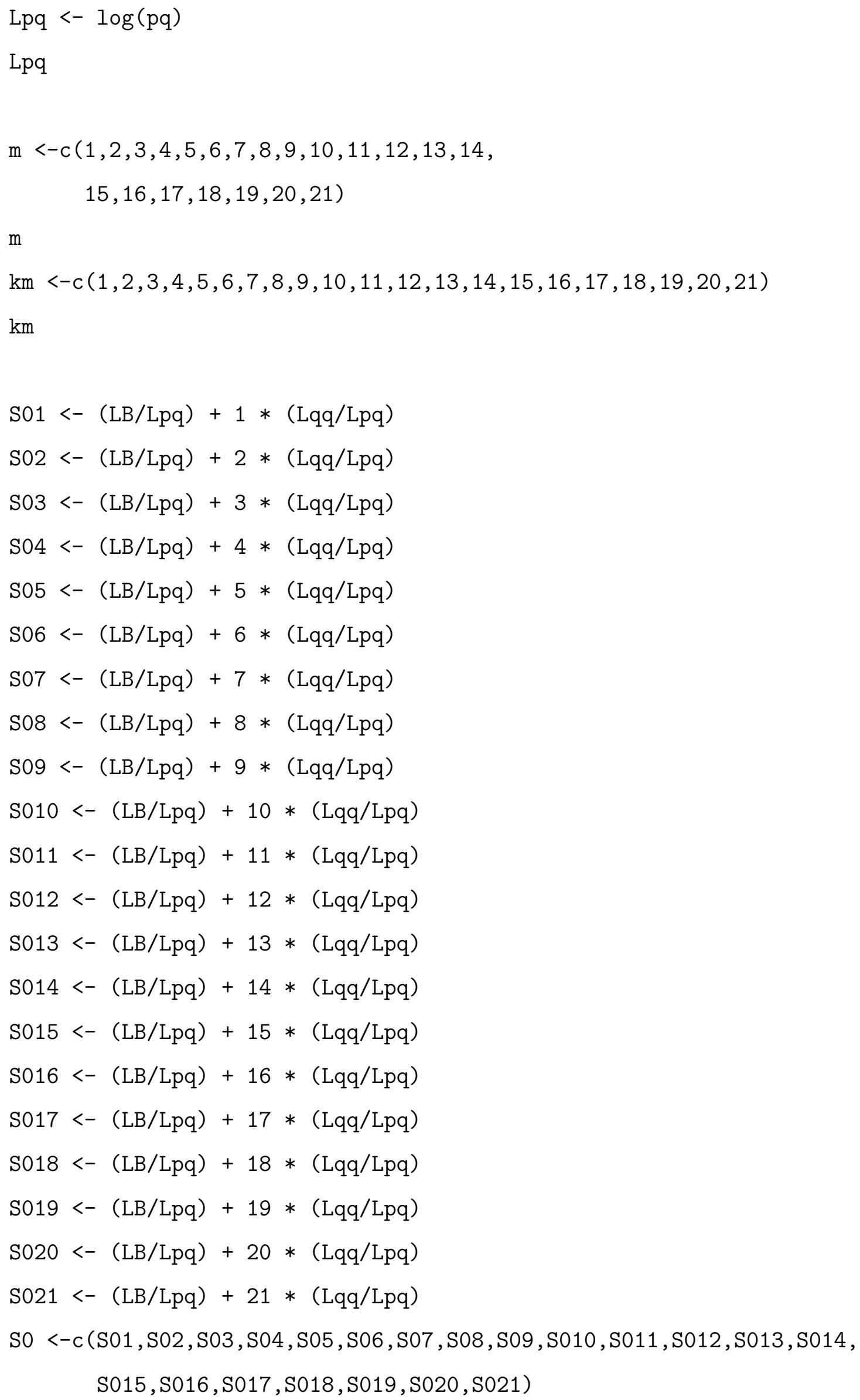


so

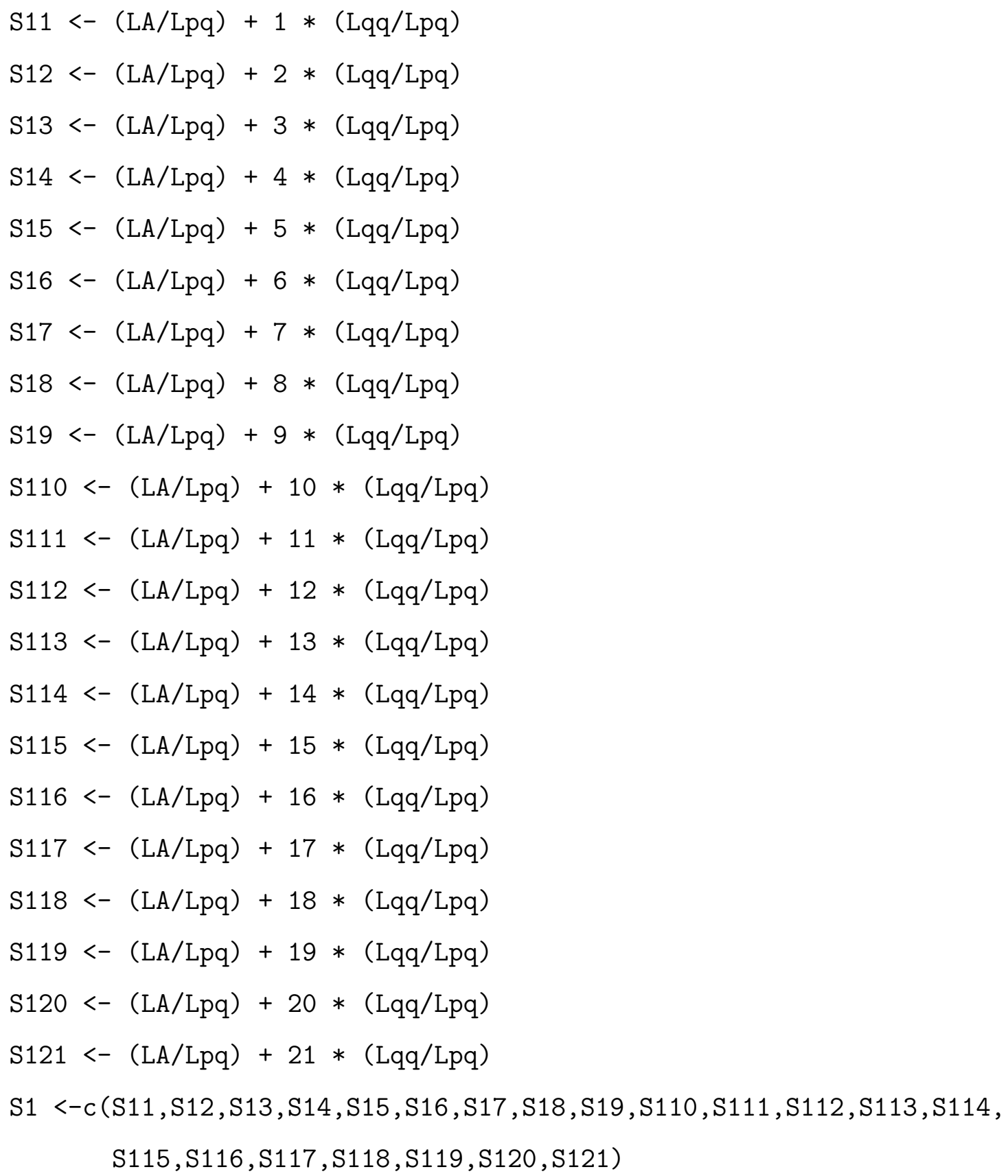

S1

$\operatorname{plot}(m, k m$, type $=" n ")$

lines $(\mathrm{m}, \mathrm{S} 0)$

lines $(m, S 1)$

text (c $(20.5,20.5), c(7.5,12.0), c(\operatorname{expression}(\mathrm{S}[0], \mathrm{S}[1])))$ 


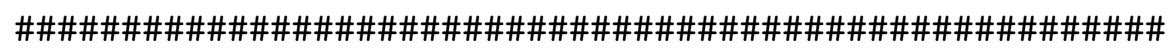

Para amostragem em ramos no período de frutificação.

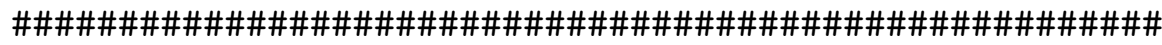

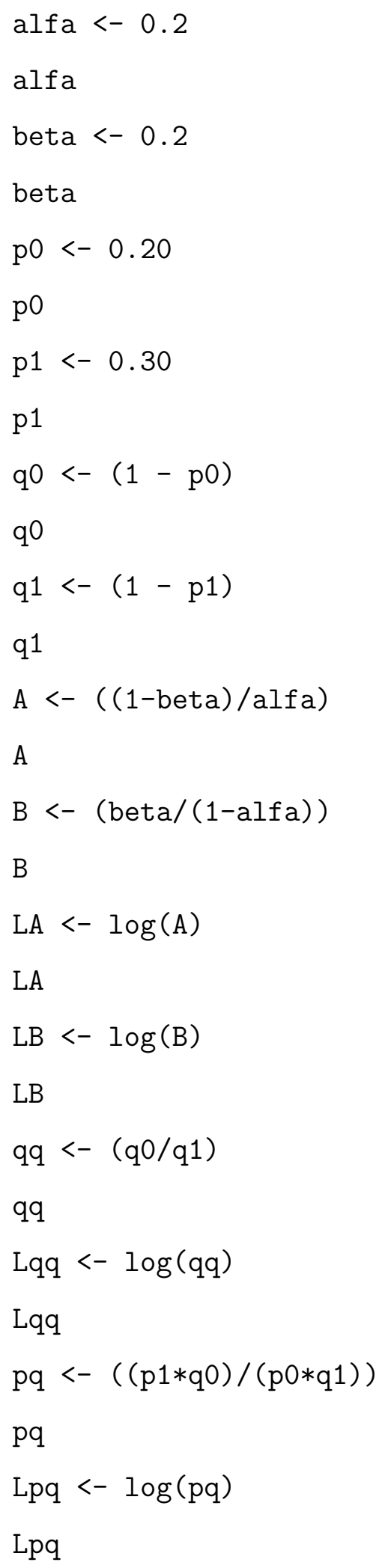


$\mathrm{m}<-c(1,2,3,4,5,6,7,8,9,10,11,12,13,14$,
$15,16,17,18,19,20,21,22,23,24,25$,
$26,27,28,29,30,31,32,33,34,35,36)$

$\mathrm{m}$

$\mathrm{km}<-c(0.4,0.8,1.2,1.6,2.0,2.4,2.8,3.2,3.6,4.0,4.4,4.8,5.2,5.4,5.8$, $6.2,6.4,6.8,7.2,7.6,8.0,8.4,8.8,9.2,9.6,10.0,10.4,10.8,11.2$, $11.6,12.0,12.4,12.8,13.2,13.6,14.0)$

$\mathrm{km}$

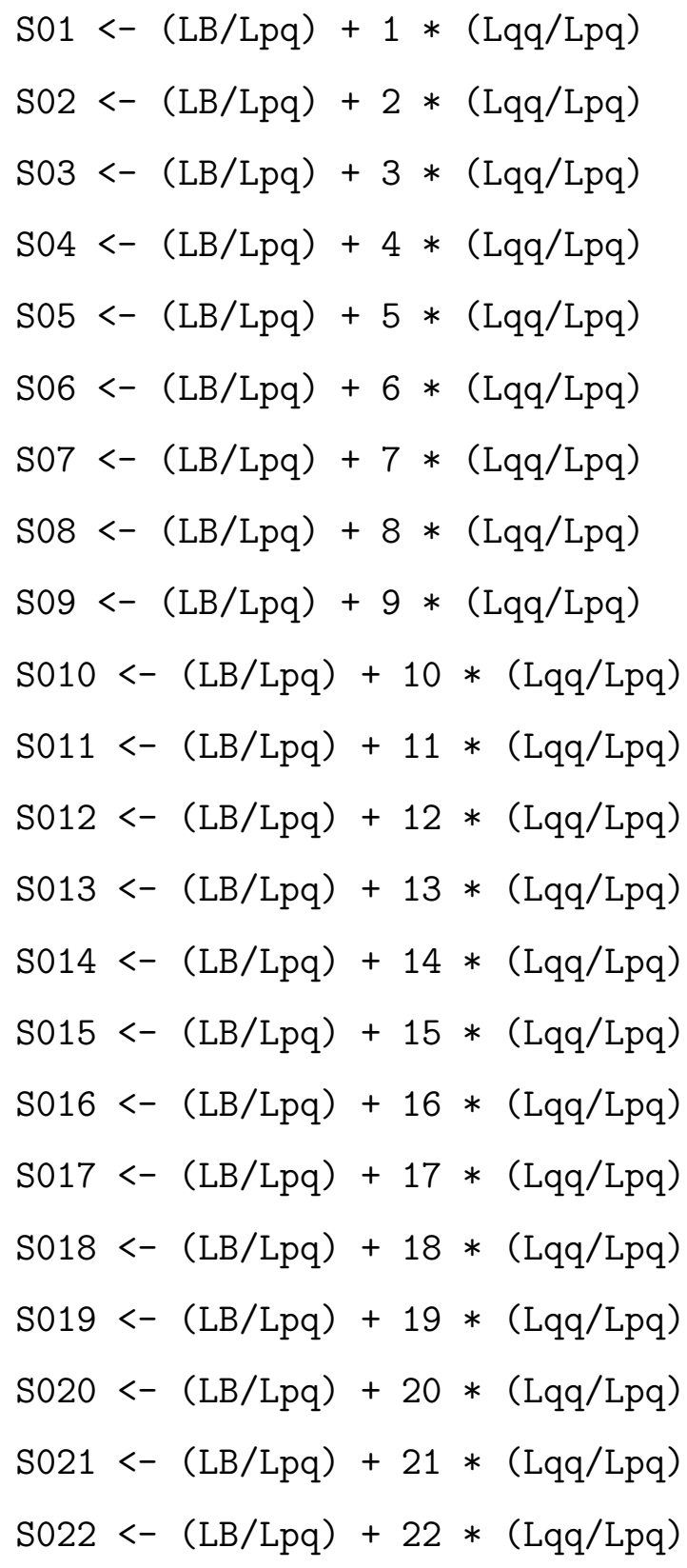




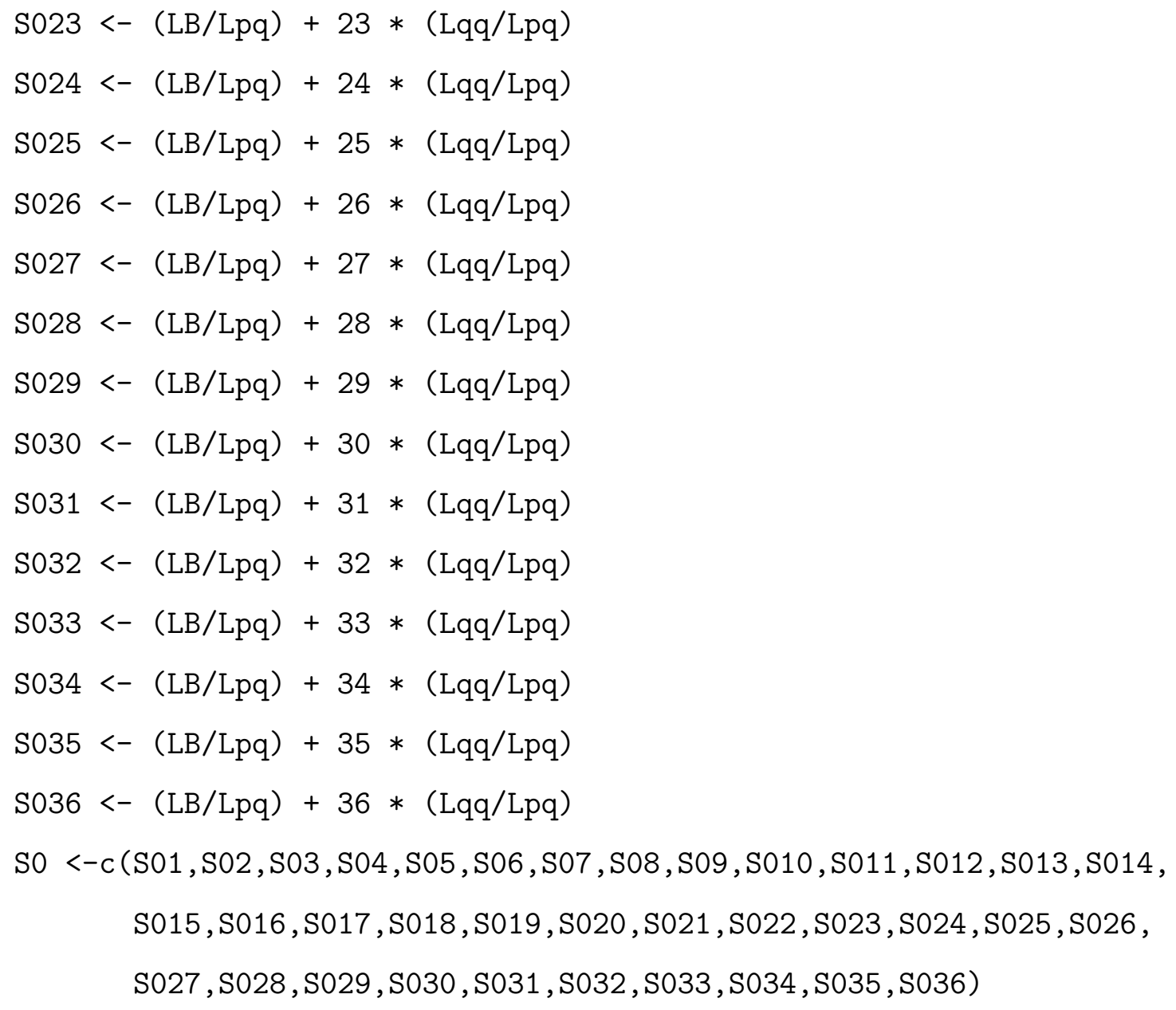

So

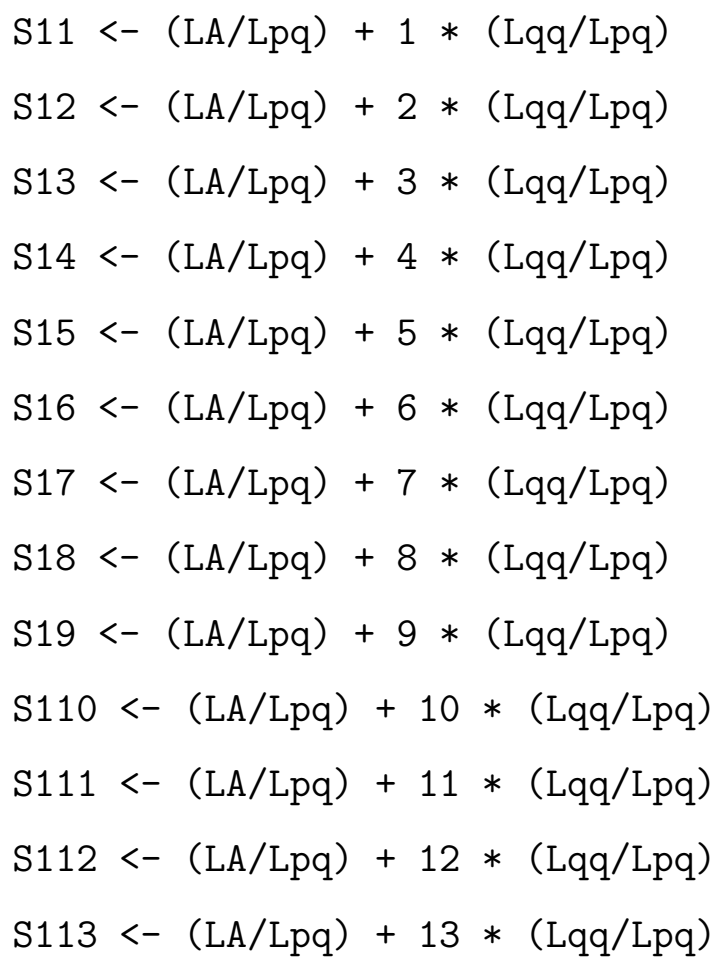




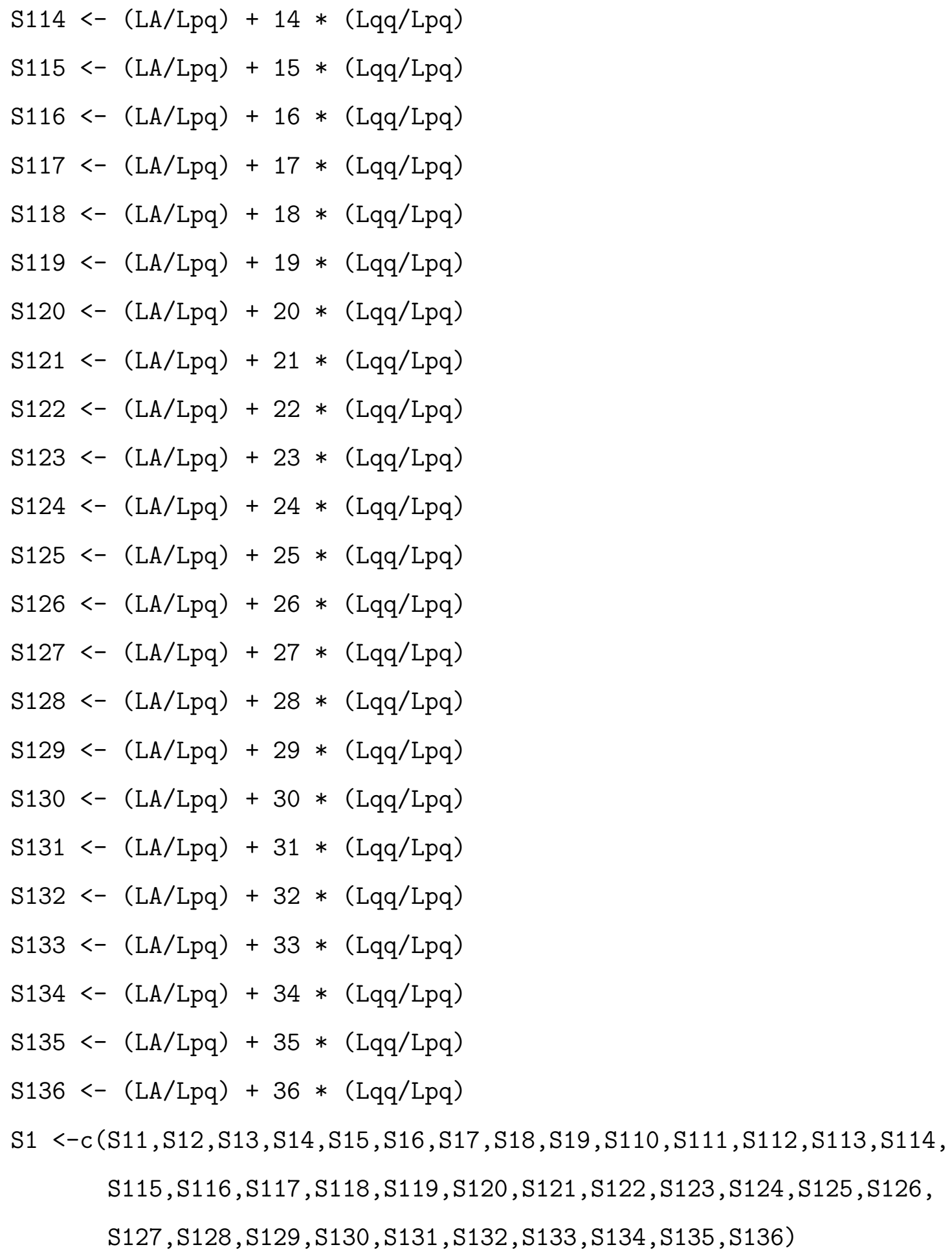

S1

plot $(m, k m$, type $=" n ")$

$\operatorname{lines}(\mathrm{m}, \mathrm{S} 0)$

lines $(m, S 1)$

$\operatorname{text}(c(35.5,35.5), c(7.0,12.0), c($ expression $(S[0], S[1])))$ 


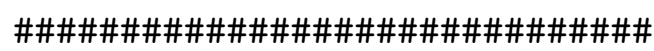

Para amostragem em frutos.

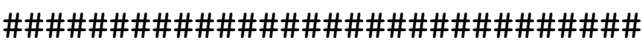

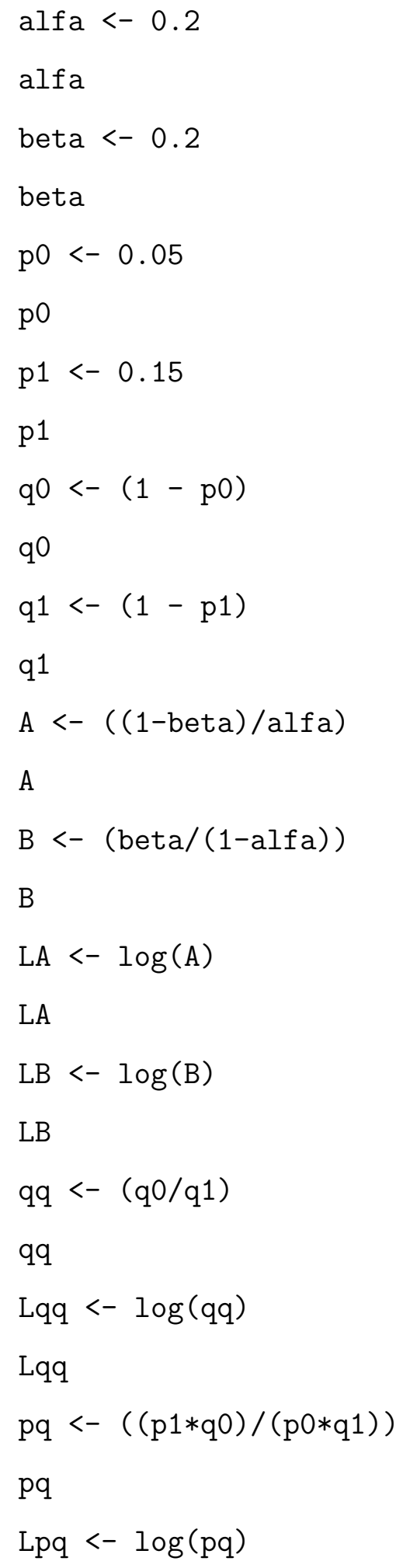




\section{Lpq}

$\mathrm{m}<-c(1,2,3,4,5,6,7,8,9,10,11,12,13,14,15,16,17)$

$\mathrm{m}$

$\mathrm{km}<-c(0.3,0.6,0.9,1.2,1.5,1.8,2.1,2.4,2.7,3.0,3.3,3.6$, $3.9,4.2,4.5,4.8,5.1)$

$\mathrm{km}$

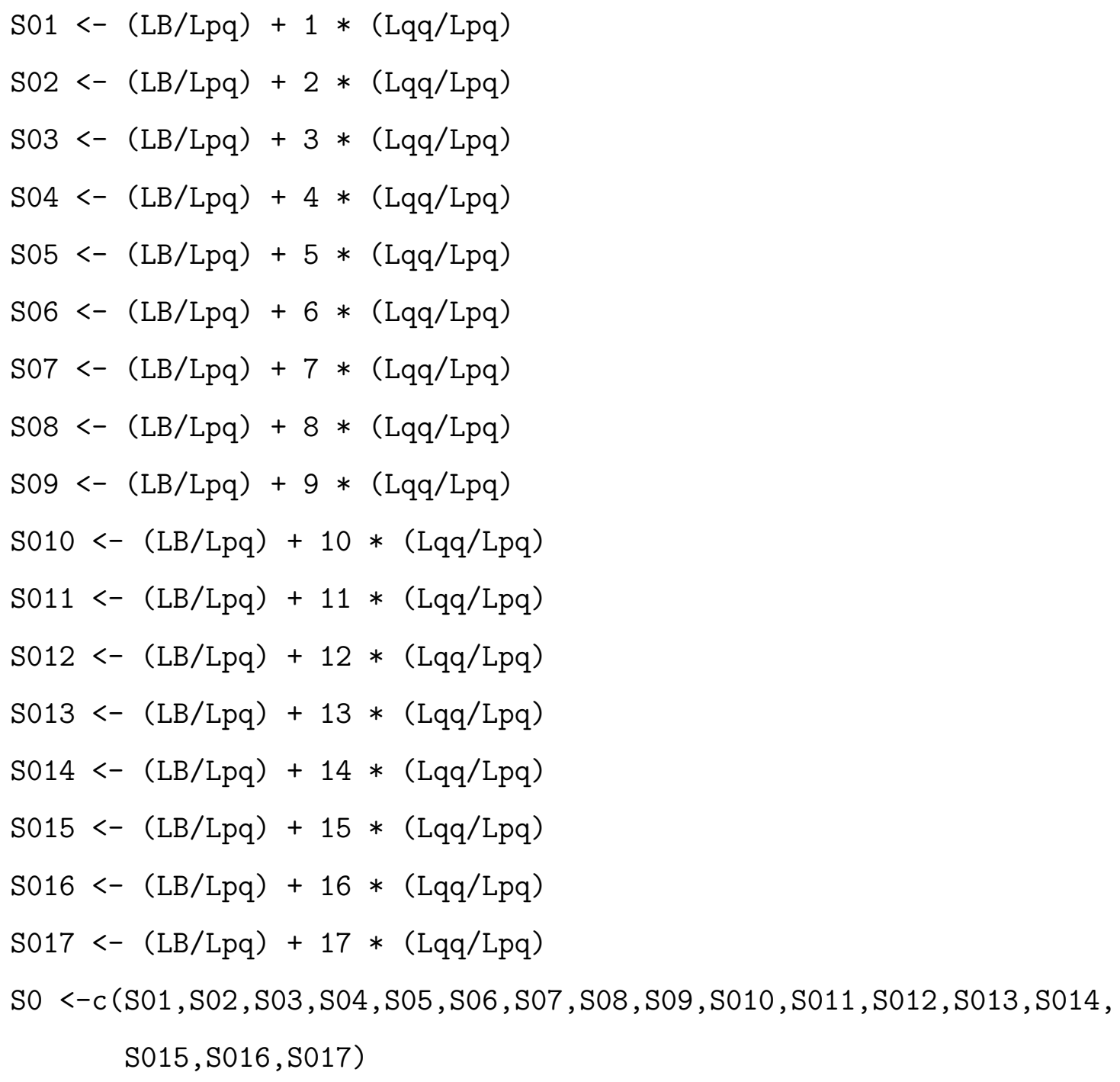




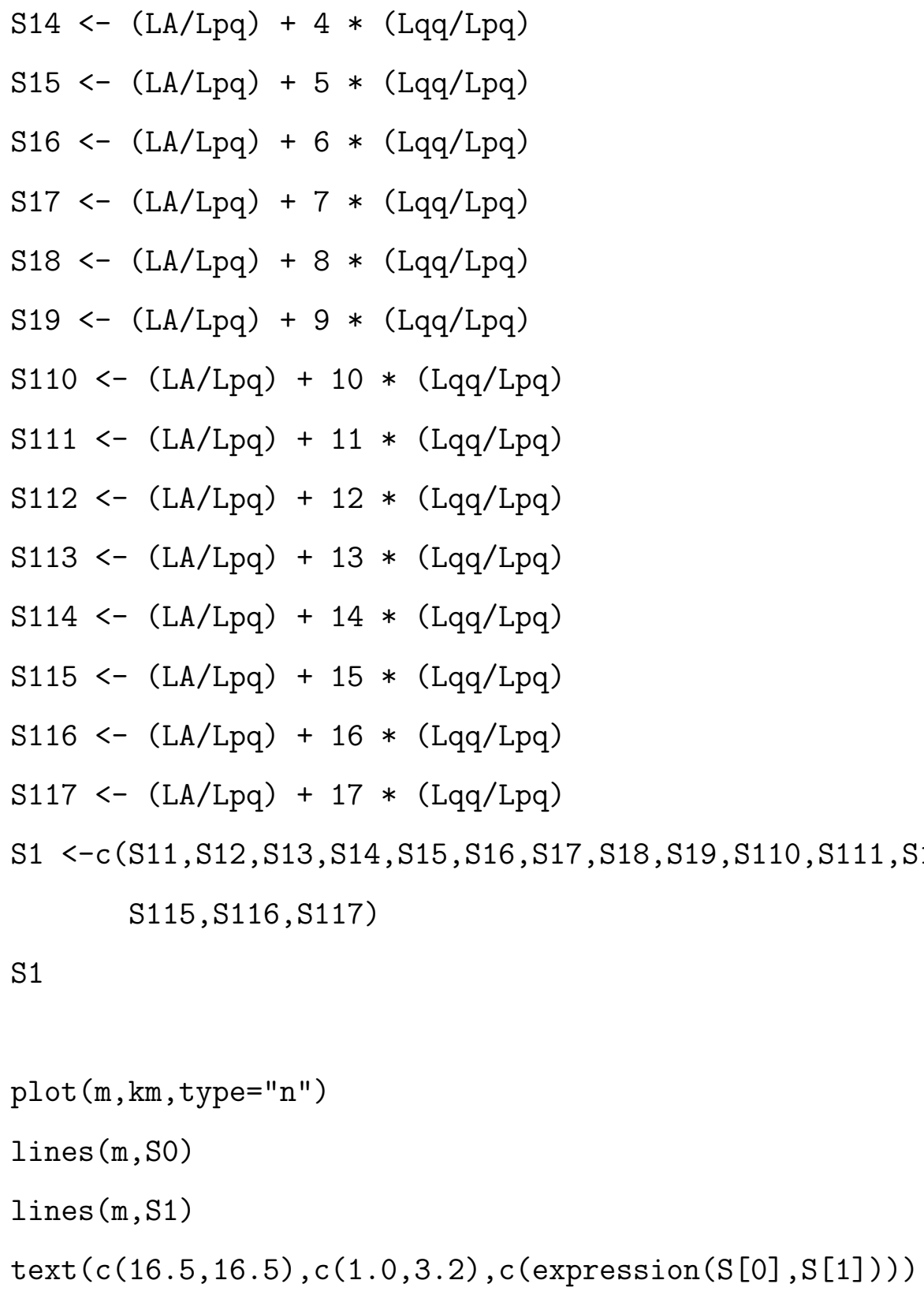


ANEXO C - Construção das linhas de decisão dos planos de amostragem seqüencial, com níveis de erros iguais a 0,10 .

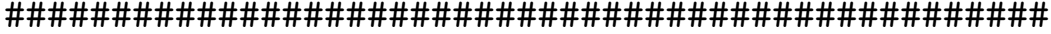 \\ Para amostragem em ramos no período vegetativo.

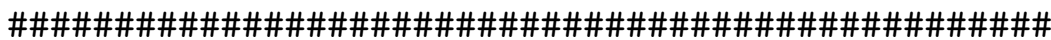

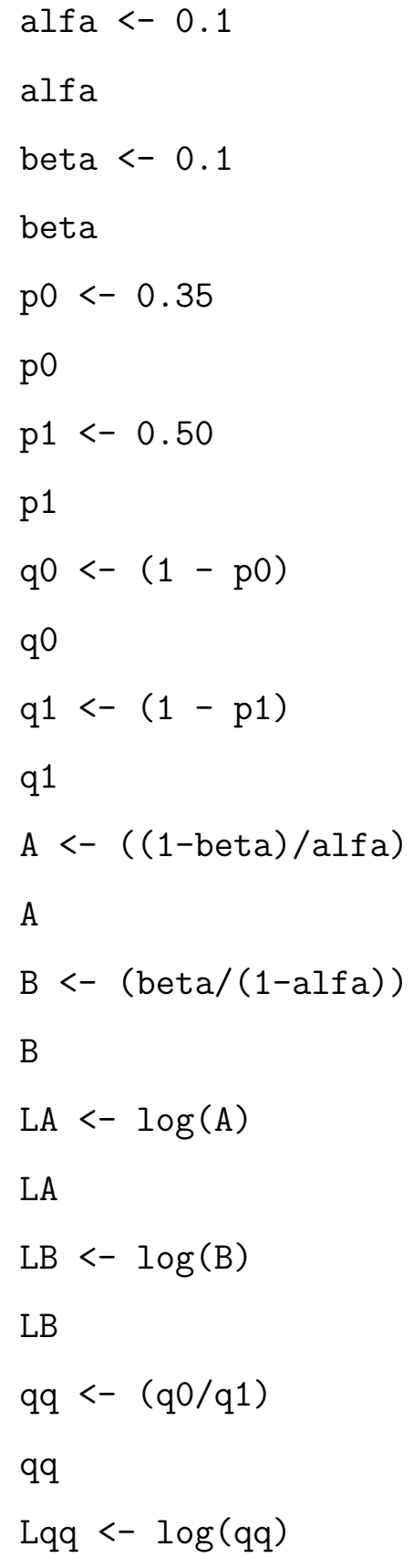


$\operatorname{Lpq}<-\log (\mathrm{pq})$

Lpq

$\mathrm{m}<-c(1,2,3,4,5,6,7,8,9,10,11,12,13,14$, $15,16,17,18,19,20,21,22,23,24,25,26$, $27,28,29,30,31,32,33,34,35,36,37,38$, $39,40,41,42,43,44,45,46,47,48,49,50$, $51,52)$

$\mathrm{m}$

$\mathrm{km}<-c(0.6,1.2,1.8,2.4,3.0,3.6,4.2,4.8,5.4,6.0,6.6,7.2,7.8$, 8.4, 9.0,9.6,10.2,10.8,11.4,12.0,12.6,13.2,13.8,14.4, 15.0,15.6,16.2 16.8,17.4,18.0,18.6,19.2,19.8, 20.4,21.0, $21.6,22.2,22.8,23.4,24.0,24.6,25.2,25.8,26.4,27.0,27.6$, $28.2,28.8,29.4,30.0,30.6,31.2)$

$\mathrm{km}$

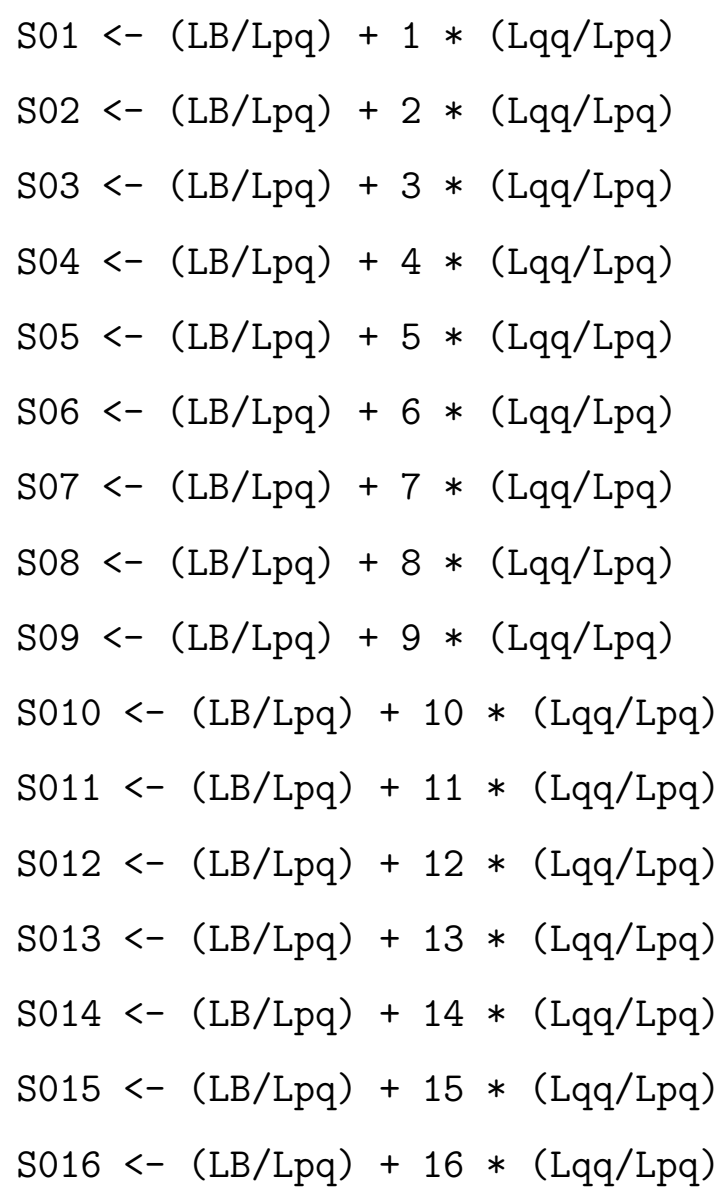




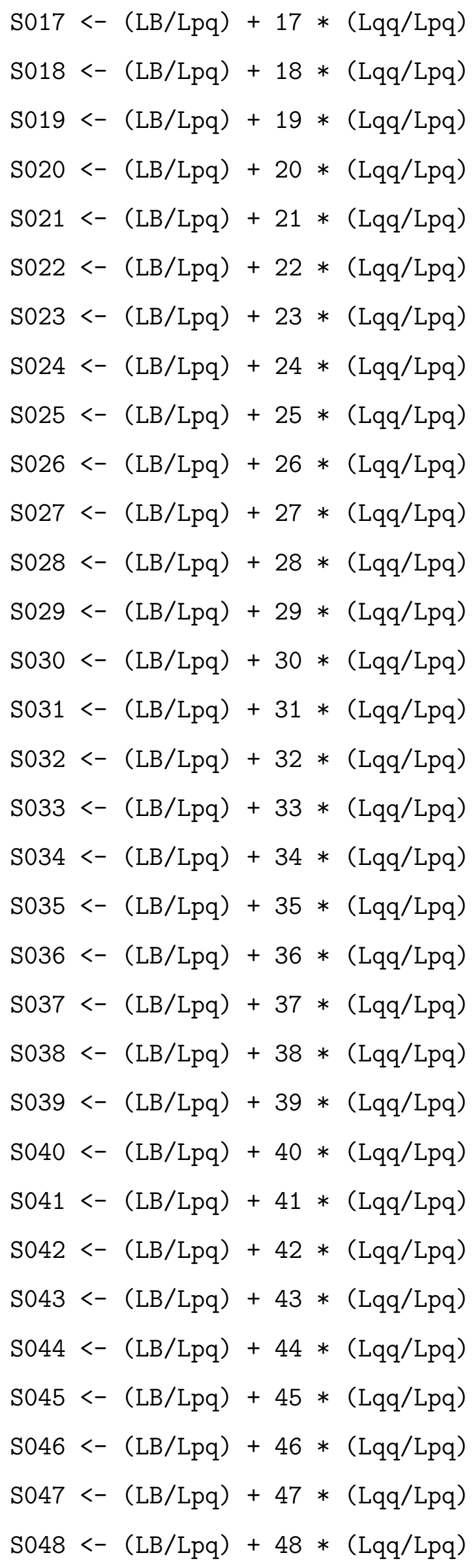




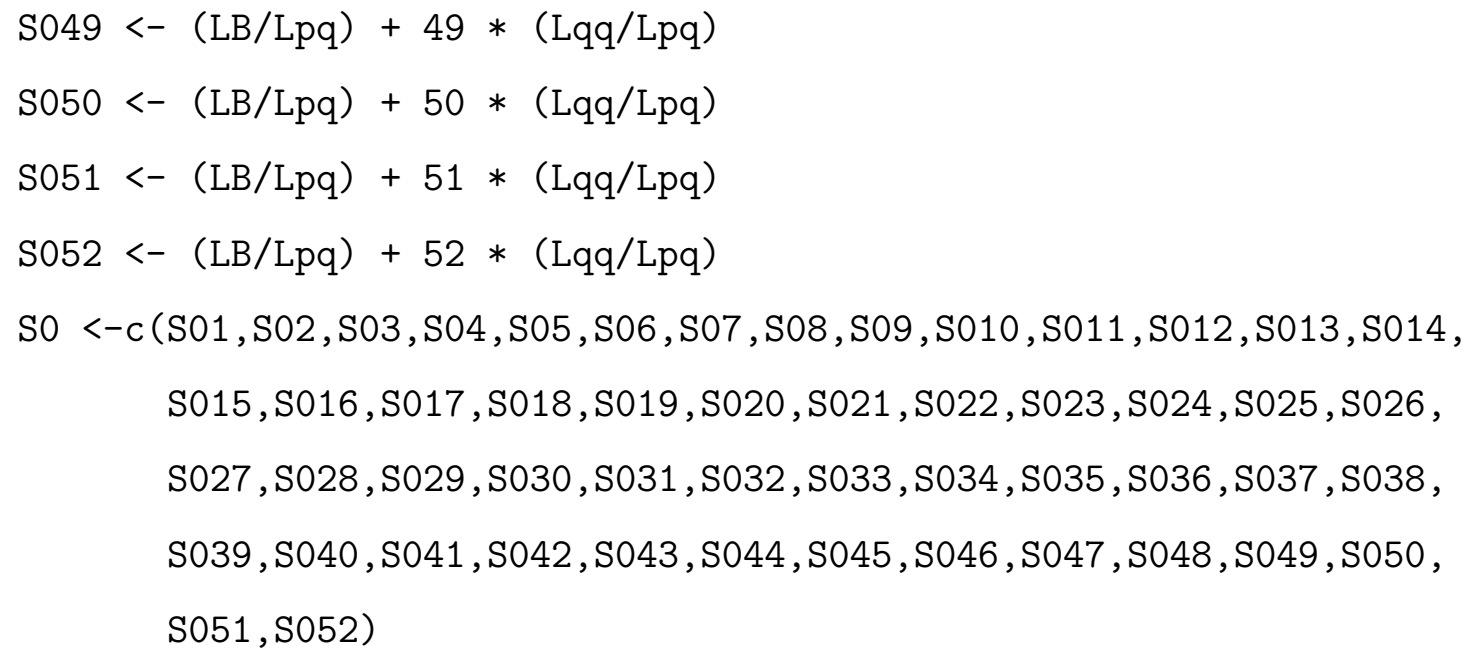

S0

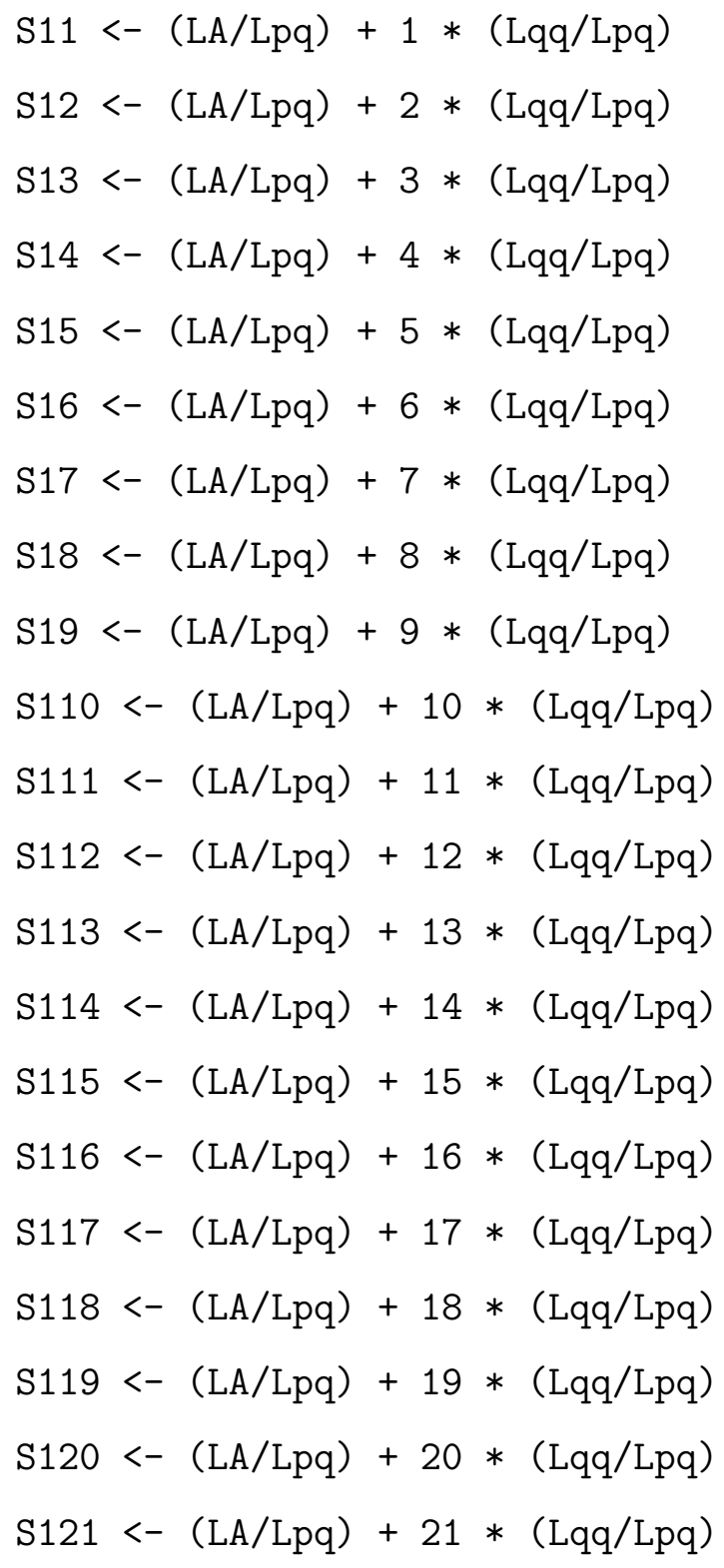




$$
\begin{aligned}
& \mathrm{S} 122<-(\mathrm{LA} / \mathrm{Lpq})+22 *(\mathrm{Lqq} / \mathrm{Lpq}) \\
& \mathrm{S} 123<-(\mathrm{LA} / \mathrm{Lpq})+23 *(\mathrm{Lqq} / \mathrm{Lpq}) \\
& \mathrm{S} 124<-(\mathrm{LA} / \mathrm{Lpq})+24 *(\mathrm{Lqq} / \mathrm{Lpq}) \\
& \mathrm{S} 125<-(\mathrm{LA} / \mathrm{Lpq})+25 *(\mathrm{Lqq} / \mathrm{Lpq}) \\
& \mathrm{S} 126<-(\mathrm{LA} / \mathrm{Lpq})+26 *(\mathrm{Lqq} / \mathrm{Lpq}) \\
& \mathrm{S} 127<-(\mathrm{LA} / \mathrm{Lpq})+27 *(\mathrm{Lqq} / \mathrm{Lpq}) \\
& \mathrm{S} 128<-(\mathrm{LA} / \mathrm{Lpq})+28 *(\mathrm{Lqq} / \mathrm{Lpq}) \\
& \mathrm{S} 129<-(\mathrm{LA} / \mathrm{Lpq})+29 *(\mathrm{Lqq} / \mathrm{Lpq}) \\
& \mathrm{S} 130<-(\mathrm{LA} / \mathrm{Lpq})+30 *(\mathrm{Lqq} / \mathrm{Lpq}) \\
& \mathrm{S} 131<-(\mathrm{LA} / \mathrm{Lpq})+31 *(\mathrm{Lqq} / \mathrm{Lpq}) \\
& \mathrm{S} 132<-(\mathrm{LA} / \mathrm{Lpq})+32 *(\mathrm{Lqq} / \mathrm{Lpq}) \\
& \mathrm{S} 133<-(\mathrm{LA} / \mathrm{Lpq})+33 *(\mathrm{Lqq} / \mathrm{Lpq}) \\
& \mathrm{S} 134<-(\mathrm{LA} / \mathrm{Lpq})+34 *(\mathrm{Lqq} / \mathrm{Lpq}) \\
& \mathrm{S} 135<-(\mathrm{LA} / \mathrm{Lpq})+35 *(\mathrm{Lqq} / \mathrm{Lpq}) \\
& \mathrm{S} 136<-(\mathrm{LA} / \mathrm{Lpq})+36 *(\mathrm{Lqq} / \mathrm{Lpq}) \\
& \mathrm{S} 137<-(\mathrm{LA} / \mathrm{Lpq})+37 *(\mathrm{Lqq} / \mathrm{Lpq}) \\
& \mathrm{S} 138<-(\mathrm{LA} / \mathrm{Lpq})+38 *(\mathrm{Lqq} / \mathrm{Lpq}) \\
& \mathrm{S} 139<-(\mathrm{LA} / \mathrm{Lpq})+39 *(\mathrm{Lqq} / \mathrm{Lpq}) \\
& \mathrm{S} 140<-(\mathrm{LA} / \mathrm{Lpq})+40 *(\mathrm{Lqq} / \mathrm{Lpq}) \\
& \mathrm{S} 141<-(\mathrm{LA} / \mathrm{Lpq})+41 *(\mathrm{Lqq} / \mathrm{Lpq}) \\
& \mathrm{S} 142<-(\mathrm{LA} / \mathrm{Lpq})+42 *(\mathrm{Lqq} / \mathrm{Lpq}) \\
& \mathrm{S} 143<-(\mathrm{LA} / \mathrm{Lpq})+43 *(\mathrm{Lqq} / \mathrm{Lpq}) \\
& \mathrm{S} 144<-(\mathrm{LA} / \mathrm{Lpq})+44 *(\mathrm{Lqq} / \mathrm{Lpq}) \\
& \mathrm{S} 145<-(\mathrm{LA} / \mathrm{Lpq})+45 *(\mathrm{Lqq} / \mathrm{Lpq}) \\
& \mathrm{S} 146<-(\mathrm{LA} / \mathrm{Lpq})+46 *(\mathrm{Lqq} / \mathrm{Lpq}) \\
& \mathrm{S} 147<-(\mathrm{LA} / \mathrm{Lpq})+47 *(\mathrm{Lqq} / \mathrm{Lpq}) \\
& \mathrm{S} 148<-(\mathrm{LA} / \mathrm{Lpq})+48 *(\mathrm{Lqq} / \mathrm{Lpq}) \\
& \mathrm{S} 149<-(\mathrm{LA} / \mathrm{Lpq})+49 *(\mathrm{Lqq} / \mathrm{Lpq}) \\
& \mathrm{S} 150<-(\mathrm{LA} / \mathrm{Lpq})+50 *(\mathrm{Lqq} / \mathrm{Lpq}) \\
& \mathrm{S} 151<-(\mathrm{LA} / \mathrm{Lpq})+51 *(\mathrm{Lqq} / \mathrm{Lpq}) \\
& \mathrm{S} 152<-(\mathrm{LA} / \mathrm{Lpq})+52 *(\mathrm{Lqq} / \mathrm{Lpq})
\end{aligned}
$$

$\mathrm{S} 1<-\mathrm{c}(\mathrm{S} 11, \mathrm{~S} 12, \mathrm{~S} 13, \mathrm{~S} 14, \mathrm{~S} 15, \mathrm{~S} 16, \mathrm{~S} 17, \mathrm{~S} 18, \mathrm{~S} 19$, S110 , S111 , S112 , S113, S114, 
$\mathrm{S} 115, \mathrm{~S} 116, \mathrm{~S} 117, \mathrm{~S} 118, \mathrm{~S} 119$, S120 , S121 , S122 , S123, S124, S125, S126, $\mathrm{S} 127, \mathrm{~S} 128, \mathrm{~S} 129, \mathrm{~S} 130, \mathrm{~S} 131, \mathrm{~S} 132, \mathrm{~S} 133, \mathrm{~S} 134, \mathrm{~S} 135, \mathrm{~S} 136, \mathrm{~S} 137, \mathrm{~S} 138$, $\mathrm{S} 139, \mathrm{~S} 140, \mathrm{~S} 141, \mathrm{~S} 142, \mathrm{~S} 143, \mathrm{~S} 144, \mathrm{~S} 145, \mathrm{~S} 146, \mathrm{~S} 147$, S148, S149, S150, $\mathrm{S} 151, \mathrm{~S} 152)$

S1

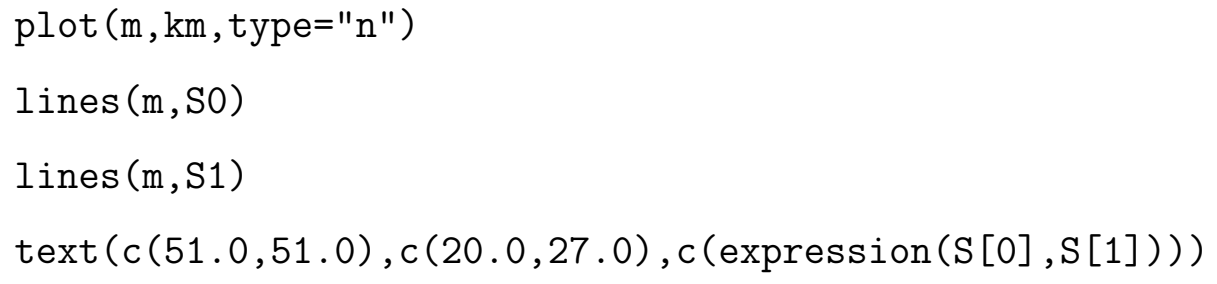

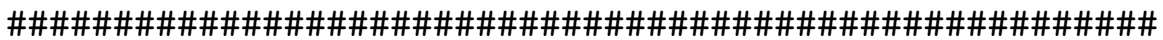

Para amostragem em ramos no período de frutificação.

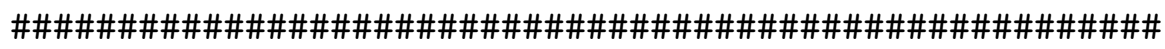

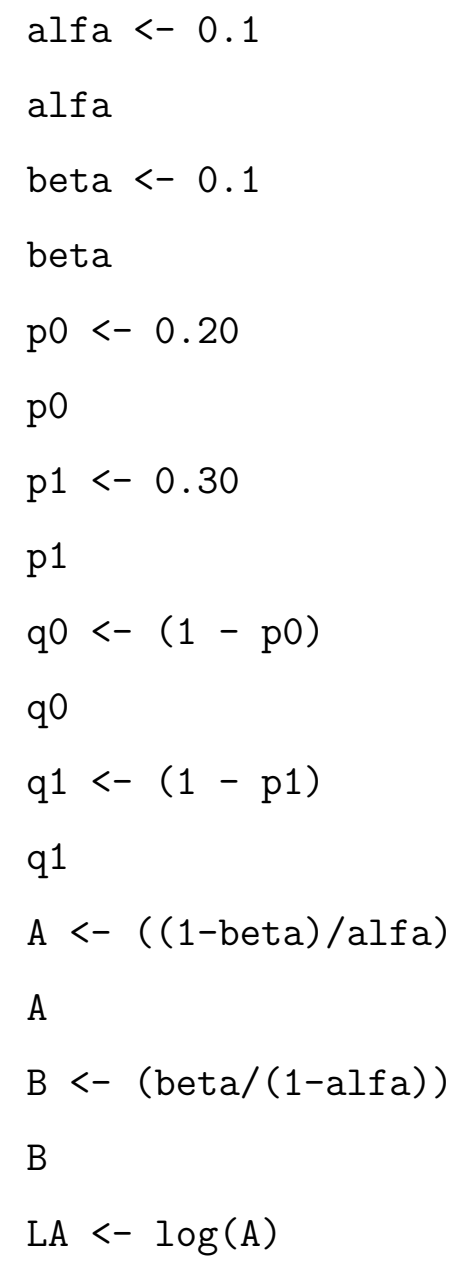


LA

$\mathrm{LB}<-\log (B)$

LB

$q q<-(q 0 / q 1)$

qq

Lqq $<-\log (q q)$

Lqq

$p q<-((p 1 * q 0) /(p 0 * q 1))$

$\mathrm{pq}$

Lpq $<-\log (p q)$

Lpq

$\mathrm{m}<-c(1,2,3,4,5,6,7,8,9,10,11,12,13,14,15,16,17$, $18,19,20,21,22,23,24,25,26,27,28,29,30,31,32$, $33,34,35,36,37,38,39,40,41,42,43,44,45,46,47$, $48,49,50,51,52,53,54,55,56,57,58,59,60,61,62$, $63,64,65,66,67,68,69,70,71,72,73,74,75,76,77$, $78,79,80,81,82,83,84,85,86,87,88,89)$

$\mathrm{m}$

$\mathrm{km}<-c(0.4,0.8,1.2,1.6,2.0,2.4,2.8,3.2,3.6,4.0,4.4,4.8,5.2,5.6,6.0$, $6.4,6.8,7.2,7.6,8.0,8.4,8.8,9.2,9.6,10.0,10.4,10.8,11.2,11.6$, $12.0,12.4,12.8,13.2,13.6,14.0,14.4,14.8,15.2,15.6,16.0,16.4$, $16.8,17.2,17.6,18.0,18.4,18.8,19.2,19.6,20.0,20.4,20.8,21.2$, $21.6,22.0,22.4,22.8,23.2,23.6,24.0,24.4,24.8,25.2,25.6,26.0$, $26.4,26.8,27.2,27.6,28.0,28.4,28.8,29.2,29.6,30.0,30.4,30.8$, $31.2,31.6,32.0,32.4,32.8,33.2,33.6,34.0,34.4,34.8,35.2,35.6)$

$\mathrm{km}$

$\mathrm{S} 01<-(\mathrm{LB} / \mathrm{Lpq})+1 *(\mathrm{Lqq} / \mathrm{Lpq})$
$\mathrm{S} 02<-(\mathrm{LB} / \mathrm{Lpq})+2 *(\mathrm{Lqq} / \mathrm{Lpq})$
$\mathrm{S} 03<-(\mathrm{LB} / \mathrm{Lpq})+3 *(\mathrm{Lqq} / \mathrm{Lpq})$
$\mathrm{S} 04<-(\mathrm{LB} / \mathrm{Lpq})+4 *(\mathrm{Lqq} / \mathrm{Lpq})$ 


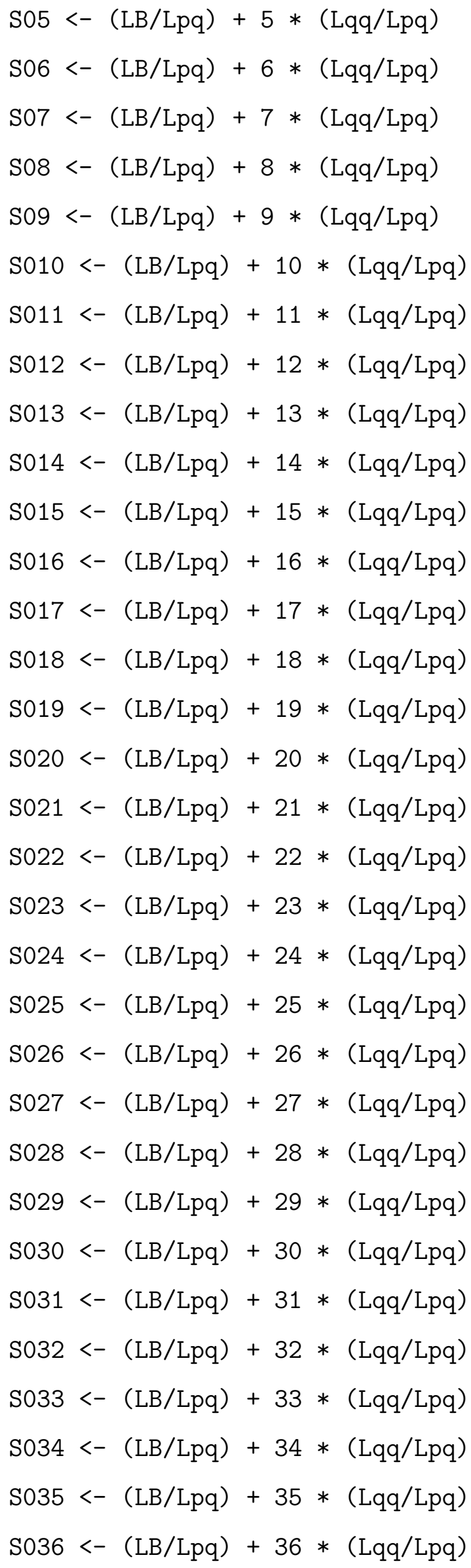




$$
\begin{aligned}
& \text { S037 <- (LB/Lpq) }+37 *(\mathrm{Lqq} / \mathrm{Lpq}) \\
& \mathrm{S} 038<-(\mathrm{LB} / \mathrm{Lpq})+38 *(\mathrm{Lqq} / \mathrm{Lpq}) \\
& \mathrm{S} 039<-(\mathrm{LB} / \mathrm{Lpq})+39 *(\mathrm{Lqq} / \mathrm{Lpq}) \\
& \mathrm{S} 040<-(\mathrm{LB} / \mathrm{Lpq})+40 *(\mathrm{Lqq} / \mathrm{Lpq}) \\
& \mathrm{S} 041<-(\mathrm{LB} / \mathrm{Lpq})+41 *(\mathrm{Lqq} / \mathrm{Lpq}) \\
& \mathrm{S} 042<-(\mathrm{LB} / \mathrm{Lpq})+42 *(\mathrm{Lqq} / \mathrm{Lpq}) \\
& \mathrm{S} 043<-(\mathrm{LB} / \mathrm{Lpq})+43 *(\mathrm{Lqq} / \mathrm{Lpq}) \\
& \mathrm{S} 044<-(\mathrm{LB} / \mathrm{Lpq})+44 *(\mathrm{Lqq} / \mathrm{Lpq}) \\
& \mathrm{S} 045<-(\mathrm{LB} / \mathrm{Lpq})+45 *(\mathrm{Lqq} / \mathrm{Lpq}) \\
& \text { S046 <- (LB/Lpq) }+46 *(L q q / L p q) \\
& \text { S047<- (LB/Lpq) }+47 *(L q q / L p q) \\
& \mathrm{S} 048<-(\mathrm{LB} / \mathrm{Lpq})+48 *(\mathrm{Lqq} / \mathrm{Lpq}) \\
& \mathrm{S} 049<-(\mathrm{LB} / \mathrm{Lpq})+49 *(\mathrm{Lqq} / \mathrm{Lpq}) \\
& \mathrm{S} 050<-(\mathrm{LB} / \mathrm{Lpq})+50 *(\mathrm{Lqq} / \mathrm{Lpq}) \\
& \mathrm{S} 051<-(\mathrm{LB} / \mathrm{Lpq})+51 *(\mathrm{Lqq} / \mathrm{Lpq}) \\
& \mathrm{S} 052<-(\mathrm{LB} / \mathrm{Lpq})+52 *(\mathrm{Lqq} / \mathrm{Lpq}) \\
& \text { S053<- (LB/Lpq })+53 *(L q q / L p q) \\
& \mathrm{S} 054<-(\mathrm{LB} / \mathrm{Lpq})+54 *(\mathrm{Lqq} / \mathrm{Lpq}) \\
& \mathrm{S} 055<-(\mathrm{LB} / \mathrm{Lpq})+55 *(\mathrm{Lqq} / \mathrm{Lpq}) \\
& \mathrm{S} 056<-(\mathrm{LB} / \mathrm{Lpq})+56 *(\mathrm{Lqq} / \mathrm{Lpq}) \\
& \text { S057<- (LB/Lpq) }+57 *(\mathrm{Lqq} / \mathrm{Lpq}) \\
& \mathrm{S} 058<-(\mathrm{LB} / \mathrm{Lpq})+58 *(\mathrm{Lqq} / \mathrm{Lpq}) \\
& \mathrm{S} 059<-(\mathrm{LB} / \mathrm{Lpq})+59 *(\mathrm{Lqq} / \mathrm{Lpq}) \\
& \mathrm{S} 060<-(\mathrm{LB} / \mathrm{Lpq})+60 *(\mathrm{Lqq} / \mathrm{Lpq}) \\
& \mathrm{S} 061<-(\mathrm{LB} / \mathrm{Lpq})+61 *(\mathrm{Lqq} / \mathrm{Lpq}) \\
& \mathrm{S} 062<-(\mathrm{LB} / \mathrm{Lpq})+62 *(\mathrm{Lqq} / \mathrm{Lpq}) \\
& \mathrm{S} 063<-(\mathrm{LB} / \mathrm{Lpq})+63 *(\mathrm{Lqq} / \mathrm{Lpq}) \\
& \mathrm{S} 064<-(\mathrm{LB} / \mathrm{Lpq})+64 *(\mathrm{Lqq} / \mathrm{Lpq}) \\
& \mathrm{S} 065<-(\mathrm{LB} / \mathrm{Lpq})+65 *(\mathrm{Lqq} / \mathrm{Lpq}) \\
& \mathrm{S} 066<-(\mathrm{LB} / \mathrm{Lpq})+66 *(\mathrm{Lqq} / \mathrm{Lpq}) \\
& \text { S067 <- (LB/Lpq })+67 *(L q q / L p q) \\
& \mathrm{S} 068<-(\mathrm{LB} / \mathrm{Lpq})+68 *(\mathrm{Lqq} / \mathrm{Lpq})
\end{aligned}
$$




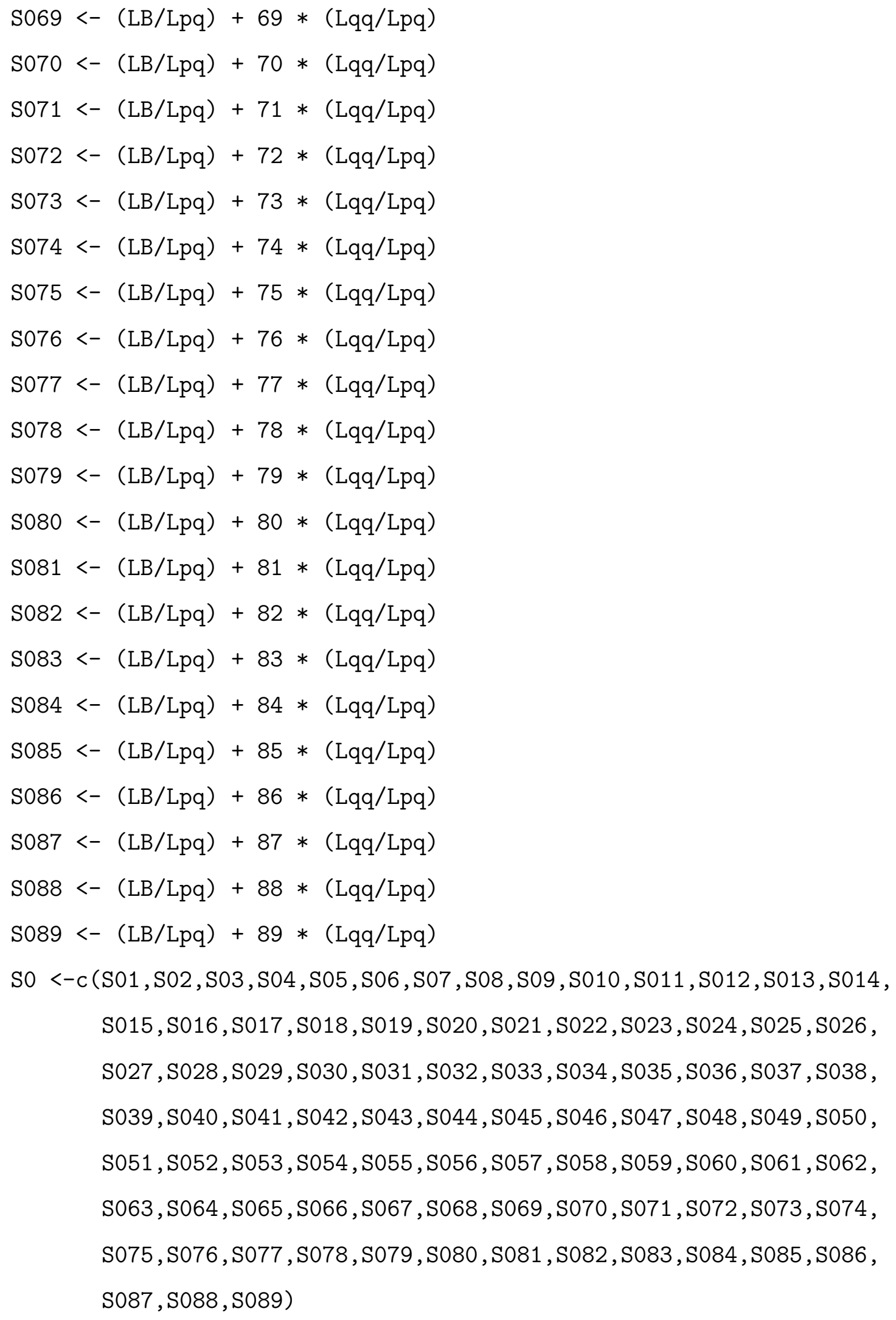




$$
\begin{aligned}
& \mathrm{S} 12<-(\mathrm{LA} / \mathrm{Lpq})+2 *(\mathrm{Lqq} / \mathrm{Lpq}) \\
& \mathrm{S} 13<-(\mathrm{LA} / \mathrm{Lpq})+3 *(\mathrm{Lqq} / \mathrm{Lpq}) \\
& \mathrm{S} 14<-(\mathrm{LA} / \mathrm{Lpq})+4 *(\mathrm{Lqq} / \mathrm{Lpq}) \\
& \mathrm{S} 15<-(\mathrm{LA} / \mathrm{Lpq})+5 *(\mathrm{Lqq} / \mathrm{Lpq}) \\
& \mathrm{S} 16<-(\mathrm{LA} / \mathrm{Lpq})+6 *(\mathrm{Lqq} / \mathrm{Lpq}) \\
& \mathrm{S} 17<-(\mathrm{LA} / \mathrm{Lpq})+7 *(\mathrm{Lqq} / \mathrm{Lpq}) \\
& \mathrm{S} 18<-(\mathrm{LA} / \mathrm{Lpq})+8 *(\mathrm{Lqq} / \mathrm{Lpq}) \\
& \mathrm{S} 19<-(\mathrm{LA} / \mathrm{Lpq})+9 *(\mathrm{Lqq} / \mathrm{Lpq}) \\
& \mathrm{S} 110<-(\mathrm{LA} / \mathrm{Lpq})+10 *(\mathrm{Lqq} / \mathrm{Lpq}) \\
& \mathrm{S} 111<-(\mathrm{LA} / \mathrm{Lpq})+11 *(\mathrm{Lqq} / \mathrm{Lpq}) \\
& \mathrm{S} 112<-(\mathrm{LA} / \mathrm{Lpq})+12 *(\mathrm{Lqq} / \mathrm{Lpq}) \\
& \mathrm{S} 113<-(\mathrm{LA} / \mathrm{Lpq})+13 *(\mathrm{Lqq} / \mathrm{Lpq}) \\
& \mathrm{S} 114<-(\mathrm{LA} / \mathrm{Lpq})+14 *(\mathrm{Lqq} / \mathrm{Lpq}) \\
& \mathrm{S} 115<-(\mathrm{LA} / \mathrm{Lpq})+15 *(\mathrm{Lqq} / \mathrm{Lpq}) \\
& \mathrm{S} 116<-(\mathrm{LA} / \mathrm{Lpq})+16 *(\mathrm{Lqq} / \mathrm{Lpq}) \\
& \mathrm{S} 117<-(\mathrm{LA} / \mathrm{Lpq})+17 *(\mathrm{Lqq} / \mathrm{Lpq}) \\
& \mathrm{S} 118<-(\mathrm{LA} / \mathrm{Lpq})+18 *(\mathrm{Lqq} / \mathrm{Lpq}) \\
& \mathrm{S} 119<-(\mathrm{LA} / \mathrm{Lpq})+19 *(\mathrm{Lqq} / \mathrm{Lpq}) \\
& \mathrm{S} 120<-(\mathrm{LA} / \mathrm{Lpq})+20 *(\mathrm{Lqq} / \mathrm{Lpq}) \\
& \mathrm{S} 121<-(\mathrm{LA} / \mathrm{Lpq})+21 *(\mathrm{Lqq} / \mathrm{Lpq}) \\
& \mathrm{S} 122<-(\mathrm{LA} / \mathrm{Lpq})+22 *(\mathrm{Lqq} / \mathrm{Lpq}) \\
& \mathrm{S} 123<-(\mathrm{LA} / \mathrm{Lpq})+23 *(\mathrm{Lqq} / \mathrm{Lpq}) \\
& \mathrm{S} 124<-(\mathrm{LA} / \mathrm{Lpq})+24 *(\mathrm{Lqq} / \mathrm{Lpq}) \\
& \mathrm{S} 125<-(\mathrm{LA} / \mathrm{Lpq})+25 *(\mathrm{Lqq} / \mathrm{Lpq}) \\
& \mathrm{S} 126<-(\mathrm{LA} / \mathrm{Lpq})+26 *(\mathrm{Lqq} / \mathrm{Lpq}) \\
& \mathrm{S} 127<-(\mathrm{LA} / \mathrm{Lpq})+27 *(\mathrm{Lqq} / \mathrm{Lpq}) \\
& \mathrm{S} 128<-(\mathrm{LA} / \mathrm{Lpq})+28 *(\mathrm{Lqq} / \mathrm{Lpq}) \\
& \mathrm{S} 129<-(\mathrm{LA} / \mathrm{Lpq})+29 *(\mathrm{Lqq} / \mathrm{Lpq}) \\
& \mathrm{S} 130<-(\mathrm{LA} / \mathrm{Lpq})+30 *(\mathrm{Lqq} / \mathrm{Lpq}) \\
& \mathrm{S} 131<-(\mathrm{LA} / \mathrm{Lpq})+31 *(\mathrm{Lqq} / \mathrm{Lpq}) \\
& \mathrm{S} 132<-(\mathrm{LA} / \mathrm{Lpq})+32 *(\mathrm{Lqq} / \mathrm{Lpq}) \\
& \mathrm{S} 133<-(\mathrm{LA} / \mathrm{Lpq})+33 *(\mathrm{Lqq} / \mathrm{Lpq})
\end{aligned}
$$




$$
\begin{aligned}
& \mathrm{S} 134<-(\mathrm{LA} / \mathrm{Lpq})+34 *(\mathrm{Lqq} / \mathrm{Lpq}) \\
& \mathrm{S} 135<-(\mathrm{LA} / \mathrm{Lpq})+35 *(\mathrm{Lqq} / \mathrm{Lpq}) \\
& \mathrm{S} 136<-(\mathrm{LA} / \mathrm{Lpq})+36 *(\mathrm{Lqq} / \mathrm{Lpq}) \\
& \mathrm{S} 137<-(\mathrm{LA} / \mathrm{Lpq})+37 *(\mathrm{Lqq} / \mathrm{Lpq}) \\
& \mathrm{S} 138<-(\mathrm{LA} / \mathrm{Lpq})+38 *(\mathrm{Lqq} / \mathrm{Lpq}) \\
& \mathrm{S} 139<-(\mathrm{LA} / \mathrm{Lpq})+39 *(\mathrm{Lqq} / \mathrm{Lpq}) \\
& \mathrm{S} 140<-(\mathrm{LA} / \mathrm{Lpq})+40 *(\mathrm{Lqq} / \mathrm{Lpq}) \\
& \mathrm{S} 141<-(\mathrm{LA} / \mathrm{Lpq})+41 *(\mathrm{Lqq} / \mathrm{Lpq}) \\
& \mathrm{S} 142<-(\mathrm{LA} / \mathrm{Lpq})+42 *(\mathrm{Lqq} / \mathrm{Lpq}) \\
& \mathrm{S} 143<-(\mathrm{LA} / \mathrm{Lpq})+43 *(\mathrm{Lqq} / \mathrm{Lpq}) \\
& \mathrm{S} 144<-(\mathrm{LA} / \mathrm{Lpq})+44 *(\mathrm{Lqq} / \mathrm{Lpq}) \\
& \mathrm{S} 145<-(\mathrm{LA} / \mathrm{Lpq})+45 *(\mathrm{Lqq} / \mathrm{Lpq}) \\
& \mathrm{S} 146<-(\mathrm{LA} / \mathrm{Lpq})+46 *(\mathrm{Lqq} / \mathrm{Lpq}) \\
& \mathrm{S} 147<-(\mathrm{LA} / \mathrm{Lpq})+47 *(\mathrm{Lqq} / \mathrm{Lpq}) \\
& \mathrm{S} 148<-(\mathrm{LA} / \mathrm{Lpq})+48 *(\mathrm{Lqq} / \mathrm{Lpq}) \\
& \mathrm{S} 149<-(\mathrm{LA} / \mathrm{Lpq})+49 *(\mathrm{Lqq} / \mathrm{Lpq}) \\
& \mathrm{S} 150<-(\mathrm{LA} / \mathrm{Lpq})+50 *(\mathrm{Lqq} / \mathrm{Lpq}) \\
& \mathrm{S} 151<-(\mathrm{LA} / \mathrm{Lpq})+51 *(\mathrm{Lqq} / \mathrm{Lpq}) \\
& \mathrm{S} 152<-(\mathrm{LA} / \mathrm{Lpq})+52 *(\mathrm{Lqq} / \mathrm{Lpq}) \\
& \mathrm{S} 153<-(\mathrm{LA} / \mathrm{Lpq})+53 *(\mathrm{Lqq} / \mathrm{Lpq}) \\
& \mathrm{S} 154<-(\mathrm{LA} / \mathrm{Lpq})+54 *(\mathrm{Lqq} / \mathrm{Lpq}) \\
& \mathrm{S} 155<-(\mathrm{LA} / \mathrm{Lpq})+55 *(\mathrm{Lqq} / \mathrm{Lpq}) \\
& \mathrm{S} 156<-(\mathrm{LA} / \mathrm{Lpq})+56 *(\mathrm{Lqq} / \mathrm{Lpq}) \\
& \mathrm{S} 157<-(\mathrm{LA} / \mathrm{Lpq})+57 *(\mathrm{Lqq} / \mathrm{Lpq}) \\
& \mathrm{S} 158<-(\mathrm{LA} / \mathrm{Lpq})+58 *(\mathrm{Lqq} / \mathrm{Lpq}) \\
& \mathrm{S} 159<-(\mathrm{LA} / \mathrm{Lpq})+59 *(\mathrm{Lqq} / \mathrm{Lpq}) \\
& \mathrm{S} 160<-(\mathrm{LA} / \mathrm{Lpq})+60 *(\mathrm{Lqq} / \mathrm{Lpq}) \\
& \mathrm{S} 161<-(\mathrm{LA} / \mathrm{Lpq})+61 *(\mathrm{Lqq} / \mathrm{Lpq}) \\
& \mathrm{S} 162<-(\mathrm{LA} / \mathrm{Lpq})+62 *(\mathrm{Lqq} / \mathrm{Lpq}) \\
& \mathrm{S} 163<-(\mathrm{LA} / \mathrm{Lpq})+63 *(\mathrm{Lqq} / \mathrm{Lpq}) \\
& \mathrm{S} 164<-(\mathrm{LA} / \mathrm{Lpq})+64 *(\mathrm{Lqq} / \mathrm{Lpq}) \\
& \mathrm{S} 165<-(\mathrm{LA} / \mathrm{Lpq})+65 *(\mathrm{Lqq} / \mathrm{Lpq})
\end{aligned}
$$




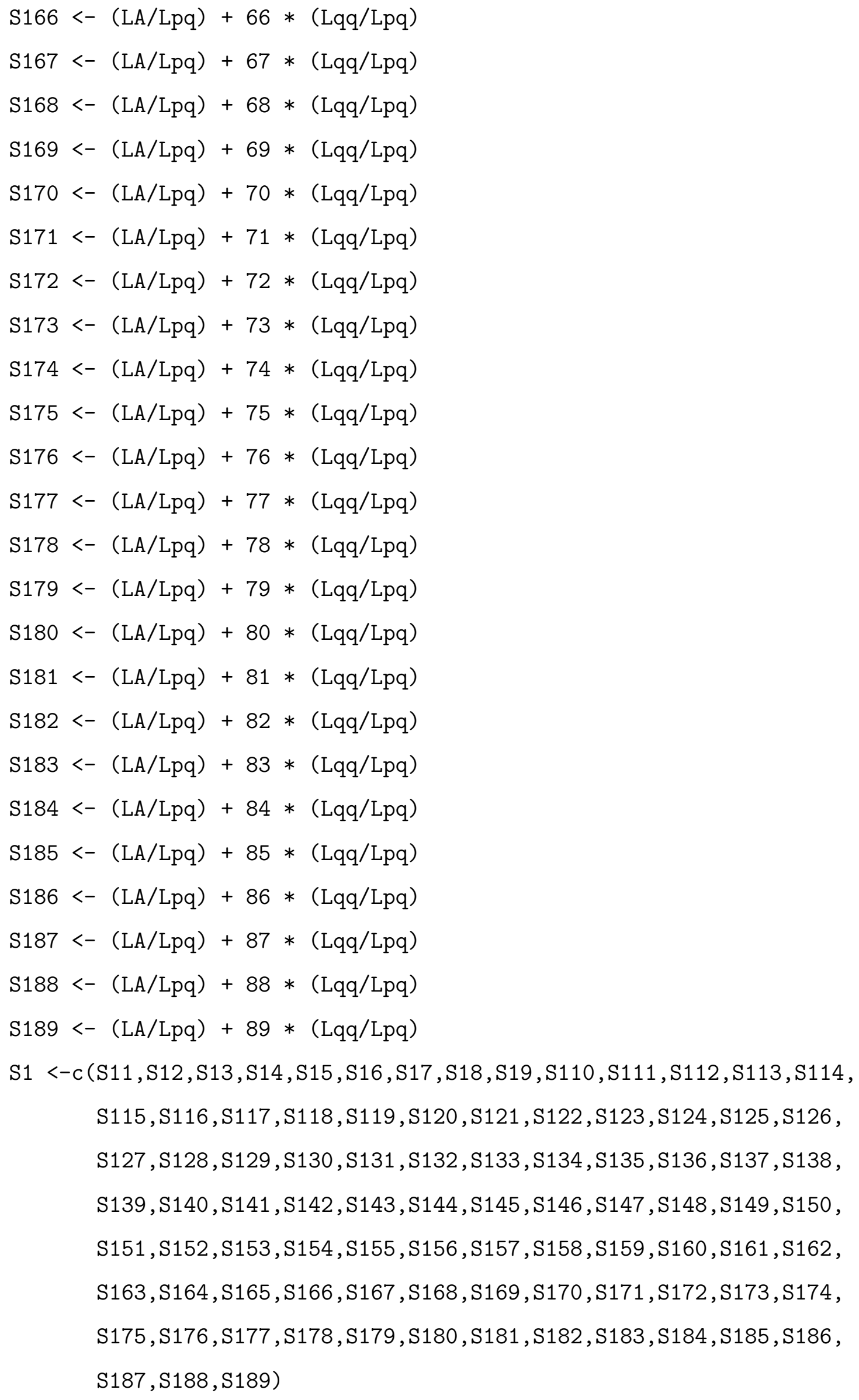


qq

Lqq <- $\log (q q)$

Lqq

$p q<-((p 1 * q 0) /(p 0 * q 1))$

$\mathrm{pq}$

$L p q<-\log (p q)$

Lpq

$\mathrm{m}<-c(1,2,3,4,5,6,7,8,9,10,11,12,13,14,15,16,17$, $18,19,20,21,22,23,24,25,26,27,28,29,30,31,32$, $33,34,35,36,37,38,39,40)$

$\mathrm{m}$

$\mathrm{km}<-c(0.4,0.8,1.2,1.6,2.0,2.4,2.8,3.2,3.6,4.0,4.4,4.8,5.2,5.6,6.0$, $6.4,6.8,7.2,7.6,8.0,8.4,8.8,9.2,9.6,10.0,10.4,10.8,11.2,11.6$, $12.0,12.4,12.8,13.2,13.6,14.0,14.4,14.8,15.2,15.6,16.0)$

$\mathrm{km}$

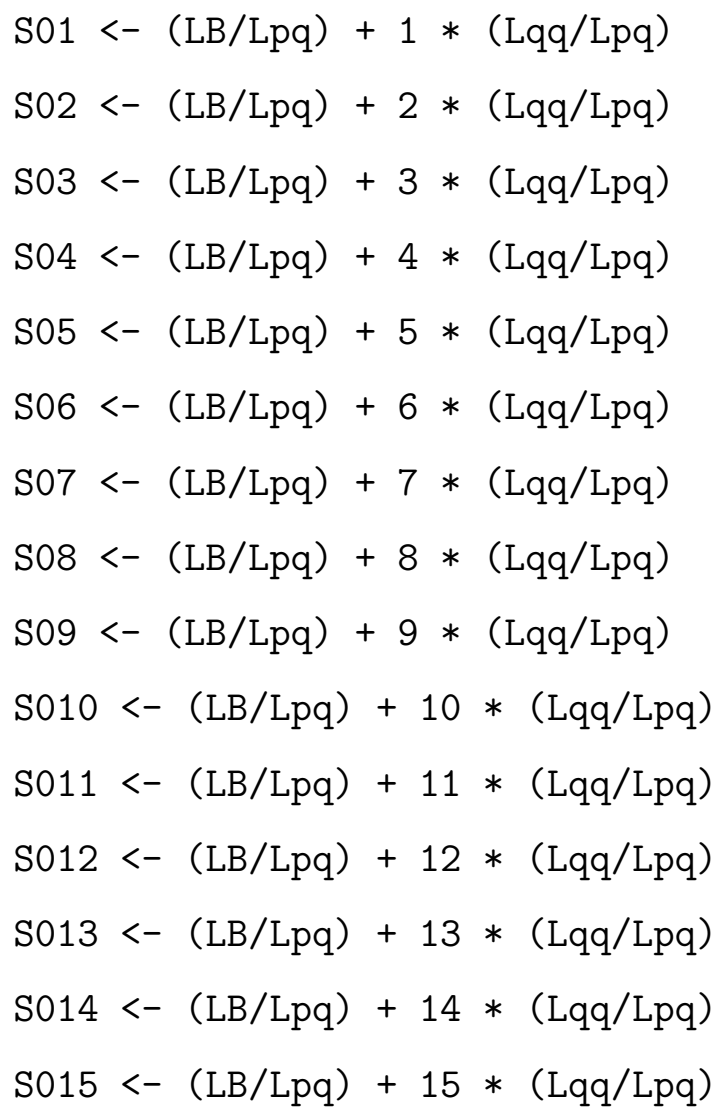




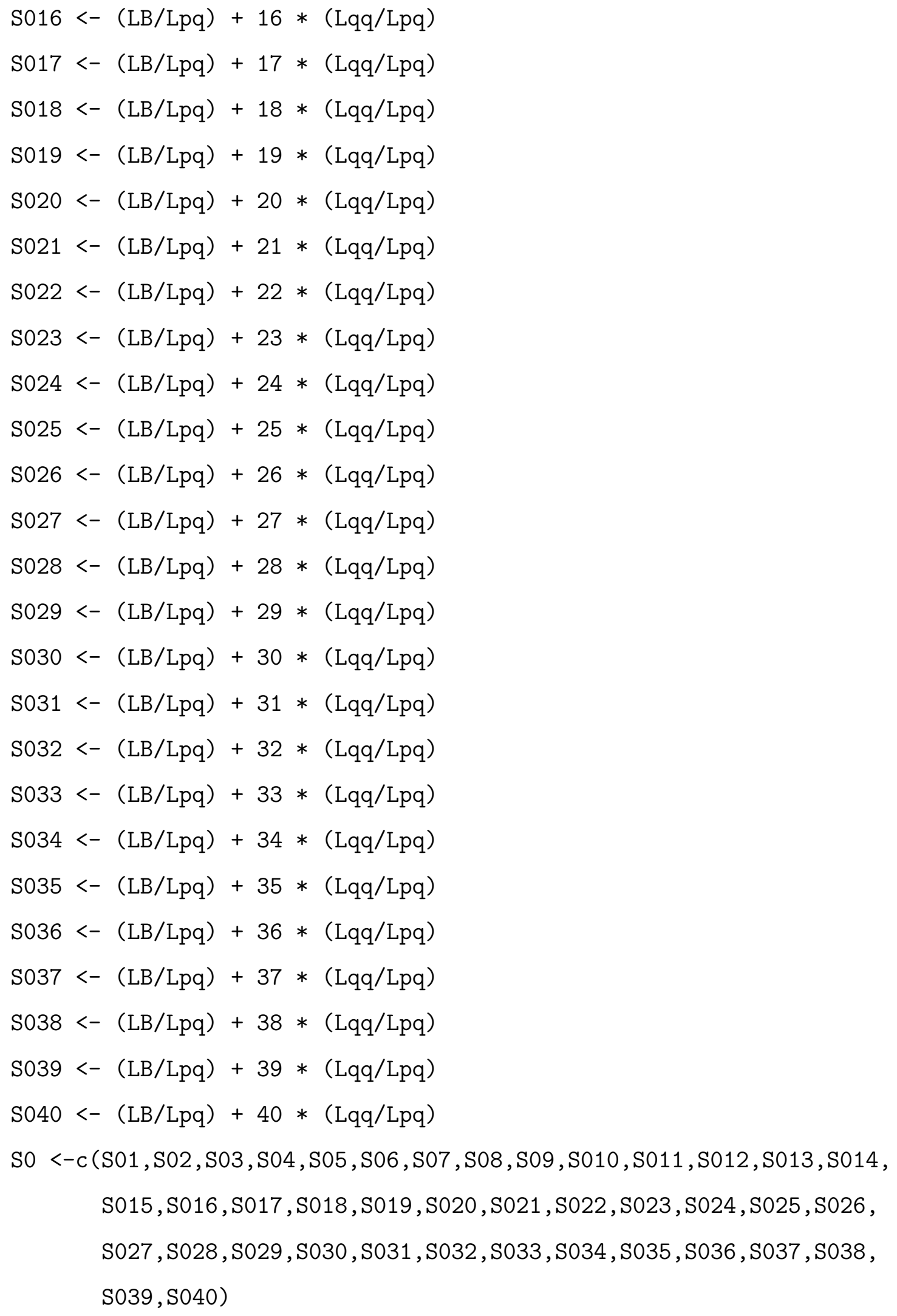




$$
\begin{aligned}
& \mathrm{S} 12<-(\mathrm{LA} / \mathrm{Lpq})+2 *(\mathrm{Lqq} / \mathrm{Lpq}) \\
& \mathrm{S} 13<-(\mathrm{LA} / \mathrm{Lpq})+3 *(\mathrm{Lqq} / \mathrm{Lpq}) \\
& \mathrm{S} 14<-(\mathrm{LA} / \mathrm{Lpq})+4 *(\mathrm{Lqq} / \mathrm{Lpq}) \\
& \mathrm{S} 15<-(\mathrm{LA} / \mathrm{Lpq})+5 *(\mathrm{Lqq} / \mathrm{Lpq}) \\
& \mathrm{S} 16<-(\mathrm{LA} / \mathrm{Lpq})+6 *(\mathrm{Lqq} / \mathrm{Lpq}) \\
& \mathrm{S} 17<-(\mathrm{LA} / \mathrm{Lpq})+7 *(\mathrm{Lqq} / \mathrm{Lpq}) \\
& \mathrm{S} 18<-(\mathrm{LA} / \mathrm{Lpq})+8 *(\mathrm{Lqq} / \mathrm{Lpq}) \\
& \mathrm{S} 19<-(\mathrm{LA} / \mathrm{Lpq})+9 *(\mathrm{Lqq} / \mathrm{Lpq}) \\
& \mathrm{S} 110<-(\mathrm{LA} / \mathrm{Lpq})+10 *(\mathrm{Lqq} / \mathrm{Lpq}) \\
& \mathrm{S} 111<-(\mathrm{LA} / \mathrm{Lpq})+11 *(\mathrm{Lqq} / \mathrm{Lpq}) \\
& \mathrm{S} 112<-(\mathrm{LA} / \mathrm{Lpq})+12 *(\mathrm{Lqq} / \mathrm{Lpq}) \\
& \mathrm{S} 113<-(\mathrm{LA} / \mathrm{Lpq})+13 *(\mathrm{Lqq} / \mathrm{Lpq}) \\
& \mathrm{S} 114<-(\mathrm{LA} / \mathrm{Lpq})+14 *(\mathrm{Lqq} / \mathrm{Lpq}) \\
& \mathrm{S} 115<-(\mathrm{LA} / \mathrm{Lpq})+15 *(\mathrm{Lqq} / \mathrm{Lpq}) \\
& \mathrm{S} 116<-(\mathrm{LA} / \mathrm{Lpq})+16 *(\mathrm{Lqq} / \mathrm{Lpq}) \\
& \mathrm{S} 117<-(\mathrm{LA} / \mathrm{Lpq})+17 *(\mathrm{Lqq} / \mathrm{Lpq}) \\
& \mathrm{S} 118<-(\mathrm{LA} / \mathrm{Lpq})+18 *(\mathrm{Lqq} / \mathrm{Lpq}) \\
& \mathrm{S} 119<-(\mathrm{LA} / \mathrm{Lpq})+19 *(\mathrm{Lqq} / \mathrm{Lpq}) \\
& \mathrm{S} 120<-(\mathrm{LA} / \mathrm{Lpq})+20 *(\mathrm{Lqq} / \mathrm{Lpq}) \\
& \mathrm{S} 121<-(\mathrm{LA} / \mathrm{Lpq})+21 *(\mathrm{Lqq} / \mathrm{Lpq}) \\
& \mathrm{S} 122<-(\mathrm{LA} / \mathrm{Lpq})+22 *(\mathrm{Lqq} / \mathrm{Lpq}) \\
& \mathrm{S} 123<-(\mathrm{LA} / \mathrm{Lpq})+23 *(\mathrm{Lqq} / \mathrm{Lpq}) \\
& \mathrm{S} 124<-(\mathrm{LA} / \mathrm{Lpq})+24 *(\mathrm{Lqq} / \mathrm{Lpq}) \\
& \mathrm{S} 125<-(\mathrm{LA} / \mathrm{Lpq})+25 *(\mathrm{Lqq} / \mathrm{Lpq}) \\
& \mathrm{S} 126<-(\mathrm{LA} / \mathrm{Lpq})+26 *(\mathrm{Lqq} / \mathrm{Lpq}) \\
& \mathrm{S} 127<-(\mathrm{LA} / \mathrm{Lpq})+27 *(\mathrm{Lqq} / \mathrm{Lpq}) \\
& \mathrm{S} 128<-(\mathrm{LA} / \mathrm{Lpq})+28 *(\mathrm{Lqq} / \mathrm{Lpq}) \\
& \mathrm{S} 129<-(\mathrm{LA} / \mathrm{Lpq})+29 *(\mathrm{Lqq} / \mathrm{Lpq}) \\
& \mathrm{S} 130<-(\mathrm{LA} / \mathrm{Lpq})+30 *(\mathrm{Lqq} / \mathrm{Lpq}) \\
& \mathrm{S} 131<-(\mathrm{LA} / \mathrm{Lpq})+31 *(\mathrm{Lqq} / \mathrm{Lpq}) \\
& \mathrm{S} 132<-(\mathrm{LA} / \mathrm{Lpq})+32 *(\mathrm{Lqq} / \mathrm{Lpq}) \\
& \mathrm{S} 133<-(\mathrm{LA} / \mathrm{Lpq})+33 *(\mathrm{Lqq} / \mathrm{Lpq})
\end{aligned}
$$




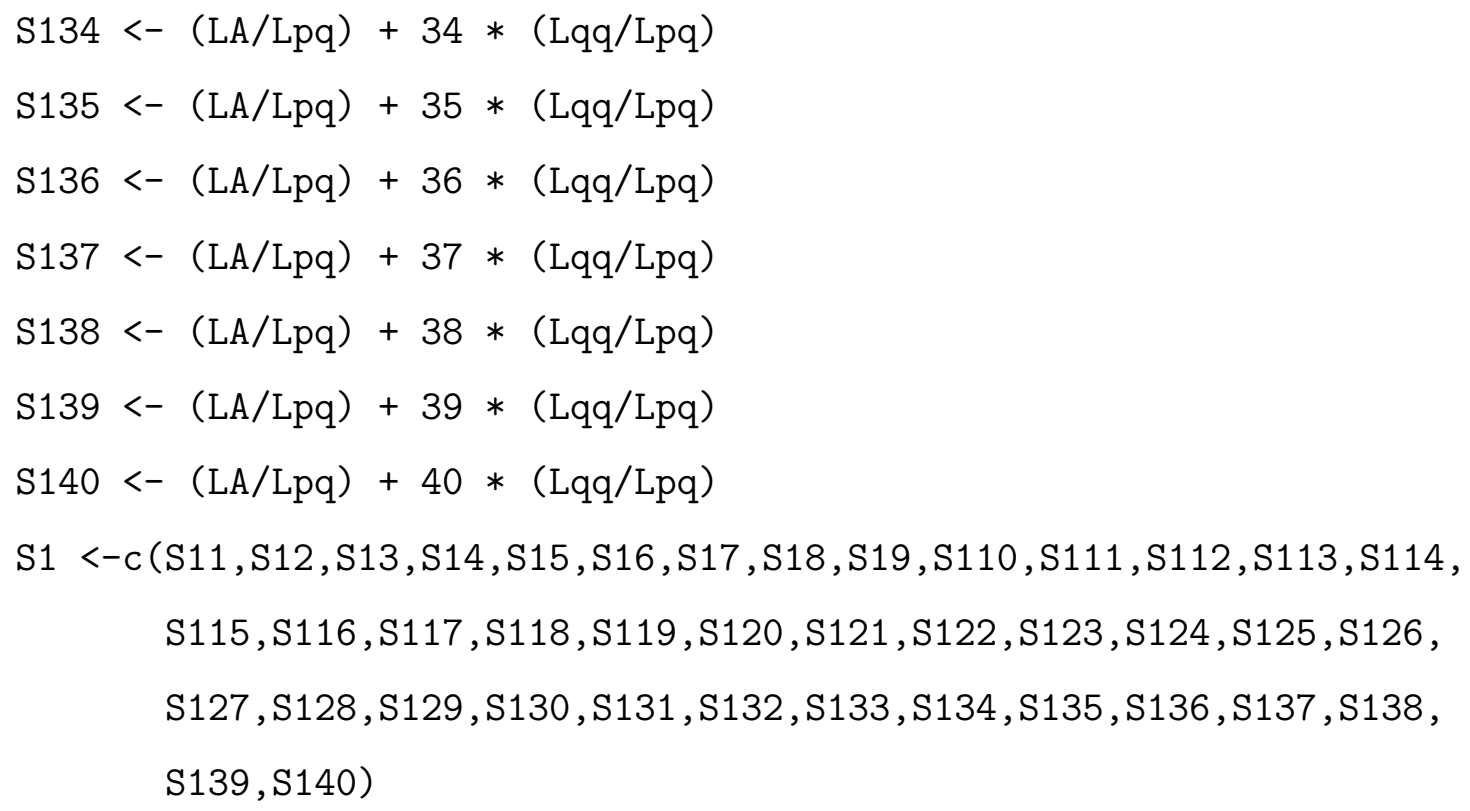


ANEXO D - Construção da Curva Característica Operacional e da Curva do Número Médio de Unidades Amostrais, com níveis de erros iguais a 0,10 e 0,20.

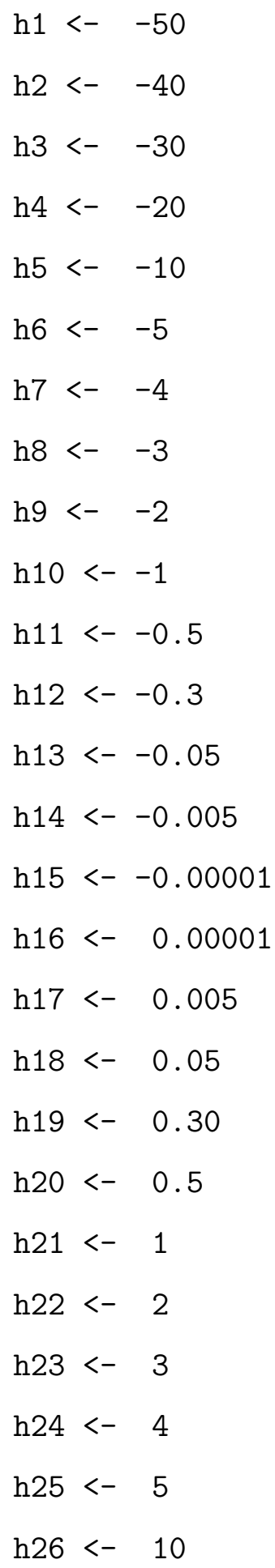




$$
\begin{aligned}
& \text { h27 <- } 20 \\
& \text { h28 <- } 30 \\
& \text { h29 - } 40 \\
& \text { h30<- } 50
\end{aligned}
$$

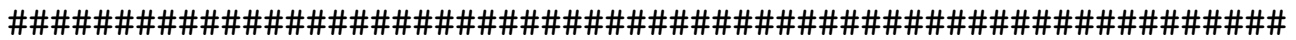

Valores de $\mathrm{p}$ obtidos em função dos valores da constante $\mathrm{h}$.

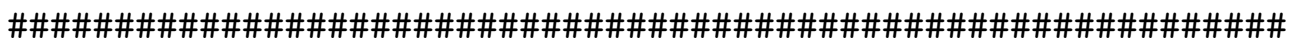

$$
\begin{aligned}
& \text { ph1 <- }\left(1-((1-\mathrm{p} 1) /(1-\mathrm{p} 0))^{\wedge} \mathrm{h} 1\right) /\left((\mathrm{p} 1 / \mathrm{p} 0)^{\wedge} \mathrm{h} 1-((1-\mathrm{p} 1) /(1-\mathrm{p} 0))^{\wedge} \mathrm{h} 1\right) \\
& \mathrm{ph} 2<-\left(1-((1-\mathrm{p} 1) /(1-\mathrm{p} 0))^{\wedge} \mathrm{h} 2\right) /\left((\mathrm{p} 1 / \mathrm{p} 0)^{\wedge} \mathrm{h} 2-((1-\mathrm{p} 1) /(1-\mathrm{p} 0))^{\wedge} \mathrm{h} 2\right)
\end{aligned}
$$

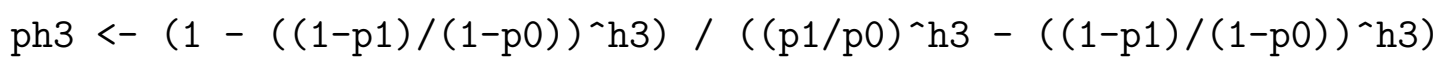

$$
\begin{aligned}
& \mathrm{ph} 4<-\left(1-((1-\mathrm{p} 1) /(1-\mathrm{p} 0))^{\wedge} \mathrm{h} 4\right) /\left((\mathrm{p} 1 / \mathrm{p} 0)^{\wedge} \mathrm{h} 4-((1-\mathrm{p} 1) /(1-\mathrm{p} 0))^{\wedge} \mathrm{h} 4\right) \\
& \mathrm{ph} 5<-\left(1-((1-\mathrm{p} 1) /(1-\mathrm{p} 0))^{\wedge} \mathrm{h} 5\right) /\left((\mathrm{p} 1 / \mathrm{p} 0)^{\wedge} \mathrm{h} 5-((1-\mathrm{p} 1) /(1-\mathrm{p} 0))^{\wedge} \mathrm{h} 5\right) \\
& \text { ph6 <- }\left(1-((1-\mathrm{p} 1) /(1-\mathrm{p} 0))^{\wedge} \mathrm{h} 6\right) /\left((\mathrm{p} 1 / \mathrm{p} 0)^{\wedge} \mathrm{h} 6-((1-\mathrm{p} 1) /(1-\mathrm{p} 0))^{\wedge} \mathrm{h} 6\right) \\
& \mathrm{ph} 7<-\left(1-((1-\mathrm{p} 1) /(1-\mathrm{p} 0))^{\wedge} \mathrm{h} 7\right) /\left((\mathrm{p} 1 / \mathrm{p} 0)^{\wedge} \mathrm{h} 7-((1-\mathrm{p} 1) /(1-\mathrm{p} 0))^{\wedge} \mathrm{h} 7\right) \\
& \text { ph8 <- (1-((1-p1)/(1-p0) )^h8) / ((p1/p0)^h8 - ((1-p1)/(1-p0))^h8) } \\
& \text { ph9 <- }\left(1-((1-\mathrm{p} 1) /(1-\mathrm{p} 0))^{\wedge} \mathrm{h} 9\right) /\left((\mathrm{p} 1 / \mathrm{p} 0)^{\wedge} \mathrm{h} 9-((1-\mathrm{p} 1) /(1-\mathrm{p} 0))^{\wedge} \mathrm{h} 9\right) \\
& \text { ph10<- }(1-((1-\mathrm{p} 1) /(1-\mathrm{p} 0)) \wedge \mathrm{h} 10) /\left((\mathrm{p} 1 / \mathrm{p} 0)^{\wedge} \mathrm{h} 10-((1-\mathrm{p} 1) /(1-\mathrm{p} 0))^{\wedge} \mathrm{h} 10\right)
\end{aligned}
$$

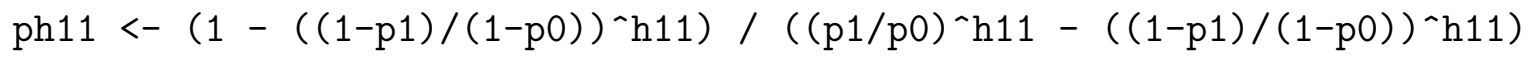

$$
\begin{aligned}
& \text { ph12 <- }\left(1-((1-p 1) /(1-p 0))^{\wedge h} 12\right) /\left((p 1 / p 0)^{\wedge} h 12-((1-p 1) /(1-p 0))^{\wedge} h 12\right)
\end{aligned}
$$

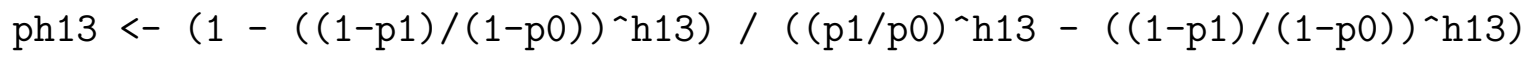

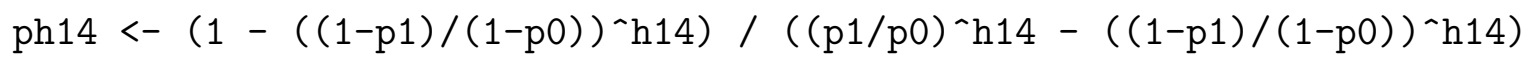

$$
\begin{aligned}
& \text { ph15 <- }\left(1-((1-\mathrm{p} 1) /(1-\mathrm{p} 0))^{\wedge} \mathrm{h} 15\right) /\left((\mathrm{p} 1 / \mathrm{p} 0)^{\wedge} \mathrm{h} 15-((1-\mathrm{p} 1) /(1-\mathrm{p} 0))^{\wedge} \mathrm{h} 15\right)
\end{aligned}
$$

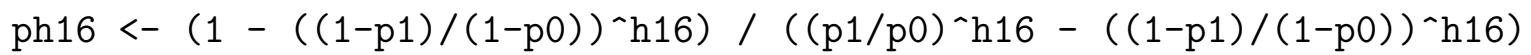

$$
\begin{aligned}
& \text { ph17 <- }(1-((1-\mathrm{p} 1) /(1-\mathrm{p} 0)) \wedge h 17) /\left((\mathrm{p} 1 / \mathrm{p} 0)^{\wedge} \mathrm{h} 17-((1-\mathrm{p} 1) /(1-\mathrm{p} 0))^{\wedge} \mathrm{h} 17\right)
\end{aligned}
$$

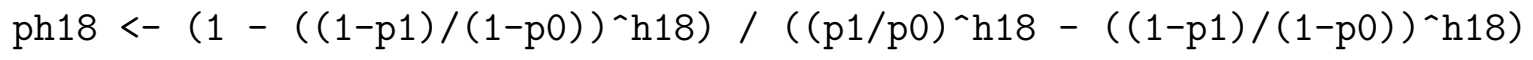

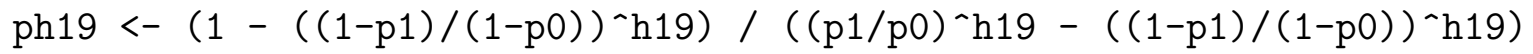

$$
\begin{aligned}
& \text { ph20<- }\left(1-((1-\mathrm{p} 1) /(1-\mathrm{p} 0))^{\wedge} \mathrm{h} 20\right) /\left((\mathrm{p} 1 / \mathrm{p} 0)^{\wedge} \mathrm{h} 20-((1-\mathrm{p} 1) /(1-\mathrm{p} 0))^{\wedge} \mathrm{h} 20\right) \\
& \mathrm{ph} 21<-(1-((1-\mathrm{p} 1) /(1-\mathrm{p} 0)) \wedge \mathrm{h} 21) /\left((\mathrm{p} 1 / \mathrm{p} 0)^{\wedge} \mathrm{h} 21-((1-\mathrm{p} 1) /(1-\mathrm{p} 0))^{\wedge} \mathrm{h} 21\right) \\
& \text { ph2 <- }\left(1-((1-\mathrm{p} 1) /(1-\mathrm{p} 0))^{\wedge} \mathrm{h} 22\right) /\left((\mathrm{p} 1 / \mathrm{p} 0)^{\wedge} \mathrm{h} 22-((1-\mathrm{p} 1) /(1-\mathrm{p} 0))^{\wedge} \mathrm{h} 22\right) \\
& \mathrm{ph} 23<-\left(1-((1-\mathrm{p} 1) /(1-\mathrm{p} 0))^{\wedge} \mathrm{h} 23\right) /\left((\mathrm{p} 1 / \mathrm{p} 0)^{\wedge} \mathrm{h} 23-((1-\mathrm{p} 1) /(1-\mathrm{p} 0))^{\wedge} \mathrm{h} 23\right)
\end{aligned}
$$




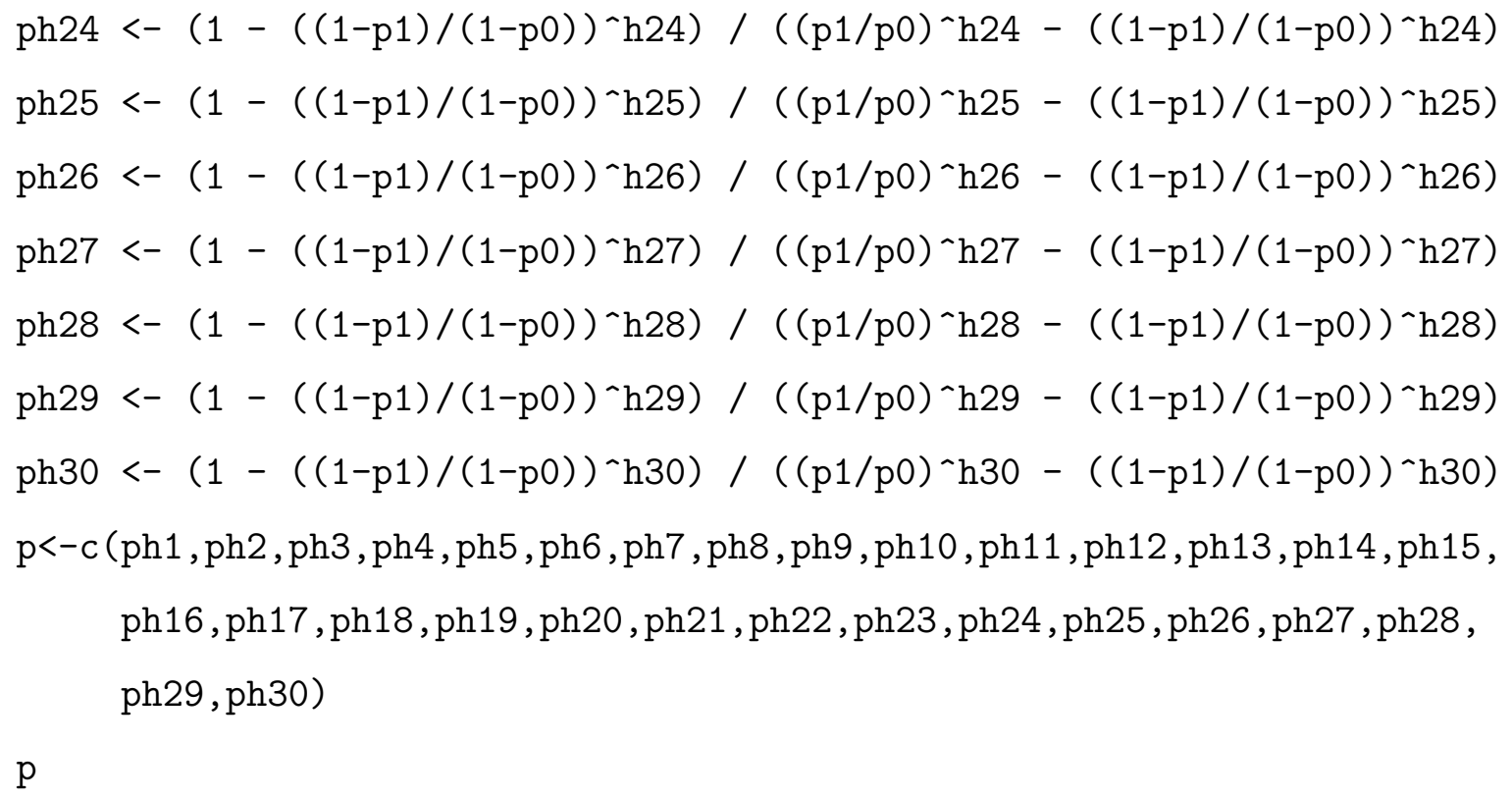

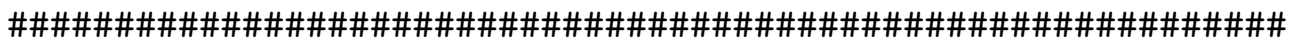

Pontos da Curva Característica Operacional determinados em função dos valores de $h$.

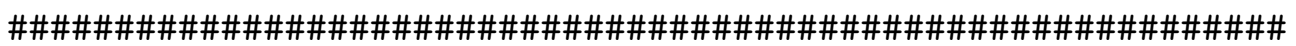

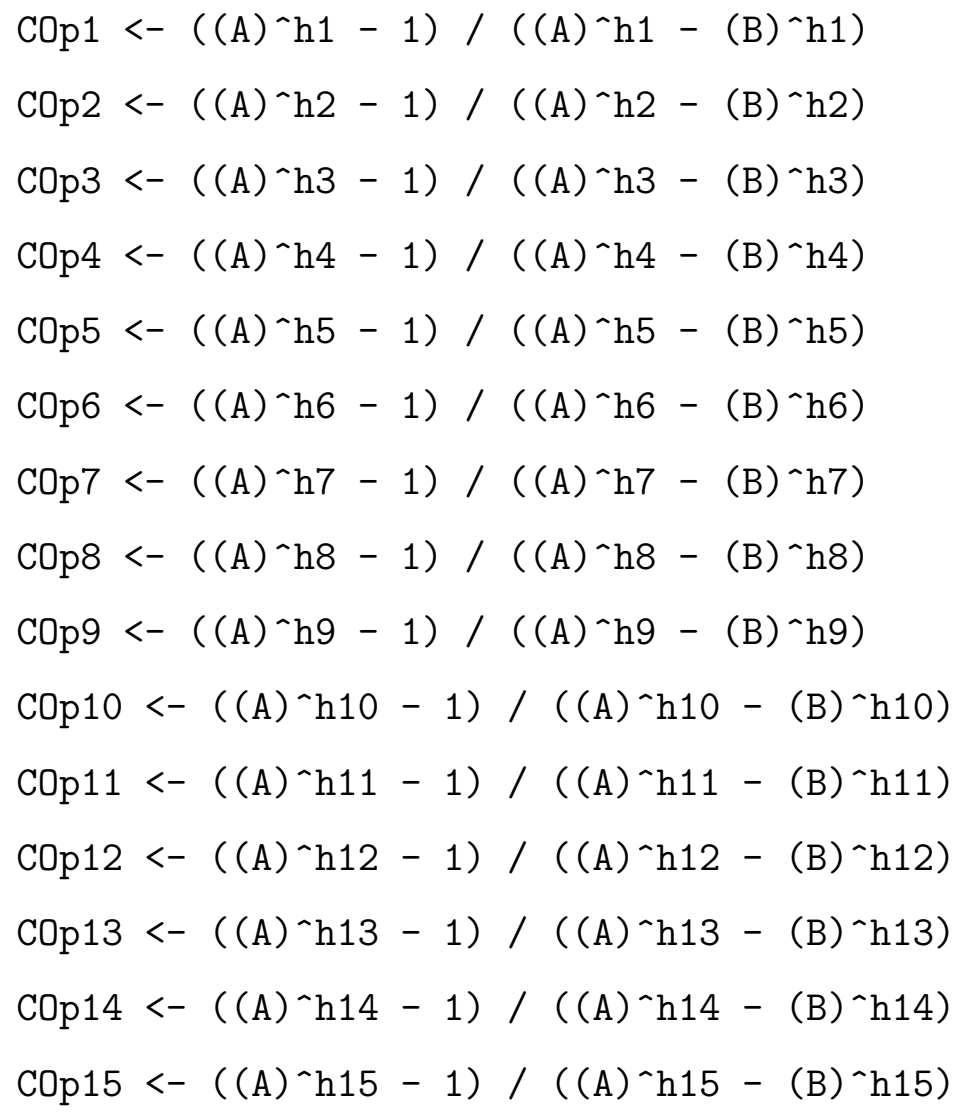




$$
\begin{aligned}
& \text { COp16 <- ((A)^h16 - 1) / ((A)^h16 - (B)^h16) } \\
& \text { COp17 <- }\left((\mathrm{A})^{\wedge} \mathrm{h} 17-1\right) /\left((\mathrm{A})^{\wedge} \mathrm{h} 17-(\mathrm{B})^{\wedge} \mathrm{h} 17\right) \\
& \text { COp18 <- ((A)^h18 - 1) / ((A)^h18 - (B)^h18) } \\
& \text { COp19 <- ((A)^h19 - 1) / ((A)^h19 - (B)^h19) } \\
& \text { COp20 <- ((A)^h20 - 1) / ((A)^h20 - (B)^h20) } \\
& \text { COp21 <- ((A)^h21 - 1) / ((A)^h21 - (B)^h21) } \\
& \text { COp2 }<-\left((A)^{\wedge} h 22-1\right) /\left((A)^{\wedge} h 22-(B)^{\wedge} h 22\right) \\
& \mathrm{COp} 23<-\left((\mathrm{A})^{\wedge} \mathrm{h} 23-1\right) /\left((\mathrm{A})^{\wedge} \mathrm{h} 23-(\mathrm{B})^{\wedge} \mathrm{h} 23\right) \\
& \text { COp24 <- ((A)^h24 - 1) / ((A)^h24 - (B)^h24) } \\
& \text { COp25 <- ((A)^h25 - 1) / ((A)^h25 - (B)^h25) } \\
& \text { COp26 <- ((A)^h26 - 1) / ((A)^h26 - (B)^h26) } \\
& \text { COp27 <- ((A)^h27 - 1) / ((A)^h27 - (B)^h27) } \\
& \text { COp28<- ((A)^h28 - 1) / ((A)^h28 - (B)^h28) } \\
& \text { COp29 <- ((A)^h29 - 1) / ((A)^h29 - (B)^h29) } \\
& \text { COp30<-((A)^h30 - 1) / ((A)^h30 - (B)^h30) }
\end{aligned}
$$

$\mathrm{COp}<-\mathrm{c}(\mathrm{COp} 1, \mathrm{COp} 2, \mathrm{COp} 3, \mathrm{COp} 4, \mathrm{COp} 5, \mathrm{COp} 6, \mathrm{COp} 7, \mathrm{COp} 8, \mathrm{COp} 9, \mathrm{COp} 10, \mathrm{COp} 11$, COp12, COp13, COp14, COp15, C0p16, C0p17, COp18, C0p19, C0p20 , C0p21 , COp22, COp23, COp24, COp25, COp26, COp27, COp28, COp29, COp30)

$\mathrm{COp}$

$\operatorname{plot}(\mathrm{p}, \mathrm{COp}$, type $=" \mathrm{n} ")$

$\operatorname{lines}(\mathrm{p}, \mathrm{CO})$

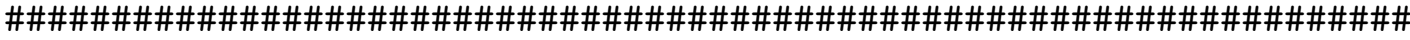 Pontos da Curva do Número Esperado de Unidades Amostrais obtidos em função dos valores de $\mathrm{p}$ e COp.}

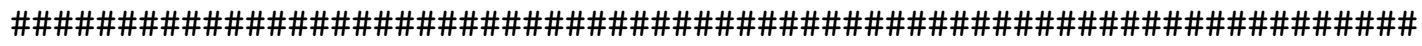

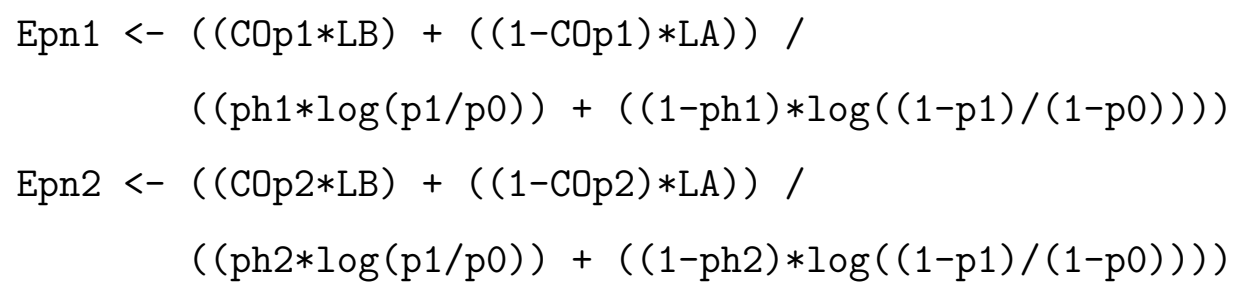




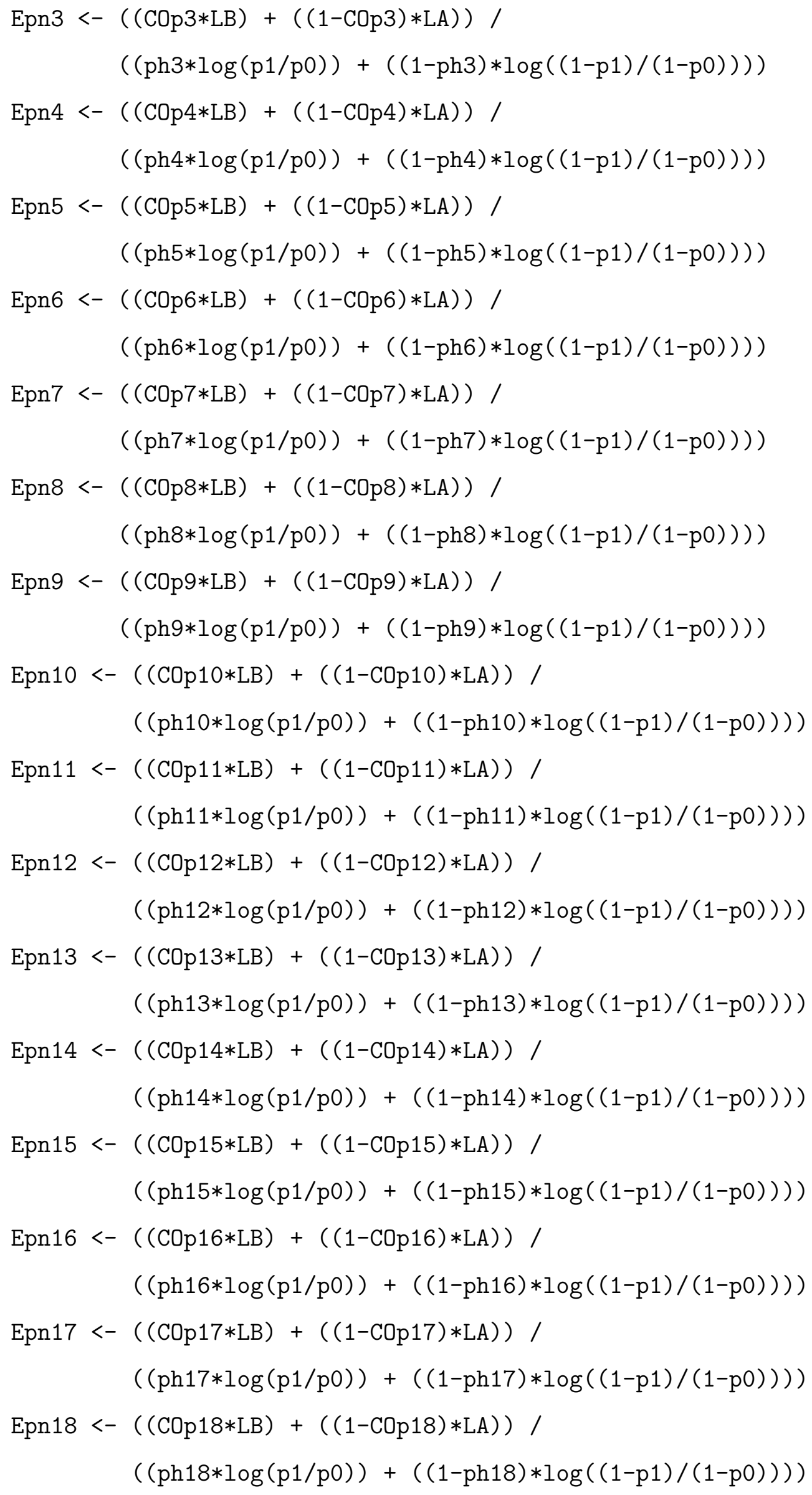




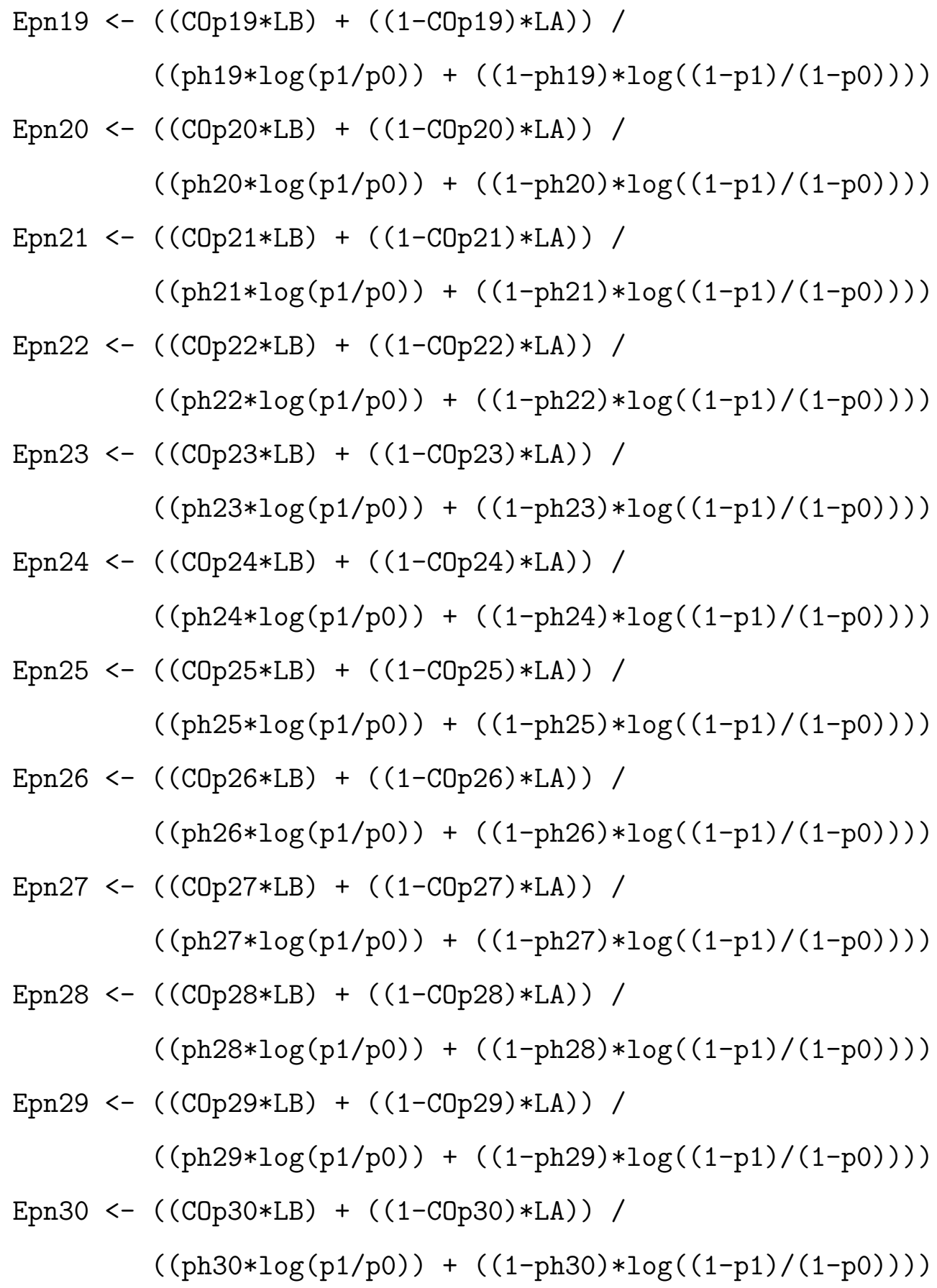




\section{REFERÊNCIAS BIBLIOGRÁFICAS}

ANSCOMBE, F. J. The statistical analysis of insect counts based on the negative binomial distribution. Biometrics, v.5, p.165-173, 1949.

BARBOSA, J. C. A amostragem seqüencial. In: FERNANDES, O. A.; CORREIA, A. C. B.; DE BORTOLI, S.A. (Eds.), Manejo integrado de pragas e nematóides. Jaboticabal, FUNEP, 1992. p.205-211.

BARBOSA, J. C. Distribuições de probabilidade como base para análises estatísticas, amostragem e estratégias de manejo de Diatraea saccharalis (Fabr., 1794) na cultura da canade-açúcar. Piracicaba, 1985. 131p. Tese (Doutorado) - Escola Superior de Agricultura "Luiz de Queiroz", Universidade de São Paulo.

BEARZOTI, E.; AQUINO, L. H. Plano de amostragem seqüencial para avaliação de infestação de bicho-mineiro (Lepidoptera: Lyonetidae) no sul de Minas Gerais. Pesquisa Agropecuária Brasileira, v.29, n 5, p.695-705, 1994.

BECHINSKI, E. J.; STOLTZ, R. I. Presence-abscence sequential decision plans for Tetranychus urticae (Acari: Tetranychidae) in garden-seed beans, Phaseolus vulgaris. Journal of Economic Entomology, v.78, p.1475-1480, 1985.

BIANCO, R. Construção e validação de planos de amostragem para o manejo da lagarta do cartucho spodoptera frugiperda (J.E. Smith, 1797) (Lepidoptera: Noctuidae) na cultura do milho. Piracicaba, 1995, 103p. Tese (Doutorado) - Escola Superior de Agricultura "Luiz de Queiroz", Universidade de São Paulo.

BOSCH, R. V.; MESSENGER, P. S.; GUTIERREZ, A. P. An introduction to biological control. New York: Plenum Press, 1985. 247p.

CROCOMO, W.B. O que é o manejo de pragas. In: CROCOMO, W.B. (Org.). Manejo Integrado de Pragas. Botucatu: UNESP; São Paulo: CETESB, 1990. p.9-34. 
DANTAS, I. M. Distribuição espacial e plano de amostragem seqüencial para a lagarta do minador-dos-citros Phyllocnistis citrella STAINTON, 1856 (Lepdoptera: Gracillariidae), em laranjeira 'Pêra-Rio' Citrus sinensis (L.) OSBECK. Jaboticabal, 2002. 63p. Tese (Doutorado), Faculdade de Ciências Agrárias e Veterinárias, Universidade Estadual Paulista "Júlio de Mesquita Filho".

DA SILVA, J.; E SILVA, P. S. L.; NUNES, R. P. Amostragem e tamanho da amostra na estimação de caracteres da espiga do milho. Pesquisa Agropecuária Brasileira, v.33, n.12, p.1983-1988, 1998.

ESTEFANEL, V. A amostragem seqüencial baseada no teste seqüencial da razão de probabilidades e seu uso no controle das lagartas da soja no Estado do Rio Grande do Sul. Piracicaba, 1977.117p. Dissertação (Mestrado) - Escola Superior de Agricultura "Luiz de Queiroz", Universidade de São Paulo.

EVANS, D. A. Experimental evidence concerning contagious distributions in ecology. Biometrika, v.40, p.186-211, 1953.

FARIAS, P. R. S.; BARBOSA, J. C.; BUSOLI, A. C. Amostragem seqüencial com base na Lei de Taylor para levantamento de Spodoptera frugiperda na cultura do milho. Scientia Agricola, v.58, n.2, p.395-399, 2001a.

FARIAS, P. R. S.; BARBOSA, J. C.; BUSOLI, A. C. Amostragem seqüencial (presençaausência) para Spodoptera frugiperda (J.E. Smith) (Lepidoptera: Noctuidae) na cultura do milho. Neotropical Entomology, v.30, n.4, p.691-695, 2001b.

FARIAS, P. R. S.; BARBOSA, J. C.; BUSOLI, A. C. Distribuição espacial da lagarta do cartucho Spodoptera frugiperda (J.E. Smith) (Lepidoptera: Noctuidae) na cultura do milho. Neotropical Entomology, v.30, n.4, p.681-689, 2001c.

FERNANDES, M. G. Distribuição espacial e amostragem seqüencial dos principais noctuídeos do algodoeiro (Gossypium hirsutum L.). Jaboticabal, 2002. 140p. Tese (Doutorado) - Faculdade de Ciências Agrárias e Veterinárias, Universidade Estadual Paulista "Júlio de Mesquita Filho". 
FERNANDES, M. G.; BUSOLI, A. C.; BARBOSA, J. C. Amostragem seqüencial de Alabama argillacea (Hübner) (Lepidoptera: Noctuidae) em algodoeiro. Neotropical Entomology, v.32, n.1, p.117-122, 2003.

GOMIDE, E. V. A.; VILELA, E. F.; PICANÇO, M. Comparação de procedimentos de amostragem de Tuta absoluta (Meyrick) (Lepidoptera: Gelechiidae) em tomateiro estaqueado. Neotropical Entomology, v.30, n.4, p.697-705, 2001.

GRAVENA, S. Manejo integrado de pragas dos citros.Laranja, n.5, p.323-361, 1984.

GRAVENA, S.; FERNANDES, O. A.; PAZINI, W. C. Amostragem seqüencial para os ácaros da falsa ferrugem Phyllocoptruta oleivora (Ashm.) e da leprose Brevipalphus phoenicis (Geisks) no manejo integrado de pragas dos citros. Laranja, n.9, p.147-160, 1988.

HOLLINGSWORTH, C. S.; GATSONIS, C. A. Sequential sampling plans for green peach aphid (Homoptera: Aphididae) on potato. Journal of Economic Entomology, v.16, p.1365-1369, 1990.

LUNA, J. M.; FLEISCHER, S. J.; ALLEN, W. A. Development and validation of sequential sampling plans for potato leafhopper (Homoptera: Cicadellidae) in alfalfa. Environmental Entomology, v.12, n.6, p.1690-1694, 1983.

LYNCH, A. M.; FOWLER, G. W.; SIMMONS, G. A. Sequential sampling plans for spruce budworm (Lepidoptera:Tortricidae) egg mass density using Monte Carlo simulation. Journal of Economic Entomology, v.83, n.4, p.1479-1484, 1990.

MAITEKI, G. A.; LAMB, R. J. Sequential decision plan for control of pea aphid, Acyrthosiphon pisum (Homoptera: Aphididae), on field peas in Manitoba. Journal of Economic Entomology, v.80, n.3, p.605-607, 1987.

MOURA, M. F. DE; PICANÇO, M. C.; SILVA, E. M. DA; GUEDES, R. N. C.; PEREIRA, J. L. Plano de amostragem do biótipo B de Bemisia tabaci na cultura do pepino. Pesquisa Agropecuária Brasileira, v.38, n.12, p.1357-1363, 2003.

NYROP, J. P.; SIMMONS, G. A. Erros incurred when using Iwao's sequential decision rule in insect sampling. Environmental Entomology, v.13, n.6, p.1459-1465, 1984. 
NYROP, J. P.; WRIGHT, R. L. Use of double sample plans in insect sampling with reference to the Colorado potato beetle, Leptinotarsa decemlineata (Coleoptera: Chrysomelidae). Environmental Entomology, v.14, n.5, p.644-649, 1985.

OVERhOlt, W. A.; KNUTSOn, A. E.; SMITH JR, J. W.; GILSTRAP, F. E. Distribution and sampling of southwestern corn borer(Lepidoptera: Pyralidae). Journal of Economic Entomology, v.83, n.4, p.1370-1375, 1990.

PENG, C.; BREWER, G. J. Sequential plans for estimating achene damage by the red sunflower seed weevil (Coleoptera: Curculionidae). The Canadian Entomologist, v.127, p.7-14, 1995.

PERECIN, D.; BARBOSA, J. C. Amostragem e análise estatística de dados de distribuição de contágio. Revista de Matemática e Estatística, v.10, p.207-216, 1992.

PERECIN, D.; BARBOSA, J. C. Comparação entre planos de amostragem seqüencial para presença-ausência e contagens usando dados sobredispersos. Revista de Matemática e Estatística, v.13, p.165-180, 1995.

PEREIRA, M. F. A.; BOIÇA JÚNIOR, A. L.; BARBOSA, J. C. Amostragem seqüencial (presença-ausência) para Bemisia tabaci (Genn.) biótipo B (Hemiptera: Aleyrodidae) em feijoeiro (Phaseolus vulgaris L.). Neotropical Entomology, v.3, n.4, p.499-504, 2004.

PEROSA, J. M. Y.; PIERRE, F. C. Técnicas de pós-colheita e expansão da cultura da manga no Estado de São Paulo. Revista Brasileira de Fruticultura, v.24, n.2, p.381-384, 2002.

PIETERS, E. P.; STERLING, W. L. Sequential sampling cotton squares damaged by boll weevils and Heliothis spp. in the coastal bend of Texas. Journal of Economic Entomology, v.68, n.4, p.543-545, 1975.

RÉGNIÈRE, J.; BOULET, B.; TURGEON, J. J. Sequential sampling plan with two critical levels for spruce bud moth (Lepidoptera: Tortricidae). Journal of Economic Entomo$\log \mathbf{y}$, v.81, n.1, p.220-224, 1988.

SCORZA JUNIOR, R. P.; AQUINO, L. H. DE; BUENO, V. H. P. Plano de amostragem sequencial para avaliação de infestação de Therioaphis trifolli (Monell) f. maculata (Homoptera: Aphididae) em Albany, Califórnia, EUA. Ciência e Agrotecnologia, v.20, n.3, p.346-351, 1996. 
SILVEIRA NETO, S. Monitoramento e decisão no controle de pragas. In: CROCOMO, W.B. (Org.). Manejo Integrado de Pragas. Botucatu: UNESP; São Paulo: CETESB, 1990. p.71-86.

SHELTON, A. M.; NYROP, J. P.; NORTH, R. C.; PETZOLDT, C.; FOSTER, R. Development and use of a dynamic sequential sampling program for onion thrips, Trhips tabaci (Thysanoptera: Thripidae), on onions. Journal of Economic Entomology, v.80, n.5, p.1051-1056, 1987.

SHEPARD, M.; CARNER, G. R. Distribution of insects in soybean field. The Canadian Entomologist, v.108, p.767-771, 1976.

SHIELDS, E. J.; SPECKER, D. R. Sampling for potato leafhopper (Homoptera: Cicadellidae) on alfalfa in New York: relative efficiency of three sampling methods and development of a sequential sampling plan. Journal of Economic Entomology, v.82, n.4, p.1091-1095, 1989.

SILVEIRA NETO, S.; NAKANO, O.; BARBIN, D.; NOVA, N. A. V. Manual de Ecologia dos insetos. São Paulo: Ceres, 1976, 416p.

SOUZA FILHO, M.F.; COSTA, V.A.; PAZINI, W.C. Manejo Integrado de Pragas na cultura da manga. In: ROZANE, D.E.; DAREZZO, R.J.; AGUIAR, R.L.; AGUILLERA, H.A.; ZAMBOLIM, L. (Eds.). Manga: Produção integrada, industrialização e comercialização. Viçosa: UFV, 2004. p.339-376.

STERLING, W. L. Sequential sampling of cotton insect populations. In Beltwide Cotton Prod. Res. Congr., New Orleans: Proccedings, 1975. p.133-135.

TAYLOR, L. R. Assessing and interpreting the spatial distributions of insect populations. Annual Review of Entomology, v.29, p.321-357, 1984.

VIEIRA NETO, J.; AQUINO, L. H.; BEARZOTI, E.; SOUZA, J. C. Otimização da amostragem seqüencial para o monitoramento do bicho-mineiro do cafeeiro Perileucoptera coffeella (Lepidoptera: Lyonetidae) em Lavras, Minas Gerais. Ciência e Agrotecnologia, v.23, n.3, p.707-718, 1999. 
VILLACORTA, A.; TORNERO M. T. T. Plano de amostragem seqüencial de dano causado por Perileucoptera coffeella no Paraná. Pesquisa Agropecuária Brasileira, v.17, n.9, p.1249-1260, 1982.

WALD, A. Sequential tests of statistical hypothesis. Annals of Mathematical Statistics, v.16, p.117-186, 1945.

WALD, A. Sequential Analysis. Nova York: John Wiley, 1947. 212p.

WALKER, P. W.; ALLSOPP, P. G. Sampling distributions and sequential sampling plans for Eumargarodes laingi and Promargarodes spp. (Hemiptera: Margarodidae) in Australian sugarcane. Environmental Entomology, v.22, n.1, p.10-15, 1993.

WILSON, L. T.; ROOM, P. M. Clumping patterns of fruit and arthropods in cotton with implications for binomial sampling. Environmental Entomology, v.12, p.50-58, 1983.

WILSON, L. T.; PICKEL, C.; MOUNT, R. C.; ZALOM, F. G. Presence-abscence sequential sampling for cabbage aphid and green peach aphid (Homoptera: Aphididae) on brussels sprouts. Journal of Economic Entomology, v.76, n.3, p.476-479, 1983.

WRIGHT, L. C.; CONE, W. W.; MENZIES, G. W.; WILDMAN, T. E. Numerical and binomial sequential sampling plans for the hop aphid (Homoptera: Aphididae) on hop leaves. Journal of Economic Entomology, v.83, n.4, p.1388-1394, 1990. 